\title{
The dual effect of viscosity on bubble column hydrodynamics
}

\author{
Giorgio Besagni $^{\mathrm{a}, *}$, Fabio Inzoli ${ }^{\mathrm{a}}$, Giorgia De Guido ${ }^{\mathrm{b}}$, Laura Annamaria Pellegrini ${ }^{\mathrm{b}}$ \\ a Politecnico di Milano, Department of Energy, Via Lambruschini 4a, 20156 Milano, Italy \\ b Politecnico di Milano, Dipartimento di Chimica, Materiali e Ingegneria Chimica "G. Natta", Piazza Leonardo da Vinci 32, 20133 Milano, Italy
}

\section{A R T I C L E I N F O}

\section{Keywords:}

Bubble column

Flow regime transition

Bubble size distributions and shapes

Gas holdup

Viscosity

Aspect ratio correlation

\begin{abstract}
A B S T R A C T
Some authors, in the last decades, have observed the dual effect of viscosity on gas holdup and flow regime transition in small-diameter and small-scale bubble columns. This work concerns the experimental investigation of the dual effect of viscosity on gas holdup and flow regime transition as well as bubble size distributions in a large-diameter and large-scale bubble column. The bubble column is $5.3 \mathrm{~m}$ in height, has an inner diameter of $0.24 \mathrm{~m}$, and can be operated with gas superficial velocities in the range of $0.004-0.20 \mathrm{~m} / \mathrm{s}$. Air was used as the dispersed phase, and various water-monoethylene glycol solutions were employed as the liquid phase. The water-monoethylene glycol solutions that were tested correspond to a viscosity between $0.9 \mathrm{mPa}$ and 7.97 $\mathrm{mPa} \mathrm{s}$, a density between $997.086 \mathrm{~kg} / \mathrm{m}^{3}$ and $1094.801 \mathrm{~kg} / \mathrm{m}^{3}$, a surface tension between $0.0715 \mathrm{~N} / \mathrm{m}$ and $0.0502 \mathrm{~N} / \mathrm{m}$, and $\log _{10}(\mathrm{Mo})$ between -10.77 and -6.55 (where $M o$ is the Morton number). Gas holdup measurements were used to investigate the global fluid dynamics and the flow regime transition between the homogeneous flow regime and the transition flow regime. An image analysis method was used to investigate the bubble size distributions and shapes for different gas superficial velocities, for different solutions of watermonoethylene glycol. In addition, based on the experimental data from the image analysis, a correlation between the bubble equivalent diameter and the bubble aspect ratio was proposed. The dual effect of viscosity, previously verified in smaller bubble columns, was confirmed not only with respect to the gas holdup and flow regime transition, but also for the bubble size distributions. Low viscosities stabilize the homogeneous flow regime and increase the gas holdup, and are characterized by a larger number of small bubbles. Conversely, higher viscosities destabilize the homogeneous flow regime and decrease the gas holdup, and the bubble size distribution moves toward large bubbles. The experimental results suggest that the stabilization/destabilization of the homogeneous regime is related to the changes in the bubble size distributions and a simple approach, based on the lift force, was proposed to explain this relationship. Finally, the experimental results were compared to the dual effect of organic compounds and inorganic compounds: future studies should propose a comprehensive theory to explain all the dual effects observed.
\end{abstract}

\section{Introduction}

\subsection{Bubble column fluid dynamics}

Two-phase bubble columns are equipment used for bringing one or several gases in the form of bubbles (or "coalescence-induced" bubbles) into contact with a liquid phase (either pure or a mixture). Bubble columns are typically built in several forms, but the simplest configuration consists of a vertical cylinder with no internals, in which the gas enters at the bottom through a gas sparger that may vary in design (Deckwer, 1992) (i.e., porous sparger, perforated plates, ring or spider spargers, ...). Eventually, internal devices may be added to control the heat transfer, to limit the liquid phase back-mixing, or to foster the bubble break-up rate. The liquid phase may be supplied in the batch mode or may be led in either co-currently or counter-currently to the upward gas stream. These contacting devices have found many applications in the chemical production industry and biotechnology thanks to their simplicity of construction, the lack of any mechanically operated parts, low energy input requirements (gas dispersion only and liquid recirculation in some cases), reasonable prices and high performance (i.e., a large contact area between the liquid and gas phase and good mixing within the liquid phase throughout the column) (see refs. (Leonard et al., 2015; Rollbusch et al., 2015)). The heat and mass transfer that occurs in these gas-liquid systems does not necessarily involve reactions between gas and liquid phases, even if this occurs in some cases. For example, oxidation, hydrogenation, chlorination, phosgenation, alkylation and other processes are performed in bubble column reactors (Zehner and Kraume, 2005).

\footnotetext{
* Corresponding author

E-mail addresses: giorgio.besagni@polimi.it (G. Besagni), fabio.inzoli@polimi.it (F. Inzoli), giorgia.deguido@polimi.it (G. De Guido), laura.pellegrini@polimi.it (L.A. Pellegrini).
} 


\author{
Nomenclature \\ Non-dimensional numbers \\ $E o=\frac{g\left(\rho_{L}-\rho_{G}\right) d_{e q}^{2}}{\sigma}$ Eötvös number \\ $F r=\frac{U^{2}}{g d_{e q}} \quad$ Froude number \\ $M o=\frac{g\left(\rho_{L}-\rho_{G}\right) \mu_{L}^{4}}{\rho_{L}^{2} \sigma^{3}}$ Morton number \\ $R e=\frac{\rho_{L} U d_{e q}}{\mu_{L}}$ Reynolds number \\ $W e=\frac{d_{e q} U^{2} \rho_{L}}{\sigma}$ Weber number \\ $N_{\mu}={\frac{\mu_{L}}{\mu_{G}}}^{\sigma} \quad$ Viscosity ratio \\ Acronyms \\ $\begin{array}{ll}\text { BSD } & \text { Bubble Size Distribution } \\ \text { CMC } & \text { Carboxymethyl cellulose } \\ \text { MEG } & \text { Monoethylene glycol }\end{array}$
}

Symbols

a Major axis of the bubble [m]

$b \quad$ Minor axis of the bubble [m]

$c_{i}(i=1, \ldots, 5)$ Coefficient in the ellipse equation (Eq. (15))

[Dimensionless]

$c_{M E G} \quad$ Mass concentration of MEG [\%]

$c_{L} \quad$ Lift coefficient in Eq. (27) [Dimensionless]

$d_{23} \quad$ Sauter mean diameter [mm]

$<d_{23}>\quad$ Gas velocity-average mean diameter [mm]

$d_{o} \quad$ Gas sparger holes diameter [mm]

$d_{c} \quad$ Diameter of the column [m]

$d_{e q} \quad$ Bubble equivalent diameter $[\mathrm{mm}]$

$D_{H} \quad$ Hydraulic diameter [m]

$D_{H}^{*} \quad$ Non-dimensional diameter [Dimensionless]

$D_{H, C r}^{*}$ Critical non-dimensional diameter [Dimensionless]

$g \quad$ Acceleration due to gravity $\left[\mathrm{m} / \mathrm{s}^{2}\right]$

$h \quad$ Height along the column [m]

$H_{c} \quad$ Height of the column [m]

$H_{D} \quad$ Height of the free-surface after aeration [m]

$H_{O} \quad$ Height of the free-surface before aeration [m]

$J \quad$ Drift-flux [m/s]

$k_{i}(i=1, \ldots, 5)$ Coefficients in the aspect ratio correlation [Dimensionless] $m \quad$ Exponent in Eq. (19) [Dimensionless]

$n \quad$ Exponent in Eqs. (13) and (14) [Dimensionless]

$N \quad$ Number of classes used in Eq. (26) [Dimensionless]

$p_{i}(i=1,2,3) \quad$ Coefficients in the parabola equation (Eq. (18))

[Dimensionless]

$S_{i}(i=1,2,3)$ Parameters in the swarm velocity method (Eq. (8)) [Dimensionless]

$T \quad$ Temperature [K]

$t_{G} \quad$ Mean residence time of the dispersed phase [s]

$U_{b} \quad$ Parameter in the drift-flux method (Eq. (11)) [m/s]

$U_{\infty} \quad$ Terminal velocity of an isolated bubble (Eqs. (11)-(13)) $[\mathrm{m} / \mathrm{s}]$

$U \quad$ Superficial velocity $[\mathrm{m} / \mathrm{s}]$

$u \quad$ Mean rise velocity $[\mathrm{m} / \mathrm{s}]$

$v_{b} \quad$ Bubble terminal velocity through the Clift diagram $[\mathrm{m} / \mathrm{s}]$

$z_{i}(i=1, \ldots, 5)$ Coefficients in the aspect ratio correlation

$V \quad$ [Dimensionless]

Greek symbols

$\alpha \quad$ Proportionality coefficient in Eq. (19) [Dimensionless]

$\alpha_{G} \quad$ Local gas fraction in Eq. (27) [Dimensionless]

$\beta \quad$ Exponent in Eq. (19) [Dimensionless]

$\gamma \quad$ Volume fraction contribution (Eq. (33)) [Dimensionless]

$\varepsilon \quad$ Holdup [Dimensionless]

$\lambda \quad$ Ratio between $d_{e q}$ and $d_{c}$ [Dimensionless]

$v \quad$ Bubble terminal velocity $[\mathrm{m} / \mathrm{s}]$

$\rho \quad$ Density $\left[\mathrm{kg} / \mathrm{m}^{3}\right]$

$\sigma \quad$ Surface tension $[\mathrm{N} / \mathrm{m}]$

$\phi \quad$ Aspect ratio [Dimensionless]

Subscripts

c Parameter related to the bubble column

Slug-bubble Parameter related to slug-bubbles

$L \quad$ Liquid phase

$G \quad$ Gas phase

$T, E \quad$ Subscripts in the drift-flux formulation (Eqs. (10)-(12))

trans Transition point

swarm Swarm velocity

wallis Wallis plot method
Despite the simple column arrangement, bubble columns are characterized by extremely complex fluid dynamic interactions and coupling between the phases. For this reason, their correct design, operation and scale-up rely on the knowledge of the fluid dynamics at different scales: mainly, the "bubble-scale" (i.e., bubble size distributions and shapes, single bubble dynamics, collective bubble dynamics, ...) and the "reactors-scale" (i.e., flow patterns, mean residence time of the disperse phase, dynamics of mesoscale clusters, ...). At the "bubblescale", the bubble motion and bubble dynamics characterize and influence the medium-scale circulation (i.e., eddies that transport the dispersed phase) and the large-scale circulation (i.e., the liquid phase flowing upward in the center of the column and downward in the region near the wall). A typical approach is to apply scale-up methods to estimate the fluid dynamics of industrial-scale reactors from laboratory-scale facilities. Subsequently, models for the interfacial heat and mass transfer and, eventually, to take into account the multi-phase reactions, are applied. The knowledge of the fluid dynamics at the different scales can be quantified through the precise estimation of the local (i.e., the bubble size distributions, BSD, and the bubble aspect ratio; "bubble-scale") and the global (i.e., the gas holdup, $\varepsilon_{G}-\mathrm{a}$ dimensionless parameter defined as the volume of the gas phase divided by the total volume; "reactor-scale") fluid dynamic properties. Indeed, at the "bubble-scale", the size and shape of the interface of the dispersed phase characterize the heat and mass exchange; conversely, at the "reactor-scale", the gas holdup determines the residence time and, in combination with the BSD, the interfacial area for the rate of interfacial mass transfer (related to the reactor size). The global and local fluid dynamic properties are strictly related to the prevailing flow regime: the homogeneous flow regime, the transition flow regime and the heterogeneous flow regime (if considering large-diameter bubble columns, see refs. (Nedeltchev, 2015; Nedeltchev and Schubert, 2015; Nedeltchev and Shaikh, 2013)). In the following, these flow regimes are described: (i) first, the general definitions are given; (ii) secondly, the literature concerning the flow regimes is summarized; (ii) finally, the definition of the flow regimes within this research is given.

The homogeneous flow regime is, generally, associated with small gas superficial velocities $\left(U_{G}\right)$ and is characterized by limited interactions between the bubbles. The transition flow regime is characterized by large flow macro-structures with large eddies and a widened bubble size distribution due to the onset of bubble coalescence. The hetero- 
geneous flow regime is associated with high gas superficial velocities, high coalescence and breakage phenomena and a wide variety of bubble sizes. In large-diameter bubble columns, the slug flow regime does not exist: this concept has been listed in the scale-up criteria proposed by Wilkinson et al. (Wilkinson et al., 1992) and has been previously noted a review by Shah et al. (Shah et al., 1982). In particular, Shah et al. (Shah et al., 1982) have cited the work of Hills (Hills, 1976), who in turn cited Ellis and Lloyd Jones (Ellis and Lloyd Jones, 1965) ("Ellis noted a similar result that slugs could not be seen in wider tubes, although large bubbles or bubble clusters were undoubtedly present" (Hills, 1976)). These observations rely on the fluid dynamics phenomena and the coupling between the gas and liquid phases, which can be interpreted considering the Rayleigh-Taylor instabilities, as proposed by Kitscha and Kocamustafaogullari (Kitscha and Kocamustafaogullari, 1989). The quantification of the RayleighTaylor instabilities on the "reactor-scale" is quantified through the dimensionless diameter, $D^{*}{ }_{H}$, which is related to the Eötvös number of the slug bubbles as follows:

$$
\begin{aligned}
D_{H}^{*} & =\frac{D_{H}}{\sqrt{\sigma / g\left(\rho_{L}-\rho_{G}\right)}} \stackrel{\text { Circular bubble column }}{\Longrightarrow} \frac{d_{c}}{\sqrt{\sigma / g\left(\rho_{L}-\rho_{G}\right)}} \\
& =\frac{1}{\sqrt{\sigma / d_{c}^{2} g\left(\rho_{L}-\rho_{G}\right)}}=\frac{1}{\sqrt{1 / E o_{c}}}=\sqrt{E o_{c}}=\sqrt{E o_{\text {slug-bubble }}}
\end{aligned}
$$

In Eq. (1), $D_{\boldsymbol{H}}$ is the hydraulic diameter, $d_{\boldsymbol{c}}$ is the bubble column (inner) diameter, $\sigma$ is the surface tension, $g$ is the acceleration due to gravity, $\rho_{L}-\rho_{G}$ is the density difference between the two phases, and $E o_{c}=E o_{\text {slug-bubble }}$ is the Eötvös number computed using the bubble column diameter, which is also the characteristic length of the slug bubbles. Bubble columns with $D^{*}{ }_{H}$ greater than the critical value $D_{H, \boldsymbol{c r}}^{*}=52$ (see ref. (Brooks et al., 2012))-equivalent to $E o_{\text {slug- }}$ bubble $=7.21$ (i.e., $d_{c} \gtrsim 0.13-0.15 \mathrm{~m}$; ambient temperature and pressure)-are considered to be large-diameter bubble columns. When the bubble column diameter is larger than $D^{*}{ }_{H}, \boldsymbol{c r}$, changes in the fluid dynamics appear: the cap bubbles can no more be sustained, and "coalescence-induced" bubbles (or, cluster of bubbles) appear instead of the slug flow regime. Consequently, the fluid dynamic properties in large-diameter bubble columns differ from the flow in small-diameter columns. For this reason the flow regime maps, flow regime transition criteria and correlations used to predict the twophase flow in small-diameter bubble columns may not be scaled up to predict the flow in large-diameter columns.

Despite the fact that the classification reported above for the homogeneous, transition and heterogeneous flow regimes is widely used and accepted, it is over simplified. Indeed, the interactions between the phases inside the bubble columns are extremely complex, making the classification of the flow regimes not straightforward. In practice, both small and large bubbles may appear at low $U_{G}$ values (Besagni and Inzoli, 2016a, 2016b): for example, when a sparger with large openings is used, the quality of the gas distribution is poor, large bubbles are aerated and a "gas maldistribution flow regime" (as defined by Nedetlchev (Nedeltchev et al., 2016; Nedeltchev and Schubert, 2015)) is established at low $U_{G}$ values. Kaji et al. (Kaji et al., 2001) defined, as homogeneous flow regime, the flow regime in which discrete bubbles are generated from a sparger and are dispersed uniformly without coalescence. In contrast, Wilkinson et al. (Wilkinson et al., 1992) defined a homogeneous flow regime as the flow regime in which the gas holdup increases linearly with increasing $U_{G}$, regardless of the uniformity of the BSD. Kazakis et al. (Kazakis et al., 2007) defined the flow regime in which large and small bubbles coexist in a laminar flow as "pseudo-homogeneous flow regime". Yang et al. (Yang et al., 2010) distinguished between the homogeneous and the "pseudohomogeneous" flow regimes: the former denotes the flow regime characterized by a uniform BSD, whereas the latter indicates the flow regime in which discrete bubbles are generated from the sparger and the gas holdup increases almost linearly with increasing $U_{G}$, but no uniformity in the radial bubble distribution near the sparger region exists. For the sake of clarity, an accurate definition of the homogeneous flow regime within this research is needed, and is proposed in the following.

In this work, the homogeneous flow regime is referred to as the flow regime where only "non-coalescence-induced" bubbles exist (as detected by the gas disengagement technique, see ref. (Besagni and Inzoli, 2016b)). The homogeneous flow regime is then classified as a "purehomogeneous" (or "mono-dispersed homogeneous") flow regime and a "pseudo-homogeneous" (or "poly-dispersed homogeneous" or "gas maldistribution") flow regime: the former is characterized by a mono-dispersed BSD, whereas the latter is characterized by a polydispersed BSD. The mono-dispersed and poly-dispersed BSDs are defined, in our research, according to the change in the sign of the lift force coefficient (Besagni et al., 2016b; Besagni and Inzoli, 2016b; Lucas et al., 2015; Zahradnik et al., 1997; Ziegenhein et al., 2015). The transition flow regime is identified by the appearance of the "coalescence-induced" bubbles (Besagni and Inzoli, 2016b) and is characterized by large flow macro-structures with large eddies and a widened bubble size distribution due to the onset of bubble coalescence. At high gas velocities, a fully heterogeneous flow regime is reached (Sharaf et al., 2015); it is associated with high coalescence and breakage rates and a wide variety of bubble sizes. The transitions between the different flow regimes depend on the operation mode, the design parameters of the bubble column and the properties of the gas/liquid phases. For example, using a "fine sparger" $\left(d_{O}<1 \mathrm{~mm}\right.$, such as for porous spargers, membranes, needles, sieve tray/perforated plates with small openings) the homogeneous flow regime is stabilized (Mudde et al., 2009) and a "pure-homogeneous" flow regime exists, which leads to the hindrance effect that physically is manifested by the peak on the gas holdup graph. In contrast, using a "coarse sparger" $\left(d_{O}>1 \mathrm{~mm}\right)$ the "pure-homogeneous" flow regime may not exist and a "pseudo-homogeneous" flow regime is observed at lower gas superficial velocities; finally, when using a "very coarse sparger" $\left(d_{O}>>1 \mathrm{~mm}\right)$, the homogeneous flow regime may not be established and a "pure heterogeneous flow regime" takes place (Ruzicka et al., 2001).

The many relationships between the bubble column fluid dynamic parameters and the various variables characterizing the system (i.e., the column design, the operation modes and the properties of the phases) make it difficult to find general correlations for the precise design of the bubble columns (i.e., the correct estimation of the gas holdup, the bubble size distributions and shapes). In this respect, our research activity aims in providing a complete set of experimental data to develop a general correlation to estimate the "reactor-scale" parameters (i.e., the gas holdup), taking into account the "bubblescale" (i.e., bubble size distributions and shapes) and, thus, to provide a rational basis for the design and scale-up of bubble column reactors. To this end, we have set up a large-scale experimental facility $(5.3 \mathrm{~m}$ in height and inner diameter of $0.24 \mathrm{~m}$ ) and we have addressed three main lines of research. The experimental setup was built considering the scale-up criteria proposed by Wilkinson et al. (Wilkinson et al., 1992), after performing experiments in two different column diameters $\left(d_{c}=0.15\right.$ and $\left.0.23 \mathrm{~m}\right)$, at different operating pressures and using different liquid phases (n-heptane, monoethylene glycol, and water). Based on their own results as well as on literature data, they have concluded that the gas holdup is independent of the column dimensions and the sparger design if the following criteria are satisfied:

1. criterion 1. The diameter of the bubble column, $d_{c}$, is larger than $0.15 \mathrm{~m}$;

2. criterion 2. The aspect ratio, $A R$ (the ratio between the height and the diameter of the column), is larger than 5 ;

3. criterion 3. The sparger openings diameter, $d_{o}$, is larger than $1-$ $2 \mathrm{~mm}$ ("coarse" spargers).

In our research, three main lines of research have been pursued. 
First, experimental methods to estimate the bubble size and shapes have been proposed (Besagni et al., 2016a; Besagni and Inzoli, 2016a). Secondly, the influence of internals (Besagni and Inzoli, 2016b, 2016c), of the operation modes (Besagni et al., 2014, 2016b; Besagni and Inzoli, 2016b, 2016c) over the bubble column fluid dynamics has been studied. Thirdly, the influence of the liquid phase properties has been investigated and, in particular, we have focused our attention on the two kinds of surface active compounds: (i) organic substances-i.e., ethanol (Besagni et al., 2016b)-and (ii) inorganic substances-i.e., sodium chloride (Besagni and Inzoli, 2015). In our experimental investigations, gas holdup measurements have been used to investigate the flow regime transition, and the image analysis and optical probes have been used to provide insight in the homogeneous flow regime (i.e., refer to the comprehensive experimental procedure has been detailed in our previous paper, (Besagni and Inzoli, 2016b)).

This paper further discusses the influence of the liquid phase properties at the "bubble-scale" and at the "reactor scale" by focusing on the effect of viscous solutions over gas holdup, flow regime transition and BSDs. In the following, a literature survey on gas holdup, flow regime transition, and BSDs is presented; for every study considered, the details on the column geometry (i.e., column diameter, $d_{c}$ ), aspect ratio (free liquid, $H_{o}$, and/or column height, $H_{c}$ ) and sparger openings $\left(d_{o}\right)$ are given since they must be provided to obtain a correct comparison of the different experimental investigations, according the criteria discussed by Besagni and Inzoli (Besagni and Inzoli, 2016b). Finally, given the literature survey, the main goals of this paper are stated.

\subsection{Influence of viscosity on the gas holdup}

In the previous studies, both the increase and the decrease of the gas holdup were observed as the liquid viscosity, $\mu_{L}$, , is increased. For example, Yoshida and Akita (Akita and Yoshida, 1973; Yoshida and Akita, 1965) $\left(d_{c}=0.152 \mathrm{~m}\right)$ reported that the gas holdup varies with the liquid viscosity in an irregular manner. Other studies reported a decrease of the gas holdup while increasing the liquid viscosity. Wilkinson et al. (Wilkinson et al., 1992) $\left(d_{c}=0.158 \mathrm{~m}, H_{O}=1.5 \mathrm{~m}\right.$, ring sparger, $d_{O}=2 \mathrm{~mm}-d_{c}=0.15-0.23 \mathrm{~m}, H_{O}=1.2 \mathrm{~m}$, ring sparger, $d_{o}=7 \mathrm{~mm}$ ) found that a high viscous liquid phase (monoethylene glycol, $\mu_{L}=21 \mathrm{mPa} \cdot \mathrm{s}$ ) causes a decrease in the gas holdup. Kuncová and Zahradnik (Kuncová and Zahradník, 1995) $\left(d_{c}=0.15 \mathrm{~m}, H_{c}=1 \mathrm{~m}\right.$, $H_{\boldsymbol{O}}=0.5 \mathrm{~m}$, perforated plate, $d_{\boldsymbol{O}}=0.5 \mathrm{~mm}$ ) investigated the effect of liquid viscosity on the dynamics of bubble bed formation using several aqueous solutions of saccharose $\left(1<\mu_{L}<110 \mathrm{mPa} \cdot \mathrm{s}\right)$ : they found a decrease in the gas holdup while increasing the viscosity. Hwang and Cheng (Hwang and Cheng, 1997) $\left(d_{c}=0.19 \mathrm{~m}, H_{c}=2.5 \mathrm{~m}\right.$, ring sparger, $d_{O}=1 \mathrm{~mm}$ ) investigated the gas holdup structure in highly viscous Newtonian and non-Newtonian media using water and Carboxymethyl cellulose (CMC) solutions: they observed that the gas holdup decreases when the viscosity increases. Zahradnik et al. (Zahradnik et al., 1997) $\left(d_{c}=0.15 \mathrm{~m}, H_{O}=0.53 \mathrm{~m}\right.$, perforated plate, $d_{O}=0.5 \mathrm{~mm}$ ) found that moderate/high viscosities (aqueous saccharose solutions, $3<\mu_{L}<110 \mathrm{mPa} \cdot \mathrm{s}$ ) decrease the gas holdup. Yang et al. (2010) $\left(d_{c}=0.15 \mathrm{~m}, H_{c}=1.7 \mathrm{~m}\right.$, perforated plate, $\left.d_{o}=0.7 \mathrm{~mm}\right)$ investigated the influence of the viscosity $\left(1<\mu_{L}<31.5 \mathrm{mPa}\right.$ ) on the gas holdup by using a viscosity-increasing agent (acrylates/C10C30 alkyl acrylate cross-polymer): they observed that the gas holdup decreases when the viscosity increases. Other studies suggested a dual effect of the viscosity (which is the starting point of this research). The pioneering study of Eissa and Schügerl (1975) $\left(d_{c}=0.12 \mathrm{~m}, H_{c}=3.9 \mathrm{~m}\right.$, perforated plate, $d_{O}=2 \mathrm{~mm}$ ) showed that the gas holdup first increases $\left(\mu_{L}<3 \mathrm{mPa} \mathrm{s}\right)$, then decreases $\left(3<\mu_{L}<11 \mathrm{mPas}\right)$, and finally becomes roughly constant $\left(\mu_{L}>11 \mathrm{mPa}\right.$ ). Bach and Pilhofer (Bach and Pilhofer, 1978) $\left(d_{c}=0.10 \mathrm{~m}\right.$, perforated plate, $d_{\boldsymbol{O}}=0.5 \mathrm{~mm}$, as reported by Godbole et al. (Godbole et al., 1982)) observed a similar behavior (as reported by Wilkinson et al. (Wilkinson et al., 1992) for
$U_{G}=0.03 \mathrm{~m} / \mathrm{s}$ and $\left.U_{G}=0.05 \mathrm{~m} / \mathrm{s}\right)$ : the gas holdup first increases $\left(\mu_{L}\right.$ $<1.5-2 \mathrm{mPa} \cdot \mathrm{s})$, then decreases $\left(3<\mu_{L}<11-12 \mathrm{mPa} \cdot \mathrm{s}\right)$, and finally becomes roughly constant $\left(\mu_{L}>12 \mathrm{mPa}\right.$ s). Godbole et al. (1982) ( $d_{c}$ $=0.305 \mathrm{~m}, H_{c}=2.44 \mathrm{~m}$, perforated plate, $d_{O}=1.66 \mathrm{~mm}$ ) studied the gas holdup structure in highly viscous Newtonian and non-Newtonian media using glycerin and CMC solutions: they observed a maximum for glycerin solutions at approximately $\mu_{L}=2 \mathrm{mPa} \cdot \mathrm{s}$. In addition, they observed that, for CMC solutions, the gas holdup first increases up to, approximately, $\mu_{L}=2.23-4.75 \mathrm{mPa}$ s, then it decreases $\left(7.81<\mu_{L}<\right.$ $52.29 \mathrm{mPa}$ s, depending on $U_{G}$ ), and finally becomes roughly constant $\left(\mu_{L}>52.29 \mathrm{mPa} \cdot \mathrm{s}\right)$. Khare and Joshi (Khare and Joshi, 1990) $\left(d_{c}\right.$ $=0.20 \mathrm{~m}, H_{c}=3.0 \mathrm{~m}$, sieve plate, $d_{O}=2.0 \mathrm{~mm}$ ) studied the influence of viscosity on the gas holdup using aqueous solutions of glucose: as a result, they observed that the gas holdup first increases up to $\mu_{L}=4$ $\mathrm{mPa} \mathrm{s}$ and then it decreases for $4<\mu_{L}<10 \mathrm{mPa} \cdot \mathrm{s}$. Ruzicka et al. (Ruzicka et al., 2003) $\left(d_{c}=0.14 \mathrm{~m}, H_{O}=0.2-0.8 \mathrm{~m}\right.$, perforated plate, $d_{O}=0.5 \mathrm{~mm}$ ) summarized the influence of the viscosity over the gas holdup as follows: with increasing liquid viscosity the gas holdup increases for $\mu<3 \mathrm{mPa} \cdot \mathrm{s}$ and it decreases for $3<\mu<22 \mathrm{mPa}$. Olivieri et al. (2011) $\left(d_{c}=0.12 \mathrm{~m}, H_{c}=2 \mathrm{~m}, H_{O}=0.8 \mathrm{~m}\right.$, needle sparger, $d_{O}$ $=0.4 \mathrm{~mm}$ ) investigated the effect of the liquid viscosity using several aqueous solutions of Alginate within the viscosity range 1-117 $\mathrm{mPa}$ s: they reported an increase in the gas holdup up to $\mu_{L}=4.25 \mathrm{mPa} \mathrm{s}$ and, then, a decrease at higher viscosities.

These apparently contradictory results can be explained by interpreting them in terms of the "dual effect of viscosity over the gas holdup". At low viscosity, the coalescence is limited and the large drag force reduces the bubble rise velocity, causing an increase in the gas holdup. When increasing the viscosity, the tendency to coalescence prevails, creating large bubbles rising the column at a higher velocity, thus reducing the gas holdup. In particular, the "dual effect of the viscosity" was clearly observed in some experimental investigations (Bach and Pilhofer, 1978; Eissa and Schügerl, 1975; Godbole et al., 1982; Khare and Joshi, 1990; Olivieri et al., 2011; Rabha et al., 2014; Ruzicka et al., 2003), but it is not clear whether it also applies to larger bubble columns (see, for example, the fluid dynamics phenomena behind Eq. (1)) and what the reasons are behind this behavior. At present, Godbole et al. (1982) and Khare and Joshi (1990) are the only investigators who studied the dual effect in a large-diameter bubble column, but they only focused on the gas holdup.

\subsection{Influence of viscosity on the flow regime transition}

It is well known that the shape of the gas holdup curve (the relationship between the gas holdup and the gas superficial velocity) mainly depends on the gas sparger used and the diameter of its holes (Sharaf et al., 2015; Urseanu, 2000). "Coarse spargers" leads to monotonic gas holdup curves, whereas, when using "fine spargers", a peak in the gas holdup curve can appear (owing to the hindrance effects of the mono-dispersed BSDs). It is interesting that, at higher viscosities, even for fine spargers, the peak in the gas holdup curve can disappear (Kuncová and Zahradník, 1995; Olivieri et al., 2011; Zahradnik et al., 1997). This behavior may be explained by the changes in the hydrodynamics and the prevailing flow regimes in the bubble column: for the sake of clarity, an overview of the main effects of the viscosity in the homogeneous flow regime is presented hereafter.

In air-water bubble columns-operating at ambient temperature and pressure-the homogeneous flow regime ends at approximately $U_{G}$, trans $\approx 0.04 \mathrm{~m} / \mathrm{s}$ (Deckwer and Field, 1992). Depending on the many variables characterizing the system, $U_{G}$, trans either reduces ("homogeneous flow regime destabilization") or increases ("homogeneous flow regime stabilization”). The liquid phase viscosity, depending on its value, may either stabilize or destabilize the homogeneous flow regime and this may be interpreted as the "dual effect of viscosity over the flow regime transition". The destabilization of the homogeneous flow regime is caused by the increased coalescence as 
discussed and experimentally observed by Wikinson et al. (Wilkinson et al., 1992) and, more recently, by Yang et al. (Yang et al., 2010). The increased coalescence may also suppress the homogeneous flow regime and, for $\mu_{L}>8 \mathrm{mPa} \cdot \mathrm{s}$, it may not exist even with a 'fine sparger' (Kuncová and Zahradník, 1995; Ruzicka et al., 2003; Zahradnik et al., 1997). Other authors discussed the effect of liquid viscosity on the flow regime transition, indicating that many basic questions about this effect remain unanswered. Ruzicka et al. (2003) encountered this effect when using aqueous solutions of glycol. They found out that the homogeneous flow regime is stabilized at low viscosities $\left(\mu_{L}=1-3\right.$ $\mathrm{mPa} \mathrm{s}$ ) and that $U_{G}$, trans increases with the viscosity in this range, whereas it is destabilized at moderate viscosities $\left(\mu_{L}=3-22 \mathrm{mPa} \mathrm{s}\right)$. Olivieri et al. (2011) reported a stabilization of the homogeneous flow regime up to $\mu_{L}=4.25 \mathrm{mPa} \mathrm{s}$ and, then, for higher viscosities, a destabilization of the homogeneous flow regime. These phenomena were also confirmed by the ultrafast electron bean X-ray tomography investigation of Rabha et al. (Rabha et al., 2014) $\left(d_{c}=0.07 \mathrm{~m}, H_{c}\right.$ $=1.5 \mathrm{~m}$, orifice sparger, $d_{O}=1 \mathrm{~mm}$ ): beyond $\mu_{L}=5.18-8.95 \mathrm{mPa} \mathrm{s}$, the homogeneous was no longer detected.

\subsection{Influence of viscosity on the bubble size distributions and shapes}

To the authors' opinion, the relation between the homogeneous flow regime stabilization/destabilization and the gas holdup increase/ decrease is strictly related to the BSD characterizing the system. This concept is thoroughly discussed in the framework of this research. High viscous media are characterized by a stable bubble interface and thereby promote the formation of large bubbles at the sparger (Deckwer, 1992), bubble coalescence (Deckwer, 1992; Kuncová and Zahradník, 1995; Wilkinson et al., 1992; Zahradnik et al., 1997) and decreases of the bubble breakup rate (Shah et al., 1982; Wilkinson et al., 1992). The presence of large bubbles advances the flow regime transition. In highly viscous media, a bimodal population, in terms of the bubble equivalent diameter ( $d_{\text {eq }}$, Section 2.3.2), is observed: for example, $d_{e q}<1 \mathrm{~mm}$ and $d_{e q}>20 \mathrm{~mm}$ (Philip et al., 1990), $1 \mathrm{~mm}<$ $d_{e q}<10 \mathrm{~mm}$ and $10<d_{e q}<150 \mathrm{~mm}$ (Godbole et al., 1982), $0.7 \mathrm{~mm}$ $<d_{e q}<10 \mathrm{~mm}$ and $d_{e q}>10 \mathrm{~mm}$ (Yang et al., 2010). Rahba et al. (Rabha et al., 2014) reported bimodal bubble size distributions with large bubbles having $d_{e q}=40-45 \mathrm{~mm}$. For example, for viscosities

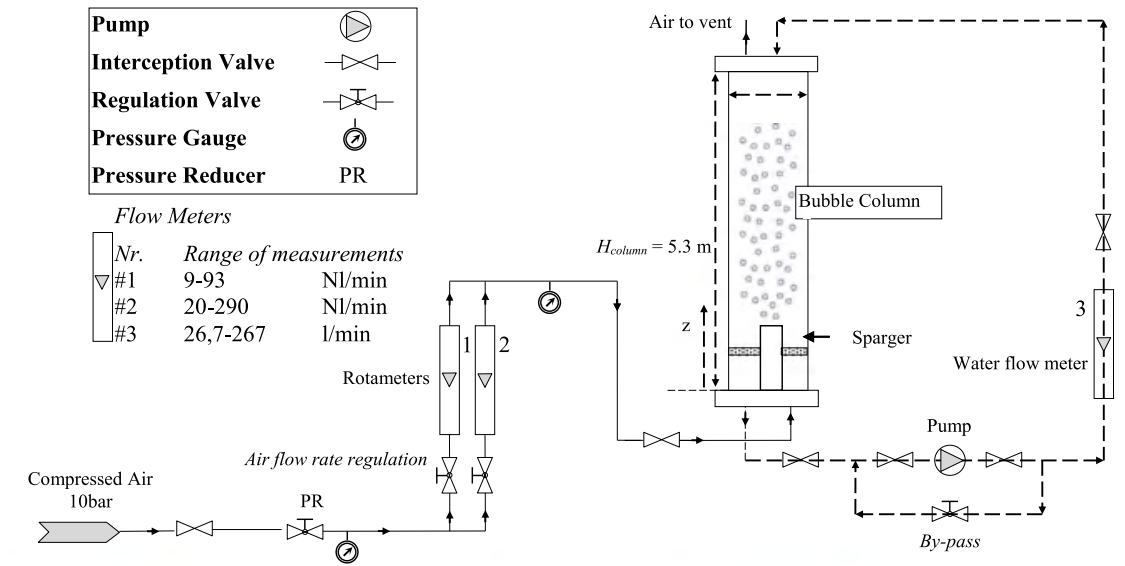

(a) Experimental facility

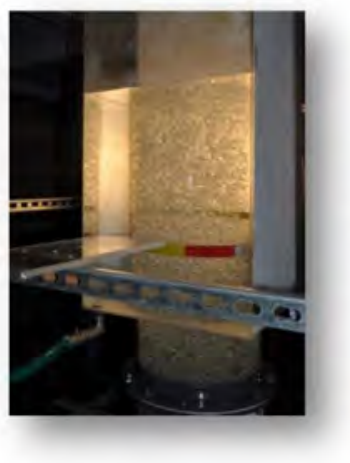

(b) Box for flow visualization

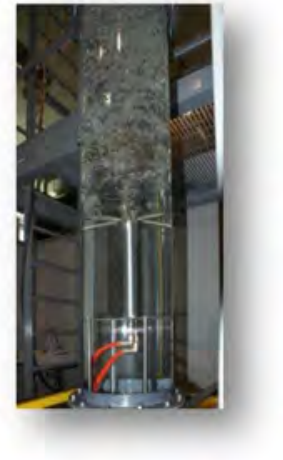

(c) Sparger

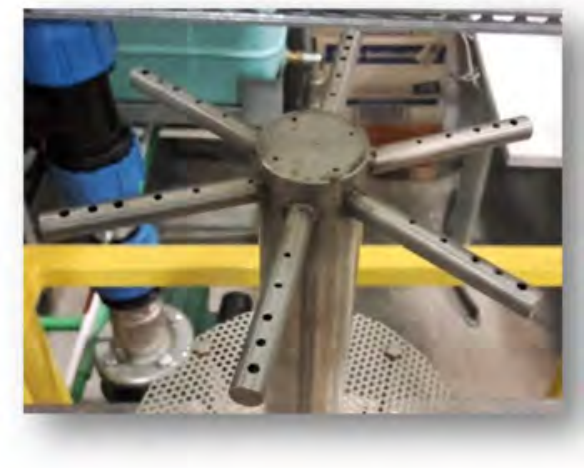

(d) Spider sparger: view from top

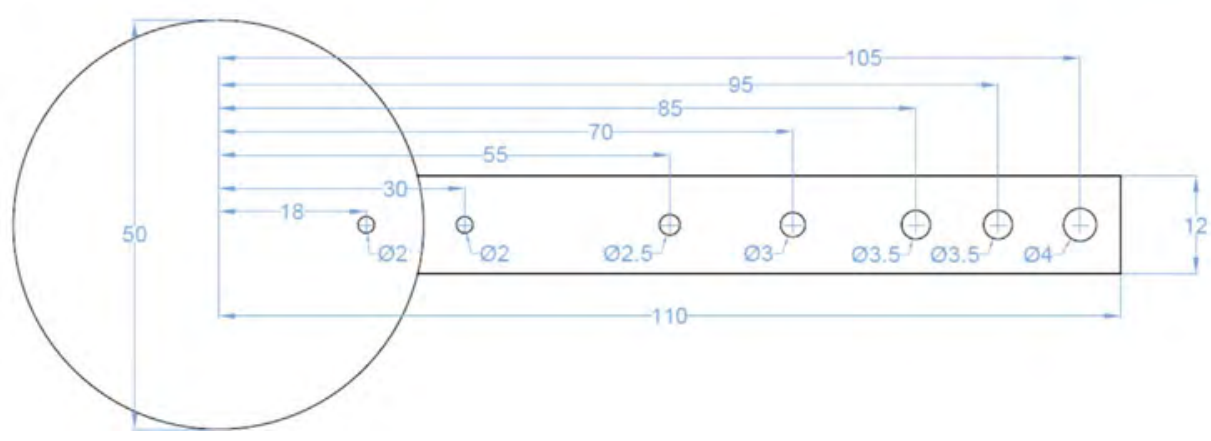

(e) Spider sparger: distribution of the holes on one of the six arms (measurements in $\mathrm{mm}$ )

Fig. 1. Experimental setup and measurement techniques. 
beyond $\mu_{L}>30 \mathrm{mPa}-\mathrm{s}$, the contribution of the small bubbles results in a further increase of the total gas holdup with increasing viscosity (Kuncová and Zahradník, 1995), instead of the levelling at a constant value, as reported by Eissa and Schügerl (1975). An exception with the above references is the study of La Rubia et al. (2010), who observed a decrease of $d_{e q}$ from 4.6 to $4.2 \mathrm{~mm}$ while increasing $U_{G}$. At present, it is not clear whether a "dual effect of viscosity over BSD" exists.

Beside the BSD, the bubble shapes should be considered (i.e., the aspect ratio of the bubbles, which is the ratio of the maximum vertical dimension to the maximum horizontal dimension). Indeed, the interface shape and size is important to characterize multiphase flows properly. The significance of the bubble shape is clear if we consider the heat and mass transfer at the interface and the closures in computational fluid dynamics. For example, (i) the drag force models needs to contain the information about the bubble shape; (ii) the lift force seems to be influenced by the aspect ratio (Tomiyama et al., 2002). Summarizing, the aspect ratio is a basic parameter for modeling and understanding bubbly flows. In this perspective, there is a lack of research analyzing bubble shapes (especially when concerning swarms of bubbles), and proposing novel correlations between the bubble size and shapes. Some attempts were made to correlate the aspect ratio as a function of dimensionless parameters (see refs. (Celata et al., 2004; Celata et al., 2006; Celata et al., 2007)). Some authors have used the Eötvös number, Eo (Okawa et al., 2003; Wellek et al., 1966), others adopted the Weber number, We (Moore, 1959; Taylor and Acrivos, 1964; Wellek et al., 1966), while Tadaki and Maeda (Tadaki and Maeda, 1961) used the Tadaki number (Clift et al., 1978). Other authors used more than one dimensional number, i.e. Bozzano and Dente used both the $M o$ and the Eo numbers (Bozzano and Dente, 2001) and Aoyama et al. (Aoyama et al., 2016) used the Re and the Eo numbers. However, these correlations were developed for single bubbles/drops, and they may not be suitable for dense bubbly flows. This issue was discussed, at first, by Besagni and Inzoli (Besagni and Inzoli, 2016a) and, later, by Besagni et al. (Besagni et al., 2016a) who analyzed bubble sizes and shapes in air-water bubble column and proposed correlations for the aspect ratio valid for dense bubbly flows. Despite the fact that some studies investigated the shapes of single rising bubbles in viscous media (Aoyama et al., 2016; Dimakopoulos et al., 2013; Fraggedakis et al., 2016; Gumulya et al., 2016; Sikorski et al., 2009; Tsamopoulos et al., 2008), the relations between the bubble size and shapes in dense bubbly flows with viscous media remains unanswered. In addition, the available databases of the bubble aspect ratios in viscous liquids are still insufficient, as observed by Aoyama et al. (2016), and additional data are needed to better establish and extend the range of validity of the existing correlations to estimate the bubble shapes.

\subsection{Scope of the paper}

Despite the fact that the effect of viscosity on bubble column hydrodynamics is well assessed in small-diameter and small-scale bubble columns, there is a lack of research concerning large-diameter columns (except for a few studies that have been stated above, which focus only on this effect on the gas holdup). Indeed, the effects of the viscous solutions, reported in small-diameter bubble columns, may not be used for large-diameter bubble columns (Eq. (1)). Furthermore, there is a lack of research that provides insight into the dual effect by relating the gas holdup, the flow regime transition, and the BSDs.

Our experimental facility and investigation discuss the existing gap in the previous literature. The present bubble column has an inner diameter of $d_{c}=0.24 \mathrm{~m}$ (thus, it is a large diameter-pipe - Eq. (1), $D^{*}{ }_{H}$ =88.13) and a height of $H_{c}=5.3 \mathrm{~m}$ (aspect ratio $H_{c} / d_{c}>20$ ). The diameter of the column, its height and the sparger openings were chosen considering the well-known scale up criteria proposed by Wilkinson et al. (1992) (stated above).

Taking into account the literature survey and the current state-of- the-art, this study has four main goals: .

1. to verify the dual effect of the viscosity on the gas holdup, and flow regime transition and to study the influence of the viscosity on the BSDs (it is not clear whether the dual effect of viscosity may apply also to BSD or not) in a large-diameter and large-scale bubble column (whose results may be used for scale-up purposes);

2. to investigate the relations between the "bubble-scale" (i.e., BSDs and bubble shapes) and the "reactor-scale" (i.e., gas holdup, flow regime transition) in order to provide an insight in the dual effect of viscosity and in the effect of the system surface tension;

3. to study the influence of viscosity on bubble size distributions and shapes and to propose a correlation for the bubble aspect ratio in dense bubbly flows;

4. to provide a comprehensive experimental dataset for model development and validation.

To this end, in this study, various solutions of water-monoethylene glycol of different concentrations have been employed as the liquid phase, corresponding to viscosity between $0.9 \mathrm{mPa}-\mathrm{s}$ (tap water) and $7.97 \mathrm{mPa}-\mathrm{s}$, density between $997.086 \mathrm{~kg} / \mathrm{m}^{3}$ and $1094.801 \mathrm{~kg} / \mathrm{m}^{3}$, surface tension between $0.0715 \mathrm{~N} / \mathrm{m}$ and $0.0502 \mathrm{~N} / \mathrm{m}$, and $\log (\mathrm{Mo})$ between -10.77 and -6.55 (where, Mo is the Morton number). The experimental investigation has consisted in gas holdup measurements and image analysis. The gas holdup measurements have been used for investigating the global hydrodynamics and for analyzing the flow regime transition. The image analysis technique-aimed to study the bubble size distributions and shapes-has been used for different gas superficial velocities (in the homogeneous flow regime) for different concentrations. To the authors' best knowledge, this is the first study concerning the dual effect of viscosity on gas holdup, flow regime transition and BSDs in a large-diameter bubble columns.

This paper is structured as follows. In Section 2, the experimental setup is presented and the measurement techniques are detailed. In Section 3, the experimental results are presented and the relation between the bubble size distributions and the flow regime transition is discussed. Finally, the main conclusions are given.

\section{Experimental setup and method}

\subsection{Experimental setup and liquid phases}

The experimental facility (Fig. 1a) is a non-pressurized vertical pipe made of Plexiglas ${ }^{\circledast}$ with $d_{c}=0.24 \mathrm{~m}$ (Eq. (1), $D^{*}{ }_{\boldsymbol{H}}=88.13$ ) and $H_{\boldsymbol{c}}$ $=5.3 \mathrm{~m}$. A pressure reducer controls the pressure upstream from the rotameters (1) and (2), used to measure the gas flow rate (accuracy \pm $2 \%$ f.s.v., E5-2600/h, manufactured by ASA, Italy). The experimental facility has been also equipped with boxes for flow visualization (Fig. 1b, Section 2.3). The values of gas density (used to compute the gas superficial velocity) are based upon the operating conditions

Table 1

Physical properties for the MEG concentrations investigated (computed at 1 bar and $298.15 \mathrm{~K})$.

\begin{tabular}{lllll}
\hline $\mathbf{c}_{\mathbf{M E G}}[\mathbf{w t} \mathbf{0}]$ & $\boldsymbol{\rho}_{\mathbf{L}}\left[\mathbf{k g} / \mathbf{m}^{\mathbf{3}}\right]$ & $\boldsymbol{\mu}_{\mathbf{L}}[\mathbf{m P a} \cdot \mathbf{s}]$ & $\boldsymbol{\sigma}[\mathbf{N} / \mathbf{m}]$ & $\log _{\mathbf{1 o}}(\mathbf{M o})[-]$ \\
\hline 0 & 997.086 & 0.8903 & 0.0715 & -10.7724 \\
0.05 & 997.158 & 0.8917 & 0.0715 & -10.7697 \\
0.1 & 997.229 & 0.8928 & 0.0715 & -10.7676 \\
0.5 & 997.801 & 0.9019 & 0.0713 & -10.7466 \\
0.75 & 998.159 & 0.9077 & 0.0712 & -10.7337 \\
1 & 998.516 & 0.9135 & 0.0711 & -10.721 \\
5 & 1004.208 & 1.0106 & 0.0696 & -10.5202 \\
8 & 1008.443 & 1.0894 & 0.0685 & -10.3708 \\
10 & 1011.249 & 1.145 & 0.0677 & -10.2703 \\
40 & 1051.15 & 2.4287 & 0.0583 & -8.78602 \\
80 & 1094.801 & 7.9655 & 0.0502 & -6.54542 \\
\hline
\end{tabular}


existing at the column mid-point (computed by using the ideal gas law) (Reilly et al., 1994). The gas distributor, is a spider-sparger distributor (Fig. 1c, d) with hole diameters $d_{o}=2-4 \mathrm{~mm}$ (Fig. 1e). The spider sparger, shown in Fig. 1c and d, has six arms made of $0.12 \mathrm{~m}$ diameter stainless steel tubes soldered to the center cylinder of the sparger. The sparger has been installed with the six holes located on the side of each arm facing upward; these holes are distributed as shown in Fig. 1e, with an increasing diameter moving toward the column wall.

The gas and liquid temperatures have been checked and maintained constant at room temperature during all the experiments ( $295 \pm 1 \mathrm{~K})$. Filtered air from laboratory lines has been used as the gaseous phase in all the experiments; the air-cleaning line consists in filters (mechanical and activated carbon) and condensation drying unit, in order to clean the gas phase properly and, thus, to avoid the presence of contaminants in form of (i) solid particles and (ii) organic substances. The liquid phase has included different solutions of deionized water and monoethylene glycol (MEG). In such a case, a liquid of a known composition has been charged to the column and the gas flow rate adjusted to the desired value. Considering ambient temperature and pressure conditions, the properties for pure water have been taken from refs. (Kestin et al., 1978; Perry and Green, 2008; Vargaftik et al., 1983). As for the physical properties of water-MEG mixtures, the work by Sun and Teja (2003) has been taken into account for evaluating their densities and viscosities as a function of the mass fraction of monoethylene glycol, of the temperature and of the properties of the two components constituting the binary system. As for the surface tension of aqueous monoethylene glycol solutions at $T=298.15 \mathrm{~K}$, the correlation found in the product guide provided by MEGlobal ${ }^{\mathrm{TM}}$ (MEGlobalTM, 2015) for monoethylene glycol aqueous solutions has been used:

$\sigma[m N / m]=71.536-0.39671 \cdot\left(100 \cdot c_{M E G}\right)+0.001625 \cdot\left(100 \cdot c_{M E G}\right)^{2}$

Table 1 summarizes the monoethylene glycol aqueous solutions studied and their properties (density, viscosity and surface tension), and the corresponding Mo number, computed using the above equations (the reader may refer to the nomenclature section for the definitions of non-dimensional numbers).

During the experimentation, great care has been taken to ensure that the bubble column has been always clean to minimize any contamination that might affect the results (the system has been previously flushed to remove contaminants and to avoid the presence of additional surfactants). A discussion concerning the influence of the liquid phase and salt quality was discussed by Rucizka et al. (Ruzicka et al., 2008) and Orvalho et al. (2009).

\subsection{Gas holdup measurements and flow regime transition analysis}

\subsubsection{Gas holdup measurements}

Measurements of the bed expansion allowed the evaluation of the gas holdup, $\varepsilon_{G}$ ("reactor-scale" experimental data). The procedure involves measuring the location (height) of the liquid free surface when air flows in the column. The gas holdup is then obtained using the relation:

$\varepsilon_{G}=\frac{V_{G}}{V_{L+G}} \stackrel{\text { Constant cross-section-area }}{\Longrightarrow} \frac{\left(H_{D}-H_{0}\right)}{H_{D}}$

where $H_{D}$ and $H_{O}$ are the heights of the free-surface after and before aeration, respectively $\left(H_{O}=3.0 \mathrm{~m}\right.$ above the distributor, $\left.H_{O} / d_{c}=12.5\right)$. Further studies will be devoted to understand the influence of the liquid level on the bubble column hydrodynamics. The gas holdup curve (the relation between $\varepsilon_{G}-U_{G}$ ) provides information of the global bubble column fluid dynamics and can be used to study the flow regime transitions (secondary data, as described in Section 2.2.2). Indeed, applying the mass conservation to the gas phase, the gas holdup is computed as:

$\varepsilon_{G}=U_{G} / U_{\text {swarm }} \rightarrow U_{\text {swarm }}=U_{G} / \varepsilon_{G} \sim t_{G}$
Where $U_{\text {Swarms }}$ is the mean rise velocity of the gas phase (it can be computed by the experimental measurements obtained through Eq. (4)) and $t_{G}$ is the mean residence time of the gas phase. $U_{\text {Swarms }}$ is strictly related to the coupling between the phases and the main parameters (i.e., bubble sizes, rise velocities, ...) and, thus, to the mean residence time of the gas phase, $t_{G}$ (Orvalho et al., 2009; Ruzicka et al., 2008).

\subsubsection{Flow regime transition}

Two main transitions exist in large-diameter bubble columns (as stated in the introduction):

(i) the transition between the homogeneous flow regime and the transition flow regime (in the present bubble column, the "monodispersed homogeneous" does not exist (as observed in our previous study, (Besagni and Inzoli, 2016b) and the "pseudohomogeneous regime" is observed);

(ii) the transition between the transition flow regime and the heterogeneous flow regime.

Although the flow regimes transitions do not occur instantaneously, the definition of approximate transition points is helpful to understand and model the fluid dynamic behavior of bubble columns (Krishna et al., 1991). Many authors consider only the first flow regime transition, without any reference to the second one, except for a limited number of studies (Nedeltchev, 2015; Nedeltchev and Shaikh, 2013; Sharaf et al., 2015). In the following, for the sake of clarity, we refer to "flow regime transition point" considering the first flow regime transition. In our previous paper we have showed how the swarm velocity and the drift-flux/Wallis plot methods have been able to identify the first flow regime transition point (Besagni and Inzoli, 2016b). In particular, the first flow regime transition point, in terms of the transition gas velocity and transition gas holdup, is computed as the average of the values obtained by the two methods:

$$
\begin{aligned}
U_{G, \text { trans }} & =\frac{U_{G, \text { trans,swarm }}+U_{G, \text { trans,Wallis }}}{2} \\
\varepsilon_{G, \text { trans }} & =\frac{\varepsilon_{G, \text { trans,swarm }}+\varepsilon_{G, \text { trans,Wallis }}}{2}
\end{aligned}
$$

The details concerning the two methods are provided below. It is worth noting that the transition point between the transition and the heterogeneous flow regime cannot be identified by means of these two methods, which is a matter of future studies.

2.2.2.1. Swarm velocity method. The swarm velocity method has been developed by Zuber and Findlay (1965) and is based on the swarm velocity (as defined in Eq. (4)). The swarm velocity is plotted against the gas superficial velocity: $U_{\text {swarm }}$ is almost constant in the homogeneous flow regime (in some cases, it can be slightly decreasing), but it starts to increase as the system enters the heterogeneous flow regime at a transition superficial velocity, $U_{G}$, trans. The appearance of the first "coalescence-induced" bubble is responsible for this sudden increase in the swarm velocity and is an indication of flow regime transition. This method has previously been employed by Krishna et al. (1991), Letzel et al. (1997), Gourich et al. (2006), Ribeiro Jr and Mewes (2007) and Besagni et al. (Besagni et al., 2014, 2016b; Besagni and Inzoli, 2015, 2016a, 2016c). In this study, the quantitative evaluation of $U_{G}$, trans has been determined by the intersection between the trends of $U_{\text {swarm }}$ in the two flow regimes.

Thus, $U_{\text {swarm }}$ has been taken as constant in the homogeneous flow regime (Eq. (7)):

$U_{\text {swarm, } \text {, }}$ omogeneous $=$ constant

whereas, in the transition flow regime it has been determined by a least 
squares fitting of the following function:

$U_{\text {swarm,transition }}=S_{1}\left(U_{G}\right)^{S_{2}}+S_{3}$

where $S_{1}, S_{2}$ and $S_{3}$ are fitting parameters.

The transitional velocity is then evaluated by solving the following equation:

$U_{\text {swarm, } \text { homogeneous }}=U_{\text {swarm, } \text { transition }}$

2.2.2.2. Drift-flux method. The drift-flux method has been proposed by Wallis (1969) and has been widely applied (see, for example, refs. (Passos et al., 2015; Ribeiro Jr and Mewes, 2007; Wallis, 1969)). This method is based on the drift-flux, which represents the gas flux through a surface moving with the speed of the two-phase mixture and is experimentally obtained as follows:

$J_{T}=U_{G}\left(1-\varepsilon_{G}\right) \pm U_{L} \varepsilon_{G}$

In the batch mode, $U_{L}=0 \mathrm{~m} / \mathrm{s}$. The detailed derivation of Eq. (10) has been proposed byBesagni and Inzoli (2016c).

Theoretically, the drift-flux is written in terms of a characteristic bubble velocity, whose dependence upon $\varepsilon_{G}$ varies with the prevailing flow regime:

$J_{E}=U_{b}\left(1-\varepsilon_{G}\right)$

The idea in this method is to employ a model for $U_{b}$ that is valid for the homogeneous flow regime, plot $J_{E}$ and $J_{T}$ in the same graph as a function of $\varepsilon_{G}$. In the homogeneous flow regime, $J_{E}$ is equal to $J_{T}$ and, then, the transition point is defined when:

$J_{T} \neq J_{E}$

The evaluation of $U_{\boldsymbol{b}}$ is a matter of discussion and different models have been proposed and applied. In this study, the approach of Krishna et al. (2000) has been followed, which is based on the empirical model of Richardson and Zaki (1997):

$U_{b}=u_{\infty} \varepsilon_{G}\left(1-\varepsilon_{G}\right)^{n-1}$

where $n$ is fluid-dependent and $u_{\infty}$ is the terminal velocity of an isolated bubble. These values should be fitted with the aid of the experimental data in the determination of the transition point. Combining Eq. (11) and Eq. (13), the following equation is derived:

$J_{E}=u_{\infty} \varepsilon_{G}\left(1-\varepsilon_{G}\right)^{n}$

\subsection{Photography}

\subsubsection{Instrumentation}

The photos have been taken using a NIKON D5000 camera (f/3.5, $1 / 1600$ s, ISO400, $4288 \times 2848$ pixels). The back light method has been employed in the experiments using a $500 \mathrm{~W}$ halogen lamp as light source. Visualization sections consist in squared boxes (filled with water) around the vertical pipe designed for correcting the distorted image (Fig. 1b) (Besagni, 2016; Besagni and Inzoli, 2016a). The camera has been accurately aligned horizontally to the visualization sections. Images have been acquired near the sparger and at, approximately, $h$ $=2.4 \mathrm{~m}$ from the sparger; the region at $h=2.4 \mathrm{~m}$ corresponds to the developed region of the two-phase flow, as demonstrated elsewhere (Besagni and Inzoli, 2016b).

\subsubsection{Image analysis methods}

The goal of the image analysis has been to obtain bubble shape, bubble orientation and bubble size distributions ("bubble-scale" experimental data). The image analysis method considers two kinds of bubbles: (i) spherical/ellipsoidal and (ii) cap-shaped bubbles.
2.3.2.1. Image analysis method for spherical/ellipsoidal bubbles. Ellingsen and Risso (2001) stated that the bubble shape is entirely defined by the knowledge of its major axis and minor axis: under these conditions, the projected contours are ellipses. Considering their conclusion, in this paper, the non cap-shaped bubbles are approximated and reconstructed ellipses. It is worth noting that in the present case, the images were acquired in the developed region of the two-phase flow, where the break-up and coalescence phenomena are not relevant, the bubbles rose mostly rectilinearly, and their shapes were axisymmetric at most (Besagni and Inzoli, 2016b). To sample the spherical/ellipsoidal bubbles, the same approach that was applied to an annular gap bubble column (Besagni et al., 2016b; Besagni and Inzoli, 2016a) and other systems (Besagni and Inzoli, 2016b; Besagni et al., 2016c) has been also used in this work. This approach was already detailed elsewhere (Besagni and Inzoli, 2016a) and is briefly summarized in the following. In this approach, each bubble is approximated and reconstructed using an ellipse, represented by the following equation:

$c_{1} x^{2}+c_{2} x y+c_{3} y^{2}+c_{4} y+c_{5} x+1=0$

The method is structured in three phases:

1. Calibration. The reference conversion factor between pixels and millimeters is provided;

2. Bubble reconstruction. For each bubble, six points on the bubble are selected (by human vision), and ellipse equation parameters $c_{1}$, $c_{2}, c_{3}, c_{4}$ and $c_{5}$ are evaluated using the least squares method;

3. Bubble processing. The equivalent ellipse is processed to obtain the major axis $2 a$, the minor axis $2 b$, the center of the bubble and the orientation angle. Finally, the bubble equivalent diameters, $d_{e q}$, and aspect ratio $\varphi$ are obtained as:

$d_{e q}=2 \sqrt[3]{a^{2} b}$

$\Phi=\frac{b}{a}$

2.3.2.2. Image analysis method for cap-shaped bubbles. The method is structured in three phases:

- Calibration. The reference conversion factor (pixels/mm) is provided;

- Bubble reconstruction. For each bubble, four points on the upper edge of the bubble are selected (by human vision), and ellipse equation parameters $\left(p_{1}, p_{2}\right.$, and $\left.p_{3}\right)$ are computed using the least squares method. The cap-bubbles are approximated using a parabola, represented by:

$y=p_{1} x^{2}+p_{2} x+p_{3}$

The lower region of the bubble is taken into account by a line, connecting the vertex of the above segment.

- Bubble processing. The equivalent parabola is processed to obtain the dimension of the base and of the vertical axis. Then, $d_{e q}$ is obtained equating the volume of the paraboloid to the one of a sphere having the same diameter, $d_{e q}$.

\subsubsection{Bubble sampling}

The number of bubbles to be sampled to achieve a reliable BSD is a matter of discussion (Honkanen et al., 2005). Various studies have sampled different numbers of bubbles-between 50 and 100 (Lage and Espósito, 1999), 200 (Wongsuchoto et al., 2003), 250 (Rakoczy and Masiuk, 2009), 300 (Hanselmann and Windhab, 1998), between 250 

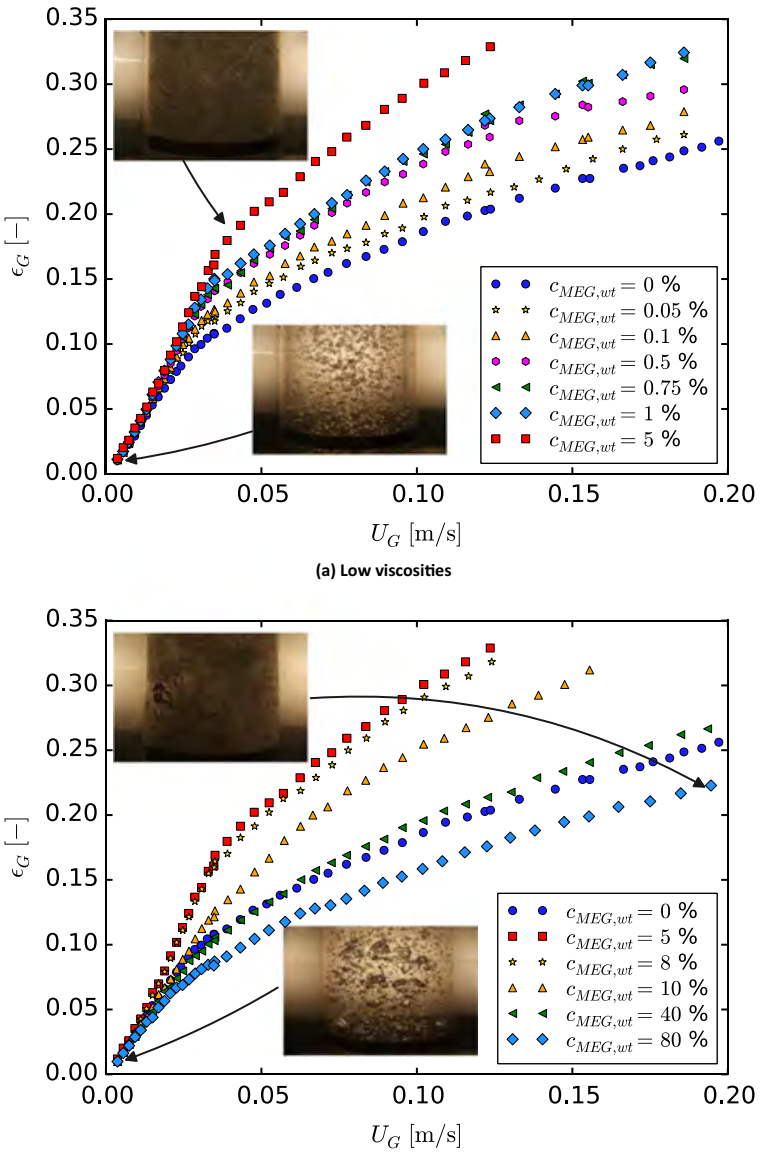

(b) Moderate/High viscosities

Fig. 2. Holdup measurements: influence of MEG concentrations.

and 300 (Aloufi, 2011) and 100 (Passos et al., 2015). The image analysis has been used for five gas superficial velocities and for the different solutions tested. In this study, for each case, at least 800 bubbles have been selected using 2 (or more) photos. A sensitivity analysis concerning the number of bubbles to be sampled has been performed and has been presented in our previous paper (Besagni and Inzoli, 2016a). A ruler along the external wall has been used for the $\mathrm{pixel} / \mathrm{mm}$ conversion to evaluate the size of the bubbles and to avoid references inside the column, which would affect the two-phase flow.

\subsubsection{Image analysis: uncertainties}

The main issues and uncertainties concerning the image analysis are summarized in the following:

- Image distortion. Even if the test section has been covered by the squared box filled with water, some refraction problems may still arise, as the refraction index of pipe wall is different from that of water. Indeed, the refractive index of the Plexiglas ${ }^{\circledR}$ is 1.48 and the one of water is 1.33. This effect is negligible, as demonstrated in the annular gap bubble column configuration (Besagni and Inzoli, 2016a). The same has been verified in the present configuration, by evaluating the conversion factor for a ruler placed in the center of the column for different radial positions (Besagni, 2016). The effect of the optical distortion has been found to be negligible: the maximum difference in the conversion factors is 0.5 pixel.

- Overlapped images. The number of sampling points requested has been larger than the minimum number of points for defining an ellipse: this has allowed sampling the bubbles also in overlapping conditions. However, when increasing $U_{G}$, the bubble overlapping and the reduced back light has been such that there may be a lack of information: it has been verified that the image analysis is suitable for analyzing gas holdups up to $\varepsilon_{G}=0.05$ (Besagni, 2016), regardless of the operation mode and working fluids.

- Projected images. Only projected bubble images could be obtained. This problem is well known and, despite some proposals (Bian et al., 2013; Sahagian and Proussevitch, 1998), a solution is far from being reached and the use of $2 \mathrm{D}$ projected images is a common way of processing bubble images (Karn et al., 2015; Lage and Espósito, 1999; Lau et al., 2013a, 2013b; Wongsuchoto et al., 2003). However, the bubble shape data obtained by this approach are considered reliable: the bubble shape information allowed the extrapolation of correlations for the aspect ratio (Besagni, 2016; Besagni and Inzoli, 2016a) and flow regime transition criteria (Besagni and Inzoli, 2016a). The results of these analyses have been validated with the experimental data and, therefore, the bubble shape information is considered reliable. As for the approximation of bubbles with ellipses, Lage and Espósito (Lage and Espósito, 1999) have stated that the error in the measurement of each axis of the ellipse is approximately 6\%. Taking into account the error introduced by the hypothesis of oblate spheroid and the optical distortion, they have estimated that the experimental error in the determination of $d_{e q}$ is between $10 \%$ and $15 \%$.

- Handpicked points. The analysis concerning the errors made during the bubble shape estimation due to the handpicked points can be found in (Besagni and Inzoli, 2016b).

For further details the reader should refer to the dissertation of Besagni (Besagni, 2016).

\section{The experimental results}

In this section, the influence of the MEG concentration over the bubble column hydrodynamics is presented and discussed. First, the gas holdup curves (the "reactor-scale" experimental data) are presented. Second, the gas holdup curves are analyzed by using statistical methods (refer to Section 2.3) to obtain the flow regime transition points. Finally, the "bubble-scale" data (the BSDs) are presented and discussed.

The detailed analysis of the air-water system (i.e., gas holdup, gas disengagement, flow regime transition, optical probe measurements, and the comparison with previous studies) were discussed elsewhere (Besagni and Inzoli, 2016b).

\subsection{Gas holdup - "reactor-scale"}

\subsubsection{Experimental measurements}

Fig. 2a and b display the gas holdup curves for the different solutions of water-MEG, whereas Fig. 3, Figs. 4 and 5 propose flow visualizations of the two-phase flow. Fig. 3 compares the flow regimes for the air-water and for two air-water-MEG cases $\left(c_{M E G}=5 \%\right.$ and $c_{M E G}=80 \%$ ) Fig. 4 displays the influence of the MEG concentration on bubble shapes in the pseudo-homogeneous flow regime $\left(U_{G}\right.$ $=0.0037 \mathrm{~m} / \mathrm{s}$ ): the poly-dispersed nature of the homogeneous flow regime is clearly visible in Fig. 4 and is quantified in Section 3.3 (thus, the homogeneous flow regime is classified as a pseudo-homogeneous flow regime). Fig. 5 displays the influence of the MEG concentration on the macro-flow structures in the transition flow regime.

All gas holdup curves are similar in shape: at low $U_{G}$-in the pseudo-homogeneous flow regime-the relationship between the $\varepsilon_{G}$ and $U_{G}$ is linear, followed by a change in slope due to the flow regime transition. In this region, the slope changes continuously, indicating a continuous increase of the contribution of large bubbles to the gas holdup (Besagni and Inzoli, 2016b; Urseanu, 2000; Yang et al., 2010). Above the transition velocity, large deformed bubbles begin to appear and the bubble coalescence increases the average rise velocity and reduces gas residence time in the column, hence decreasing the gas 

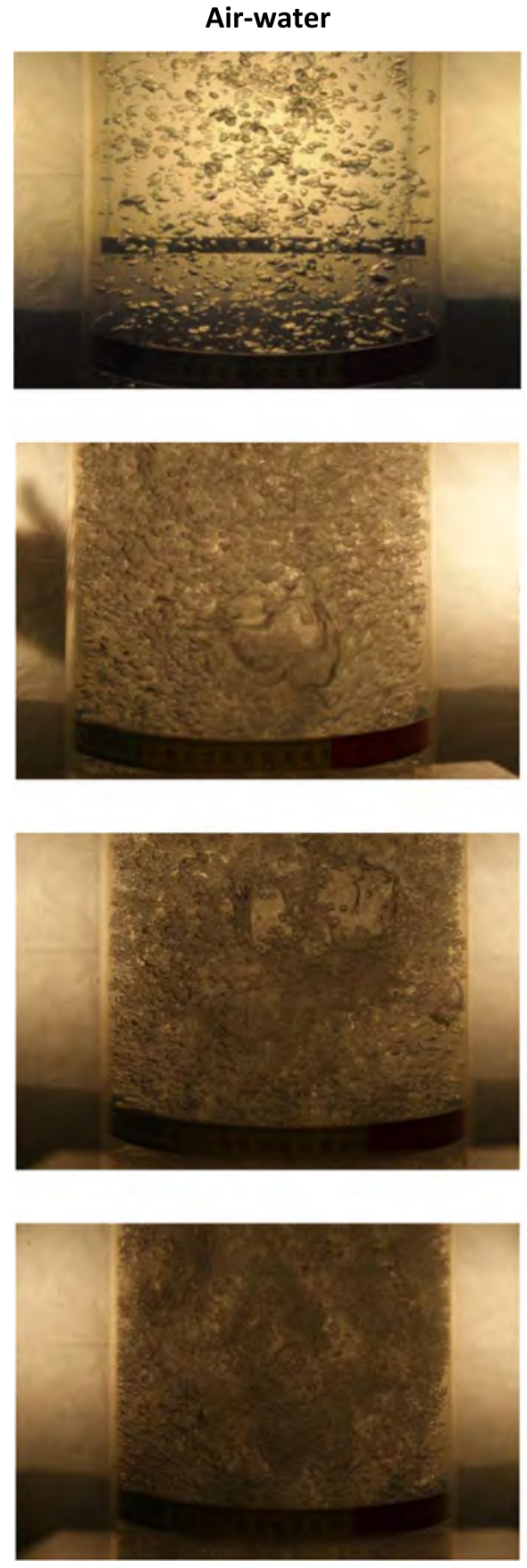

Air-water-MEG 5\% ${ }_{\mathrm{wt}}$

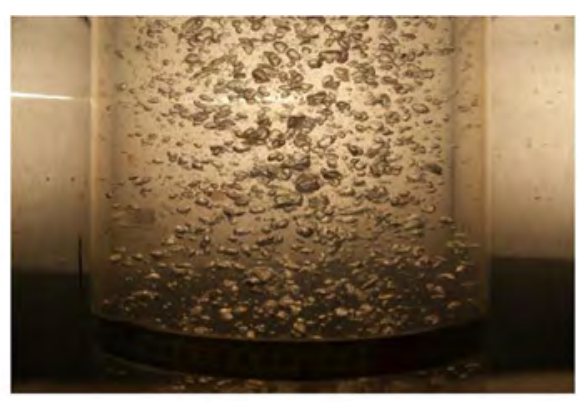

(a) $U_{G}=0.0037 \mathrm{~m} / \mathrm{s}$

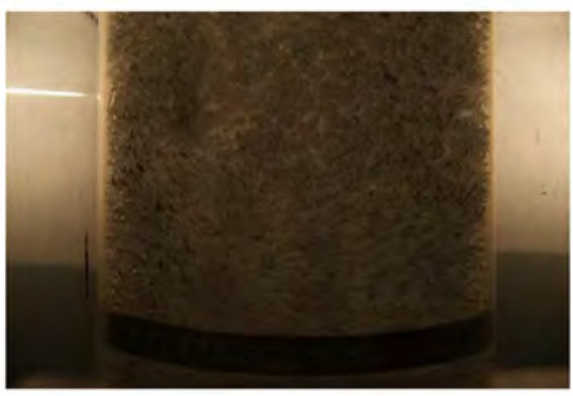

(b) $U_{G}=0.0389 \mathrm{~m} / \mathrm{s}$

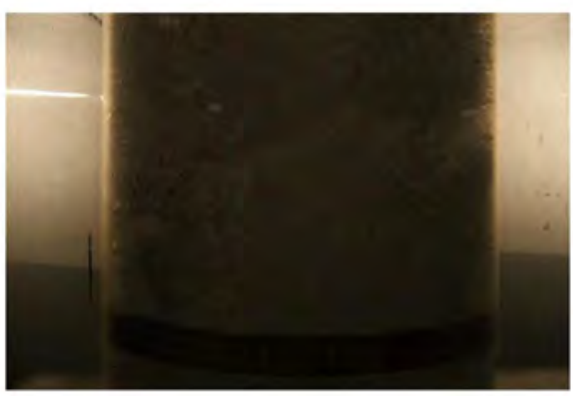

(c) $U_{G}=0.0894 \mathrm{~m} / \mathrm{s}$

\section{Not available}

(Maximum holudp reached, Figure 2a)

\section{Air-water-MEG $80 \%_{\mathrm{wt}}$}
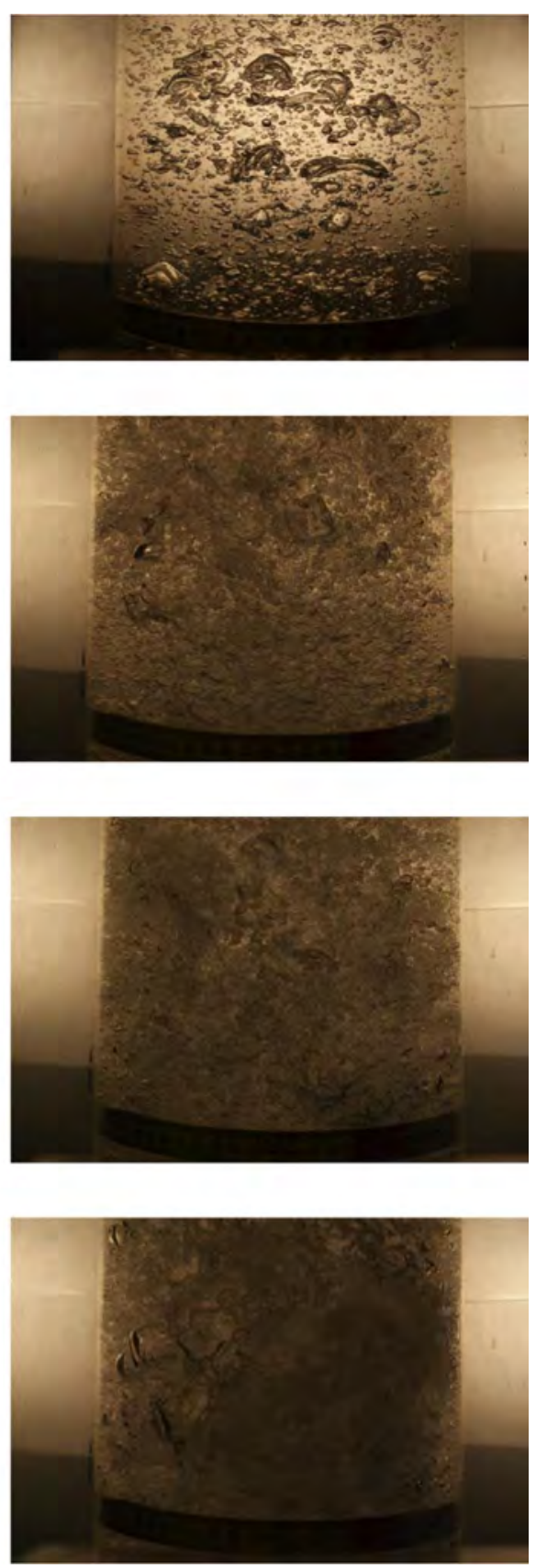

(d) $U_{G}=0.1665 \mathrm{~m} / \mathrm{s}$

Fig. 3. Flow visualizations: influence of MEG concentrations.

holdup versus gas velocity slope (Besagni and Inzoli, 2016b; Yang et al., 2010). The reader should refer to the discussion of Besagni and Inzoli (2016b) for further details on the structure of the gas holdup curve and for a comparison to the previous literature, as far as the air-water case is concerned. Generally, the relationship between $\varepsilon_{G}$ and $U_{G}$ is described by the proportionality:

$\varepsilon_{G}=\alpha \cdot U_{G}{ }^{\beta}$

The values of $\beta$ and $\alpha$ depend on the flow regime; they were already determined for the air-water case and can be found in De Guido et al. (2016), along with the expression of the blending function used to link the two curves (Eq. (20)), with the exponent $m$ being an adaptive parameter to be determined by the minimization of the sum of the absolute deviations between the calculated and experimental gas holdup values.

$\varepsilon_{G, \text { evaluated }}=\left(\varepsilon_{G, \text { homogeneous }}^{-m}+\varepsilon_{G, \text { transition }}^{-m}\right)^{-1 / m}$

The form of the blending function in Eq. (19) was proposed by Churchill and Usagi (1972) for the correlation of rates of transfer and other phenomena. The value of the exponent $m$, which allows for a satisfactory link, the two curves are ultimately $m=9.91$. Future studies will be devoted to extending Eq. (20) to the other air-water-MEG systems. The values of the $\beta$ exponent $(\beta=1.15$ in the homogeneous flow regime and $\beta=0.49$ in the transition flow regime (De Guido et al., 2016)) in Eq. (19) are in agreement with the expectations: when applying mass conservation to the gas phase, the gas holdup is given by $\varepsilon_{G}=U_{G} / u_{G}$, where $u_{G}$ is the mean rise velocity of the gas phase. Theoretically, if the bubbles travel at their terminal velocity, the gas 


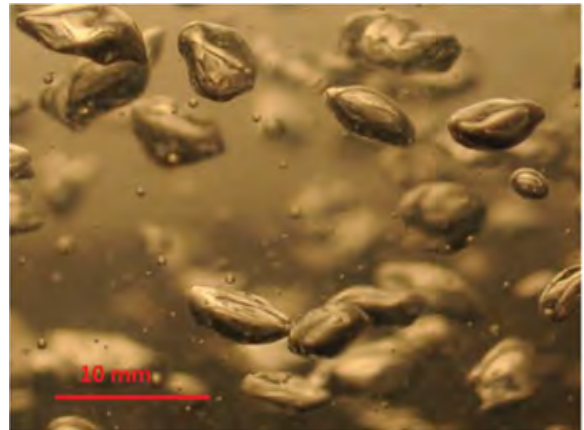

(a) $c_{M E G}=0 \%-\log _{10}(M o)=-10.7724$

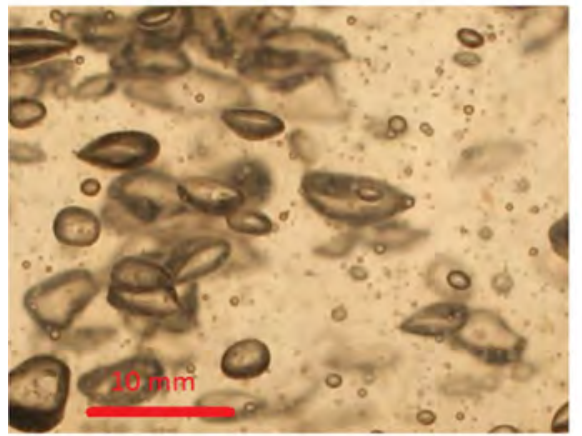

(d) $c_{M E G}=0.5 \%-\log _{10}(M o)=-10.7466$

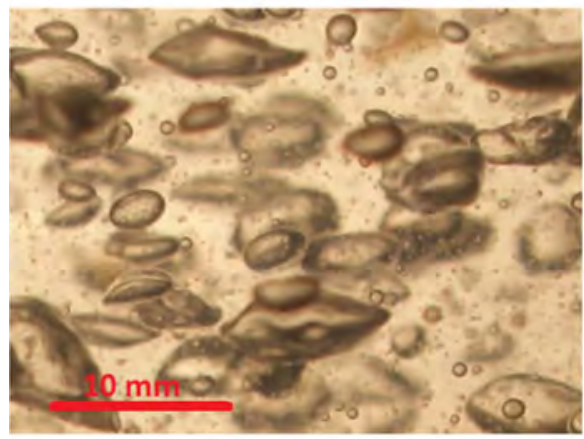

(g) $c_{M E G}=5 \%-\log _{10}(M o)=-10.5202$

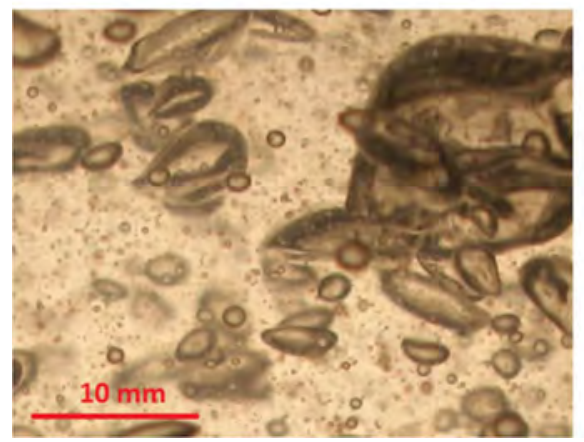

(j) $c_{M E G}=40 \%-\log _{10}(M o)=-8.78602 *$

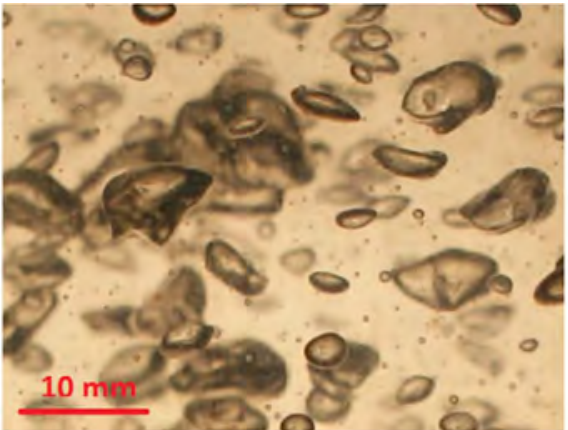

(b) $c_{M E G}=0.05 \%-\log _{10}(M o)=-10.7697$

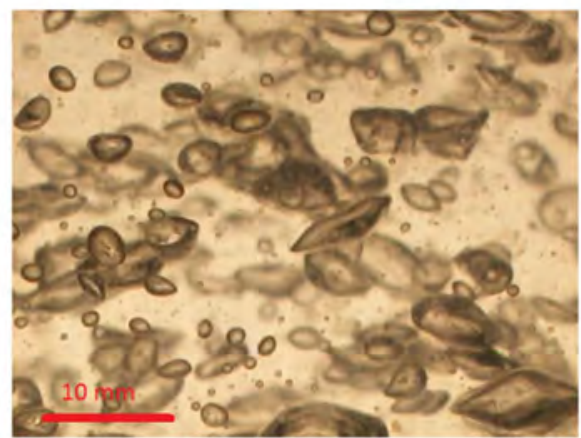

(e) $c_{M E G}=0.75 \%-\log _{10}(M o)=-10.7337$

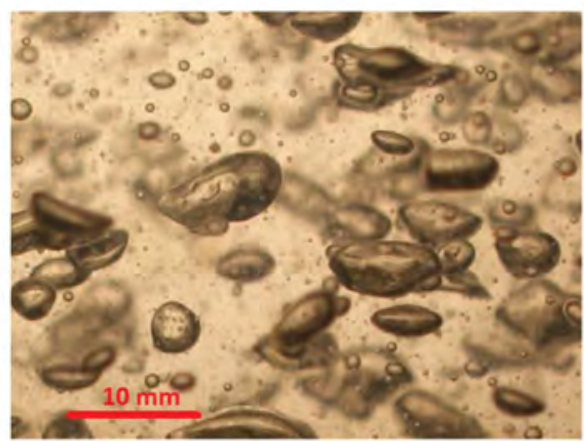

(h) $c_{M E G}=8 \%-\log _{10}(M o)=-10.3708$

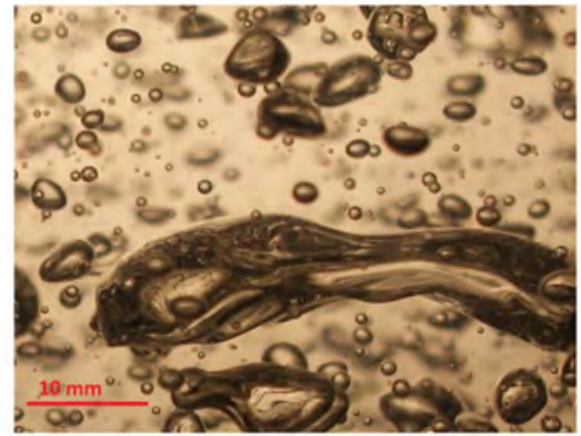

(k) $c_{M E G}=80 \%-\log _{10}(M o)=-6.54542$

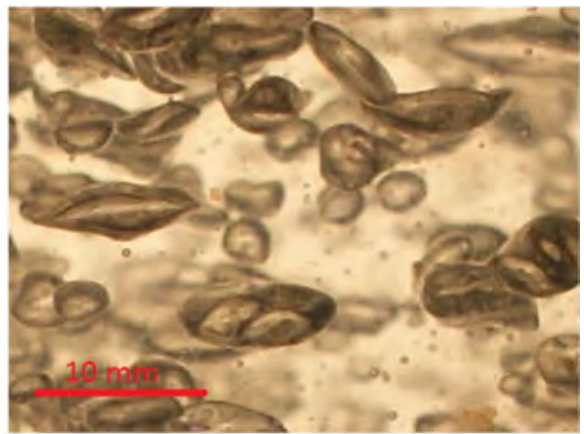

(c) $c_{M E G}=0.1 \%-\log _{10}(M o)=-10.7676$

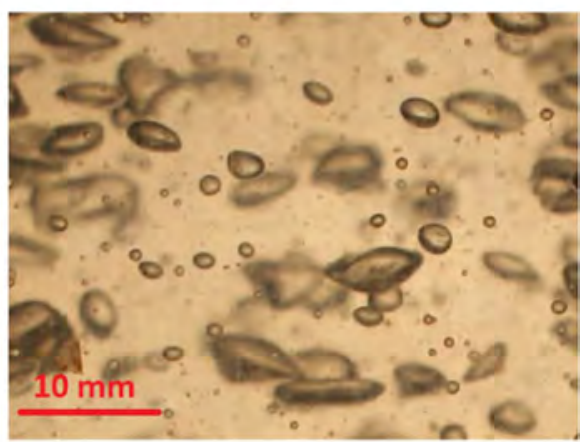

(f) $c_{M E G}=1 \%-\log _{10}(M o)=-10.721$

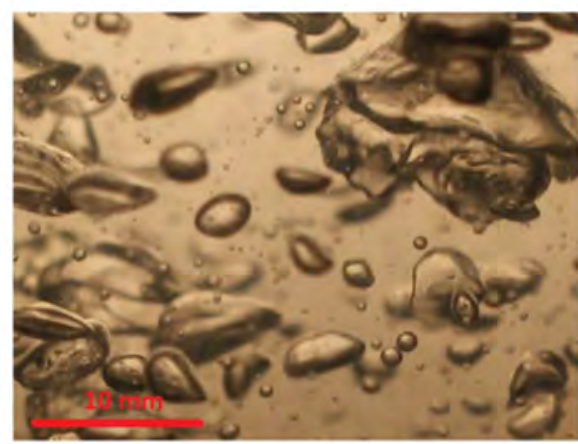

(i) $c_{M E G}=10 \%-\log _{10}(M o)=-10.2703$

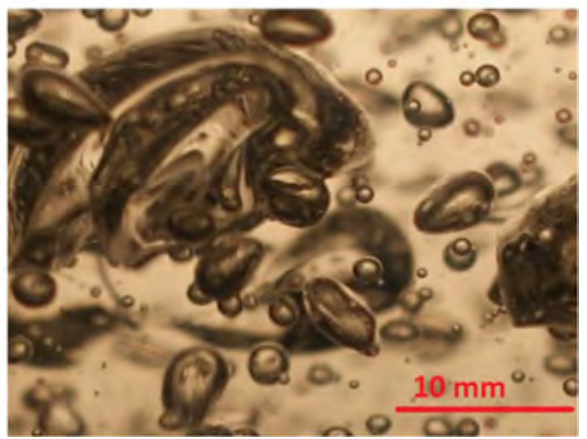

(I) $C_{M E G}=80 \%-\log _{10}(M o)=-6.54542$

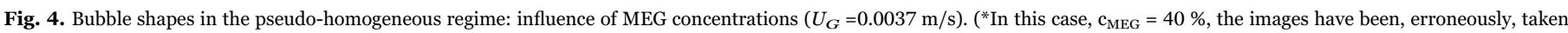
after the gas holdup measurements at high gas velocities without waiting for a sufficient time; for this reason, these data cannot be compared with the other).

holdup would increase linearly with the gas flow rate. However, the coupling between the phases causes deviations from linearity (see ref. (Ruzicka et al., 2003)). In the homogeneous flow regime, the hindrance reduces the bubble velocity, thus increasing the gas holdup, whereas in the transition flow regime, the presence of large bubbles along with the enhanced circulations (which increase bubble velocity) result in a decrease of the gas holdup and cause the gas holdup to decrease less than proportionally to the gas flow rate. The reader may also refer to the comparison between the gas holdup and the local optical probe data proposed in (Besagni and Inzoli, 2016c) for a further analysis of this behavior.

Regarding the air-water-MEG system, the gas holdup has turned out to continuously increase by increasing the MEG concentration up to $c_{M E G}=5 \%-\mu_{L}=1.01 \mathrm{mPa} \cdot \mathrm{s}$ (Fig. $2 \mathrm{a}$ ), along with the contribution 


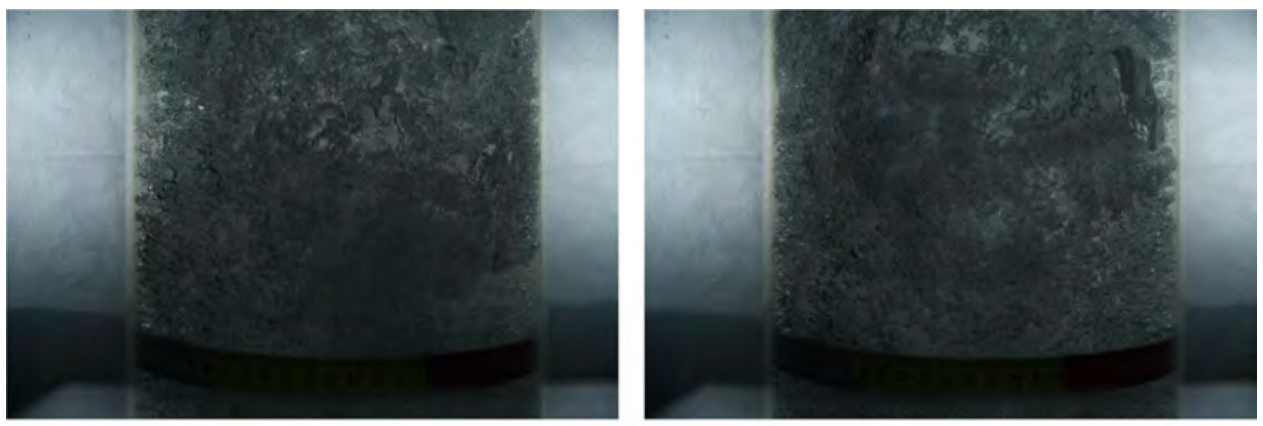

(a) $c_{\text {MEG }}=0 \%-\log _{10}(M o)=-10.7724$

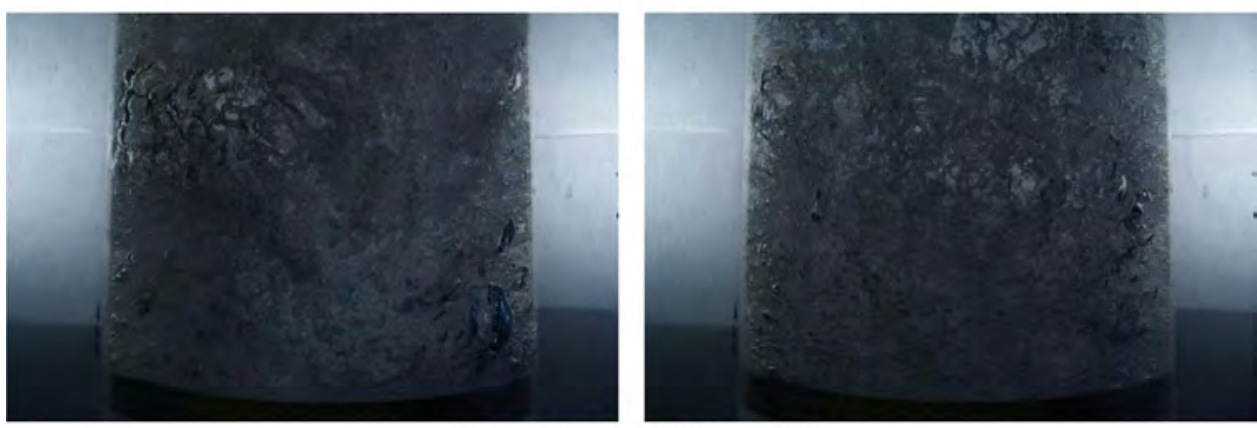

(b) $c_{\text {MEG }}=80 \%-\log _{10}(M o)=-6.54542$

Fig. 5. Flow structures in the heterogeneous regime: influence of $M E G$ concentrations $\left(U_{G}=0.1665 \mathrm{~m} / \mathrm{s}\right)$.

of small bubbles (Fig. 3; Fig. 4a, Fig. 4b, Fig. 4c and d; Section 3.3), in agreement with the visual observations of Godbole et al. (1982). This behavior is clearly observed in Fig. $4\left(U_{G}=0.0037 \mathrm{~m} / \mathrm{s}\right)$ and lies in the reduced coalescence rate between bubbles of the aqueous solutions up to $c_{M E G}=5 \%$ (as further discussed in Section 3.3): by increasing the MEG concentration up to $c_{M E G}=5 \%$ (Fig. $4 \mathrm{~d}$ ), a larger number of small bubbles is observed. An interesting aspect is the non-linearity of the viscous solution effect upon the gas holdup: the relative distance between the gas holdup curves decreases while increasing the MEG concentration. Indeed, the gas holdup curves corresponding to $c_{M E G}$ $=0.05 \%$ and $0.1 \%$ are shifted to considerably higher $\varepsilon_{G}$ values in comparison to the $c_{M E G}=0 \%$ gas holdup curve, while the relative distance between the curves decrease while increasing $c_{M E G}$ (i.e., corresponding to $c_{M E G}=0.5 \%, 0.75 \%$ and $1 \%$ ). However, as better shown in Fig. 2b, if the concentration is further increased from $c_{M E G}$ $=5 \%-\mu_{L}=1.01 \mathrm{mPa}$ s to $c_{M E G}=80 \%-\mu_{L}=7.97 \mathrm{mPa} \cdot \mathrm{s}$, the gas holdup decreases. For this last concentration, the gas holdup curve lies even below that obtained for pure water. Indeed, increasing the viscosity, the tendency to coalescence prevails, creating large cap-bubbles (Fig. 3, Fig. $4 \mathrm{i}, \mathrm{j}, \mathrm{k}$ and $\mathrm{l}$ ) rising the column at a higher velocity, thus reducing the gas holdup: this behavior is first observed at $c_{M E G}=10 \%$ (Fig. $4 \mathrm{i}$ ), and becomes even more obvious at $c_{M E G}=80 \%$ (Fig. $4 \mathrm{k}, \mathrm{j}$ ), where large cap-bubbles are observed across the vertical development of the bubble column. A similar behavior was also observed in an early study by Wilkinson et al. (Wilkinson et al., 1992) and in more recent studies (Rabha et al., 2014). In addition, at high viscosity and high $U_{G}$ "coalescence-induced" bubbles (Fig. 5b) have been observed which rise along the column, occupying a non-negligible part of the column cross-section (Figs. 3 and 5b). In particular, Fig. 5 compares the flow structures in the transition flow regime between the air-water and $c_{M E G}=80 \%$ cases. The increase in the gas holdup at low viscosities and its consequent decrease at higher viscosities is in qualitative agreement with the findings from other authors (see, for example, refs. (Bach and Pilhofer, 1978; Eissa and Schügerl, 1975; Godbole et al., 1982; Khare and Joshi, 1990; Olivieri et al., 2011; Rabha et al., 2014; Ruzicka et al., 2003)). An interesting discussion concerning the contribution of the "coalescence-induced" bubbles to the gas holdup structure in highly viscous liquid phases, was proposed by Yang et al. (2010), to whom the reader should refer.

Our experimental observations (Fig. 2a and b) confirm the existence of the "dual effect of viscosity over the gas holdup" that has been postulated in the Introduction and that has been formulated on the basis of a literature survey (mostly on small-diameter bubble columns). As expected, all cases corresponding to "low viscosities" are characterized by higher gas holdups than those of the air-water system; conversely, "moderate/high viscosities" are characterized by lower gas holdups. Further studies will be devoted to generalize these results and to define the exact boundaries for "low viscosities" and "moderate/ high viscosities". Other studies reporting similar trends for the gas holdup curve, have mainly concerned smaller bubble columns, but to the authors' opinion the proposed mechanisms may also apply to the large-diameter and large-scale bubble column studied in this work: (i) at low viscosities, the coalescence is limited (a larger number of small bubbles has been observed in this study, Fig. 4d; see Section 3.3), and the large drag force reduces the bubble rise velocity, causing an increase in the gas holdup; (ii) at higher viscosities, the tendency to coalescence prevails, creating large bubbles rising the column (as observed in Fig. 4k and l; see Section 3.3) at a higher velocity, thus reducing the gas holdup. These concepts are further discussed and motivated within Section 3.3.1. It is interesting that the present bubble column was built taking into account the well-known scale-up criteria for bubble column reactors (as explained in the Introduction): this may suggest that the present phenomena may also apply in industrial-scale bubble columns. ${ }^{1}$ When considering industrial applications, it is interesting to point-out that a change in the liquid phase may also be used to study an increase in temperature $(T)$ due to the dependency of liquid viscosity upon temperature: an increase in $T$ reduces the viscosity. A brief survey of the influence of the temperature over the gas holdup was proposed in refs. (Besagni, 2016; Leonard et al., 2015; Rollbusch et al., 2015) and, in future studies, it would be interesting to investigate whether these results may be interpreted using the dual

${ }^{1}$ This statement is based on the scale-up criteria by Wilkinson et al. (1992), that are widely accepted. Future studies should be devoted to extend and validate the scale-up criteria listed in the introduction for viscous solutions. 


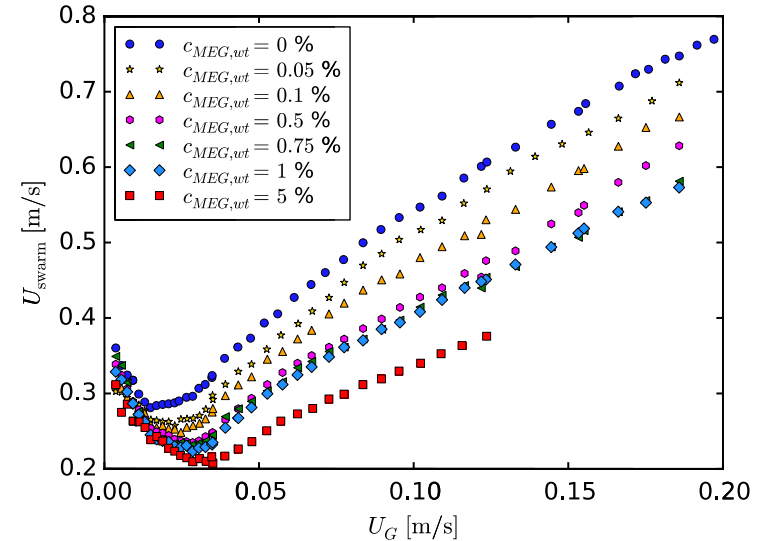

(a) Swarm velocity plot

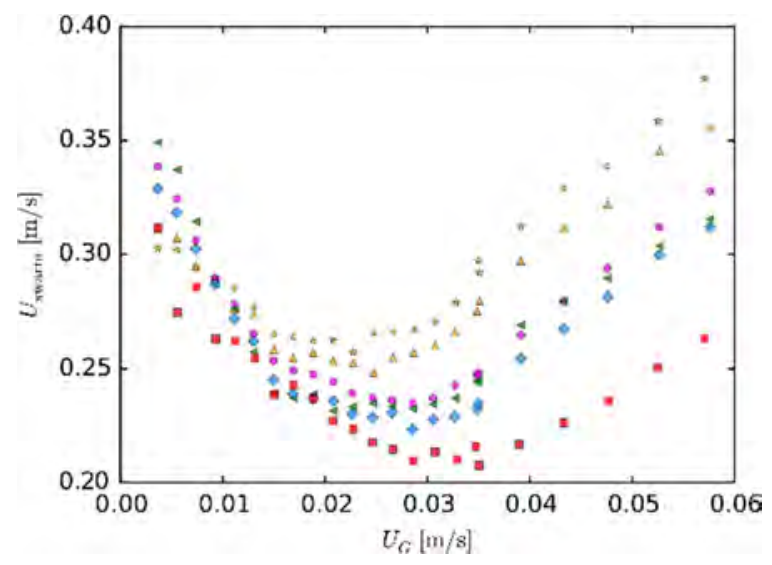

(b) Focus on the transition point

Fig. 6. Swarm velocity: influence of MEG concentration ("low viscosities").

effect of viscosity.

\subsubsection{Comparison with the dual effect observed in binary systems} with active compounds

Other researchers observed a dual effect on the gas holdup when studying binary systems with active compounds (both organic-i.e., alcoholic solutions-and inorganic substances-i.e., salts). Therefore, in this section, a brief review of these results is presented to propose a critical comparison with our experimental results and to outline future studies.

The organic substances (also called "positive surfactants") are attracted to the bubble interface where they adsorb positively and lower the surface tension. Guo et al. (2016) studied a small-diameter $\left(d_{c}=0.1 \mathrm{~m}\right)$ and small-scale $\left(H_{c}=1.8 \mathrm{~m}\right)$ bubble column by using nitrogen as the gas phase and various solutions of ethanol and $n$ butanol as the liquid phase. They found that the gas holdup first increases rapidly, and then decreases slowly when further increasing the ethanol concentration. The authors related the change in the gas holdup to the effect of the alcoholic solution on the "coalescenceinduced" and "non-coalescence-induced" bubbles. Indeed, the increasing ethanol concentration mainly changed the gas holdup of "noncoalescence-induced" bubbles but slightly affected the gas holdup of the "coalescence-induced" bubbles. Similar results were presented by Syeda et al. (2002) by using an experimental apparatus consisting of two sieve plates at a distance of $0.61 \mathrm{~m}$, which was developed to simulate a distillation tray, and with air at a fixed flow rate of $U_{G}$ $=0.32 \mathrm{~m} / \mathrm{s}$. The authors observed a peak when plotting the gas holdup as a function of the mole fraction of one of the two components in the binary systems of methanol-water, 2propanol-water, ethylene glycolwater and methanol-2propanol. A similar variation of the gas holdup

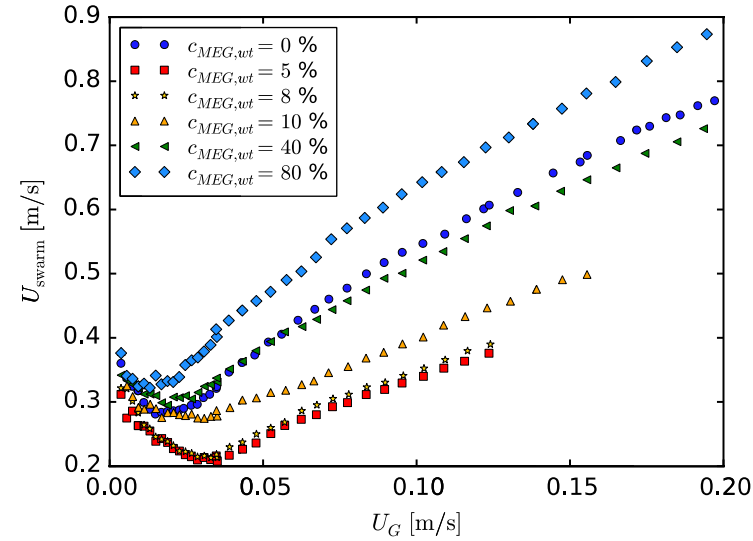

(a) Swarm velocity plot

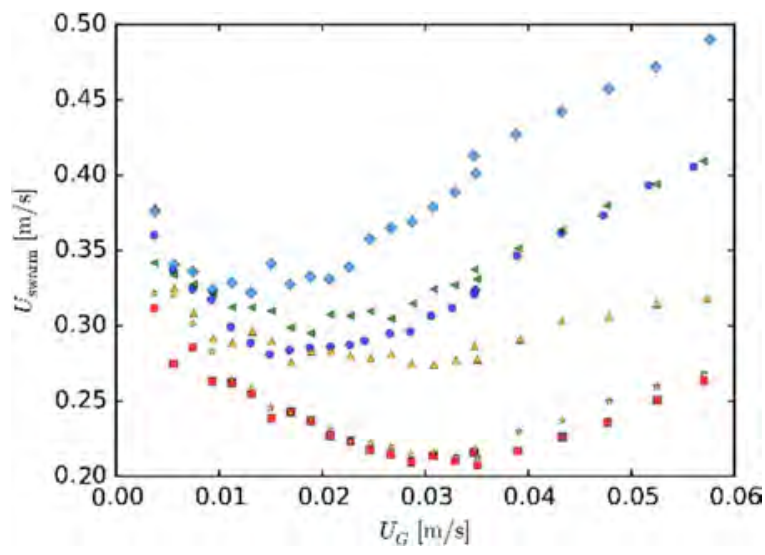

(b) Focus on the transition point

Fig. 7. Swarm velocity: influence of MEG concentration ("moderate/high viscosities").

with the composition was also observed by Bhaga et al. (Bhaga et al., 1971) in hydrogen gas holdup data for the ethanol-toluene system and a number of binary organic mixtures: with the exception of the tolueneethylbenzene and acetone-benzene pairs, in all other cases, an increased gas holdup was observed at intermediate concentrations and for the ethanol-toluene system, the maximum resulted in being sharper and shifting toward low ethanol concentrations. A similar result was presented by Shah et al. (1985), who investigated the entire range of concentrations for the ethanol-water system. All these studies (Bhaga et al., 1971; Guo et al., 2016; Shah et al., 1985; Syeda et al., 2002) explained the trend of the gas holdup with respect to the mole fraction of one of the two components in the liquid mixture on the basis of the dynamic surface tension model by Andrew (1960).

The inorganic substances (also called "negative surfactants") are repelled from the bubble interface where they adsorb negatively and cause small increase of the surface tension. Ruzicka and co-authors (Orvalho et al., 2009; Ruzicka et al., 2008) $\left(d_{c}=0.14 \mathrm{~m}, H_{\boldsymbol{O}}=0.4\right.$, perforated plate, $d_{o}=0.5 \mathrm{~mm}$ ) observed a dual effect of inorganic compounds (salts) on the gas holdup and flow regime transition in a bubble column, with respect to the electrolyte concentration. In particular, Ruzicka and co-authors (Orvalho et al., 2009; Ruzicka et al., 2008) analyzed their results in terms of the critical concentration of the inorganic compound, which is defined as the concentration of the non-coalescent media above which the bubble coalescence is drastically reduced. The reader may refer to our previous paper (Besagni and Inzoli, 2015) as well as to refs. (Orvalho et al., 2009; Ruzicka et al., 2008) for a literature survey on this topic (i.e., the influence of inorganic compounds on the gas holdup). The critical concentration is a property of the inorganic compound (i.e., it is unique for each salt, (Lessard and Zieminski, 1971)), is valid for swarm of bubbles (see refs. (Craig et al., 1993; Nguyen et al., 2012)), and is not highly dependent 


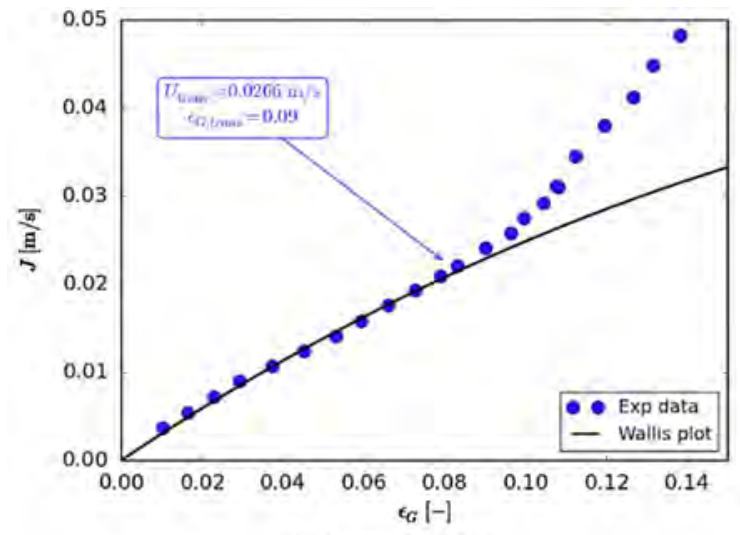

(a) $c_{M E G, w t}=0 \%$

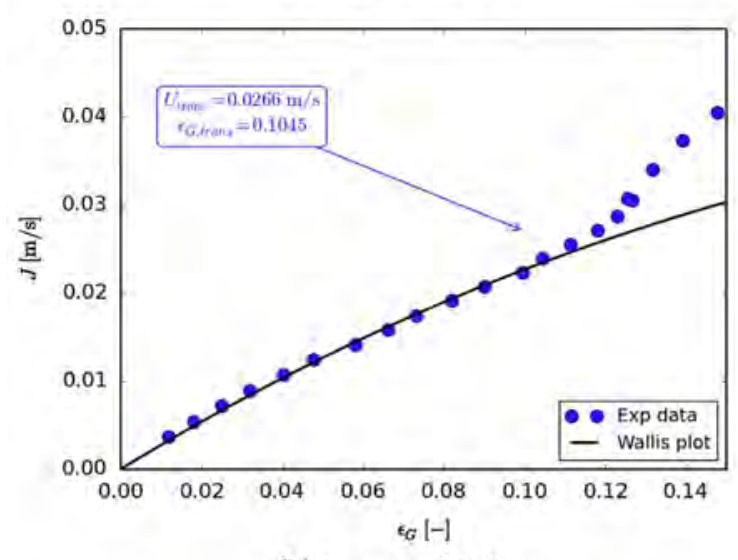

(c) $c_{M E G, w t}=0.1 \%$

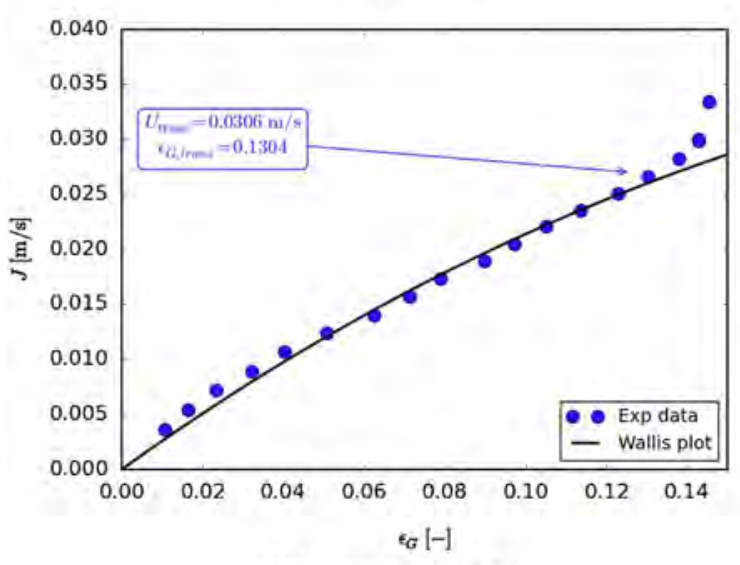

(e) $c_{\text {MEG,wt }}=0.75 \%$

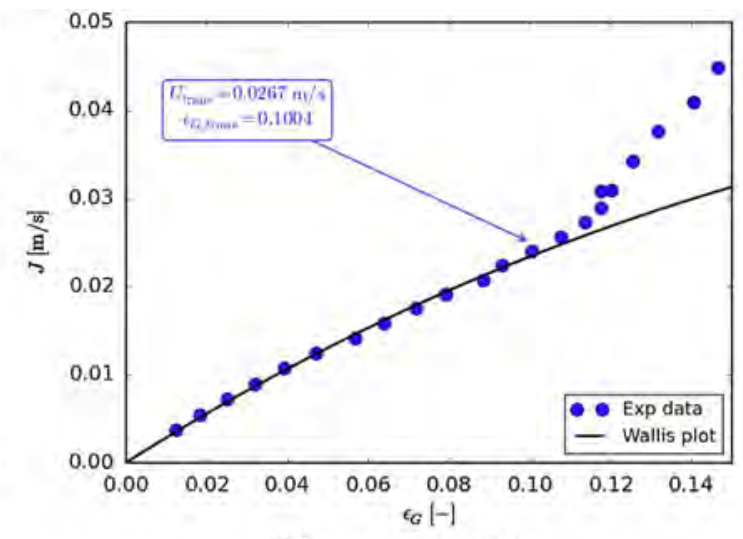

(b) $c_{M E G, w t}=0.05 \%$

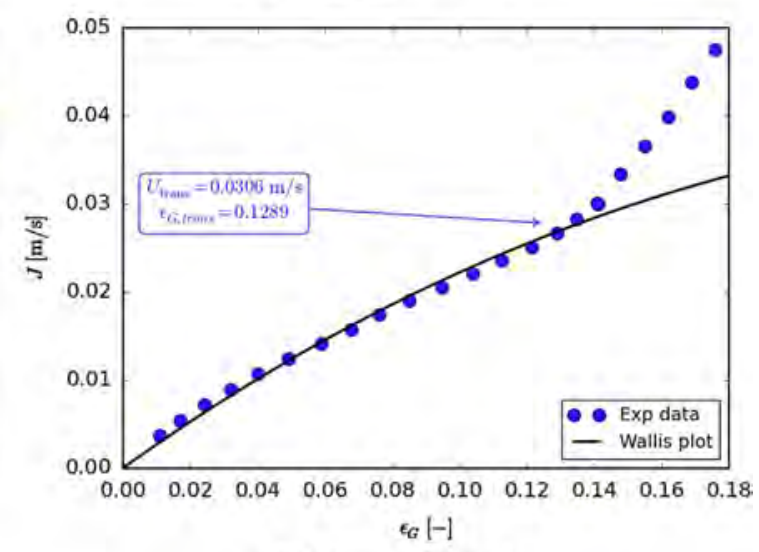

(d) $c_{M E G, w t}=0.5 \%$

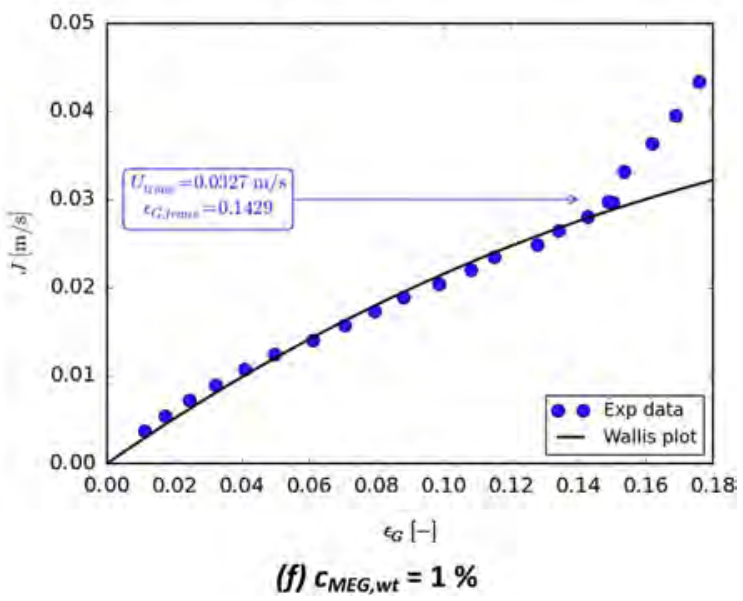

Fig. 8. Drift Flux: influence of MEG concentration ("low viscosities").

on $U_{G}$ (see refs. (Craig et al., 1993; Nguyen et al., 2012)); it is worth noting that Ribeiro Jr. and Mewes (Ribeiro Jr and Mewes, 2007), found similar gas holdup curves regardless of the nature of the salts for the given ratio between the concentration of the non-coalescent media and the critical concentration. Therefore, the critical concentration is a property of the "bubble-scale" that can be used to describe the changes in bubble column hydrodynamics (the "reactor-scale").

Further studies should be devoted to understand the common hydrodynamic properties of viscous and active compound systems, and to find a comprehensive theory/common physical model to describe all the dual effects observed: (i) the dual effect of viscosity; (ii) the dual effect of organic compounds; and (iii) the dual effect of inorganic compounds. As explained in the next section (Section 3.3), the "dual effect of viscosity on the gas holdup" lies in the changes of the BSDs.
Taking into account the physical bases of the modeling approaches used for the active compounds (i.e., "the dynamic surface tension model" and "the critical concentration for coalescence suppression"), it is reasonable to assume that all the dual effects can be described by a unique model considering the effects at the bubble interface (i.e., coalescence suppression, leading to the changes in the BSDs).

\subsection{Flow regime transition - "reactor-scale"}

The transition between the homogeneous and the transition flow regimes has been investigated by the methods presented in Section 2.2.2. The results of the swarm velocity method are presented in Figs. 6 and 7, whereas, the results of the Wallis plot method are presented in Figs. 8 and 9. Comparing the theoretical expectations with 


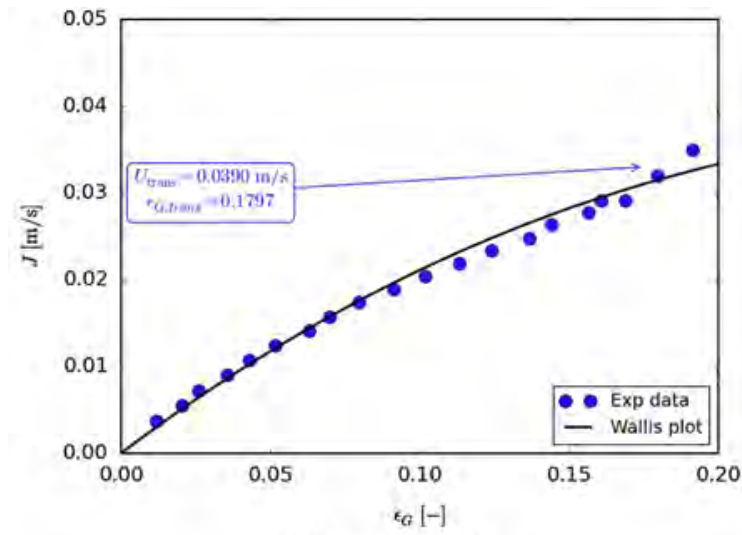

(a) $c_{M E G, w t}=5 \%$

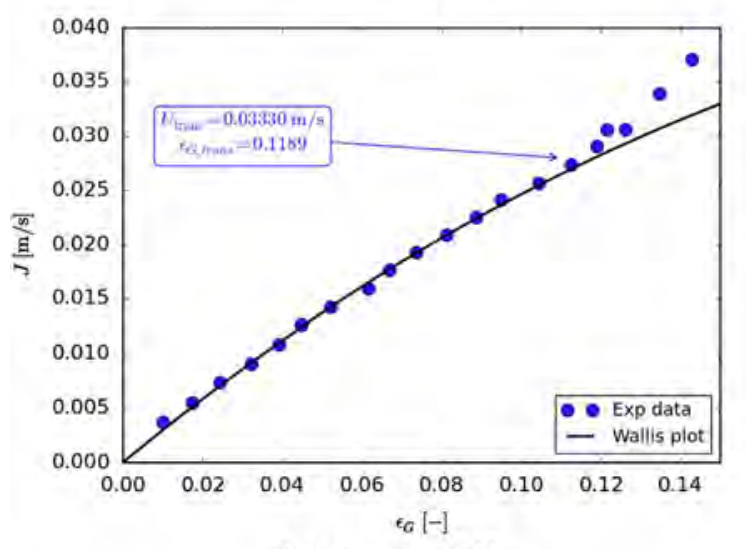

(c) $c_{M E G, w t}=10 \%$

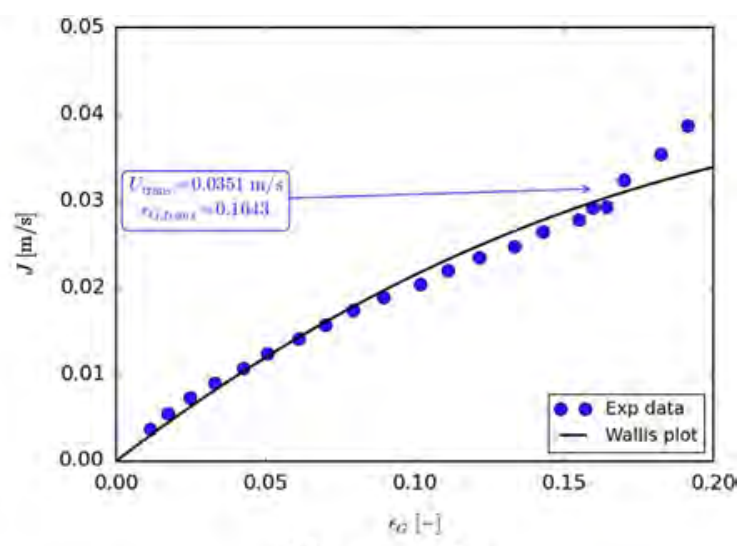

(b) $c_{M E G, w t}=8 \%$

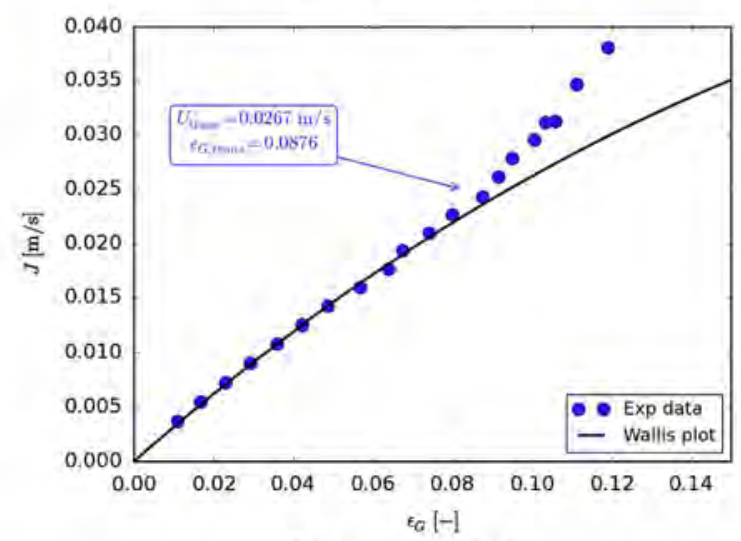

(d) $c_{M E G, w t}=40 \%$

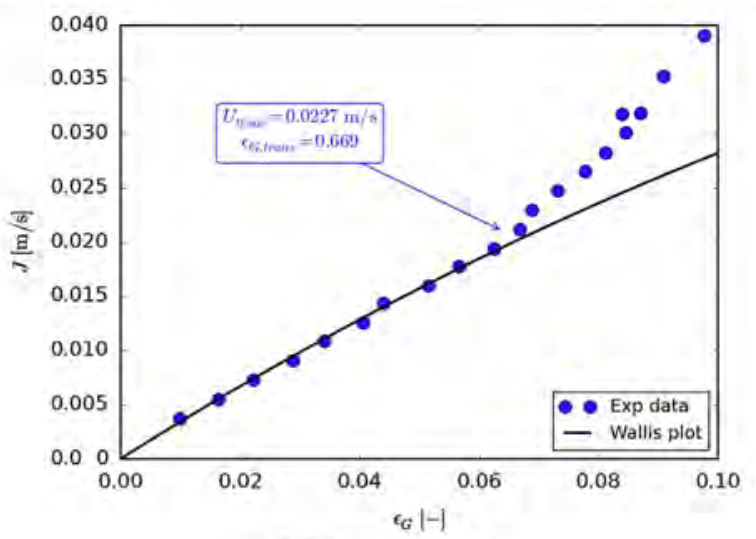

(e) $c_{M E G, W t}=80 \%$

Fig. 9. Drift Flux: influence of MEG concentration ("moderate/high viscosities").

the experimental data and following the discussion of Ruzicka (Ruzicka et al., 2003), the drift-flux plots (Figs. 8 and 9) suggest the existence of a pseudo-homogeneous/gas-maldistribution flow regime (as also confirmed by the image analysis, Section 3.3.1). The value of the transitional gas velocities and transitional gas holdup are in agreement with the two methods and, following the proposal of Ribeiro and Mewes (2007) and Besagni and Inzoli (Besagni and Inzoli, 2016b, 2016c), the transition points have been evaluated as the mean of the two values. The results of the analysis are presented in Fig. 10 and are listed in Table 2. The reader may refer to the discussion of Besagni et al. (Besagni et al., 2016b; Besagni and Inzoli, 2015, 2016b) for a detailed analysis and comparison of the flow regime transitions in air-water systems.

The experimental results indicate that the liquid phase viscosity, depending on its value, either stabilizes or destabilizes the pseudohomogeneous flow regime compared to air-water systems $\left(c_{M E G}=0 \%\right.$ : $U_{G \text {, trans }}=0.0264 \mathrm{~m} / \mathrm{s}, \varepsilon_{G}$, trans $=0.09$ ). Indeed, at "low viscosities", the pseudo-homogeneous flow regime is stabilized ( $c_{M E G}=5 \%: U_{G}$, trans $=0.039 \mathrm{~m} / \mathrm{s}, \varepsilon_{G}$, trans $=0.18$ ). In contrast, at "moderate $/$ high viscosities", the pseudo-homogeneous flow regime is destabilized $\left(c_{M E G}=80 \%: U_{G \text {, trans }}=0.023 \mathrm{~m} / \mathrm{s}, \varepsilon_{G \text {, trans }}=0.07\right)$. It is interesting that, in the present case, the pseudo-homogeneous flow regime is detected also for moderate/high viscosities, whereas other authors observed a pure-heterogeneous flow regime for $\mu_{L}>8 \mathrm{mPa} \cdot \mathrm{s}$, even with 'fine spargers' at low $U_{G}$ (Kuncová and Zahradník, 1995; Ruzicka et al., 2003; Zahradnik et al., 1997). This difference between our results and data from other authors may be related to the large-diameter bubble column, which reduces the influence of the cap-bubbles over the 


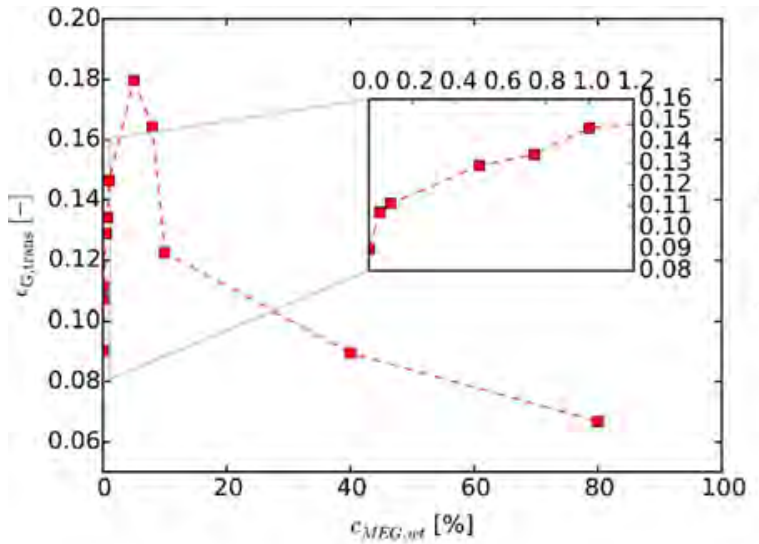

(a) $\varepsilon_{G, \text { trans }}$

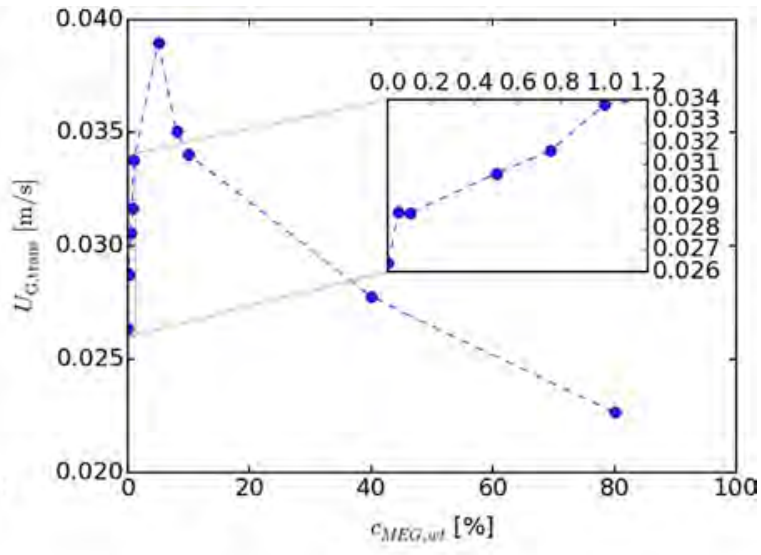

(b) $U_{G, \text { trans }}$

Fig. 10. Relation between the regime transition and the MEG concentration.

Table 2

Flow regime transition points for the system air-MEG aqueous solution.

\begin{tabular}{|c|c|c|c|c|c|c|}
\hline \multirow[t]{2}{*}{$\begin{array}{l}\mathbf{c}_{\text {MEG }} \\
{[\% w t]}\end{array}$} & \multicolumn{2}{|c|}{$\begin{array}{l}\text { Swarm velocity } \\
\text { method }\end{array}$} & \multicolumn{2}{|c|}{ Wallis Plot method } & \multicolumn{2}{|c|}{ Transition points } \\
\hline & $\begin{array}{l}U_{\text {trans }} \\
{[\mathrm{m} / \mathrm{s}]}\end{array}$ & $\begin{array}{l}\varepsilon_{G}, \\
\text { trans } \\
{[-]}\end{array}$ & $\begin{array}{l}U_{\text {trans }} \\
{[\mathrm{m} / \mathrm{s}]}\end{array}$ & $\begin{array}{l}\varepsilon_{G}, \\
\text { trans } \\
{[-]}\end{array}$ & $\begin{array}{l}U_{\text {trans }} \\
{[\mathrm{m} / \mathrm{s}]}\end{array}$ & $\begin{array}{l}\varepsilon_{G,}, \\
\text { trans } \\
{[-]}\end{array}$ \\
\hline 0 & 0.0262 & 0.0903 & 0.0266 & 0.0901 & 0.0264 & 0.0902 \\
\hline 0.05 & 0.0308 & 0.1137 & 0.0267 & 0.1005 & 0.0288 & 0.1071 \\
\hline 0.1 & 0.0308 & 0.1182 & 0.0266 & 0.1045 & 0.0287 & 0.1113 \\
\hline 0.5 & 0.0306 & 0.1289 & 0.0306 & 0.1289 & 0.0306 & 0.1289 \\
\hline 0.75 & 0.0327 & 0.1379 & 0.0306 & 0.1304 & 0.0316 & 0.1342 \\
\hline 1 & 0.0349 & 0.1501 & 0.0327 & 0.1429 & 0.0338 & 0.1465 \\
\hline 5 & 0.0390 & 0.1797 & 0.0390 & 0.1797 & 0.0390 & 0.1797 \\
\hline 8 & 0.0351 & 0.1643 & 0.0351 & 0.1643 & 0.0351 & 0.1643 \\
\hline 10 & 0.0350 & 0.1261 & 0.0330 & 0.1189 & 0.0340 & 0.1225 \\
\hline 40 & 0.0288 & 0.0915 & 0.0267 & 0.0876 & 0.0277 & 0.0895 \\
\hline 80 & 0.0227 & 0.0669 & 0.0227 & 0.0669 & 0.0227 & 0.0669 \\
\hline
\end{tabular}

whole column hydrodynamics (i.e., Fig. $4 \mathrm{k}$ and $\mathrm{l}$ : the dimensions of the cap-bubbles is lower compared to the cross section of the pipe). Our experimental observations are in agreement with the findings of Ruzicka et al. (2003), Olivieri et al. (2011) and Rabha et al. (2014). Ruzicka et al. (2003) have found that the homogeneous flow regime is stabilized for viscosities in the range of $\mu_{L}=1-3 \mathrm{mPa} \mathrm{s}$, whereas it is destabilized for viscosities in the range of $\mu_{L}=3-22 \mathrm{mPa} \mathrm{s}$, which agrees with our results. Olivieri et al. (Olivieri et al., 2011) investigated the flow regime transition between (i) the homogeneous and the vertical spiral flow regimes and (ii) the vertical-spiral and heterogeneous flow regimes. These flow regimes can be interpreted as the homogeneous, transition and heterogeneous flow regimes (as they have been defined in the Introduction). They (Olivieri et al., 2011) found a stabilization of the homogeneous flow regime up to $\mu_{L}=4.25 \mathrm{mPa}-\mathrm{s}$ and, for higher viscosities, a destabilization of the flow regime itself. However, the methods for the flow regime transition identification are slightly different from the ones applied in this analysis; therefore, despite the fact that the trends and values are qualitatively comparable, a quantitative evaluation is not possible. Finally, Rabha et al. (2014) found that, beyond $\mu_{L}=5.18-8.95 \mathrm{mPa} \mathrm{s}$, the homogeneous flow regime was no longer detected.

Our experimental results (Fig. 10) suggest the existence of the "dual effect of viscosity over flow transition", postulated in the Introduction and formulated on the basis of a literature survey (on small-diameter bubble columns). Taking into account the analysis proposed in Section 3.1.1., from the authors' perspective, the behavior of the gas holdup is strictly related to the homogeneous flow regime stabilization and/or destabilization. In particular, the stabilization of the homogeneous flow regime at "low viscosities" is related to the increased number of small bubbles because due to limited coalescence (Fig. 4a, b, c and d). In contrast, the stabilization of the homogeneous flow regime at "moderate/high viscosities" is related to the increased coalescence (Deckwer, 1992; Wilkinson et al., 1992) and the presence of large cap-bubbles (Fig. $4 \mathrm{k}$ and l). This is clearly displayed in Fig. 3 and in Fig. 4 and is further discussed and elaborated in Section 3.3, by demonstrating the "dual effect of viscosity over bubble size distributions".Finally, it is worth noting that in this study, the role of non-Newtonian liquid phases has not been investigated. However, some considerations can be made starting from the conclusions of Olivieri et al. (2011): the stabilization of the homogeneous flow regime with the relaxation time was interpreted by taking into account the negative wake phenomena occurring in the rear of a bubble in the case of viscoelastic fluids. The considerations proposed by Olivieri et al. (2011), along with the relationships between the flow regime transition and the gas holdup curve verified in this study, explain the higher value for the nonNewtonian solutions obtained by Godbole et al. (1982).

For a comparison with the results obtained in binary systems when using active compounds (organic and inorganic), the reader should refer to Section 3.1.2: the same considerations from before also apply here.

\subsection{Image analysis - "bubble-scale"}

In this section, the image analysis is presented to provide insight in the pseudo-homogeneous flow regime, considering five gas velocities in particular (between $U_{G}=0.0037$ and $U_{G}=0.0188 \mathrm{~m} / \mathrm{s}$ ) for different MEG concentrations. Please note that for some MEG concentrations, the image analysis was not possible. For example, we had some issues in the $c_{M E G}=40 \%$ case (in this case, the images were erroneously taken after the gas holdup measurements without waiting for a sufficient amount of time so that these data cannot be compared to the other data). Further results, for the air-water system, were presented by the authors in a previous work (Besagni and Inzoli, 2016b).

The results are presented in Figs. 4-20. Fig. 4 qualitatively displays (as previously discussed), the influence of the MEG concentration on the bubble shape in the pseudo-homogeneous flow regime. These images have been analyzed through the methods presented in Section 2.3.2 and Fig. 11 displays, quantitatively, the influence of MEG concentration and $U_{G}$ on the BSDs. Moreover, Figs. 12-20 further display the relationships between the bubble sizes and shapes for the bubbles sampled using the ellipse fitting method. In the following, the detailed analysis of these data is proposed. 


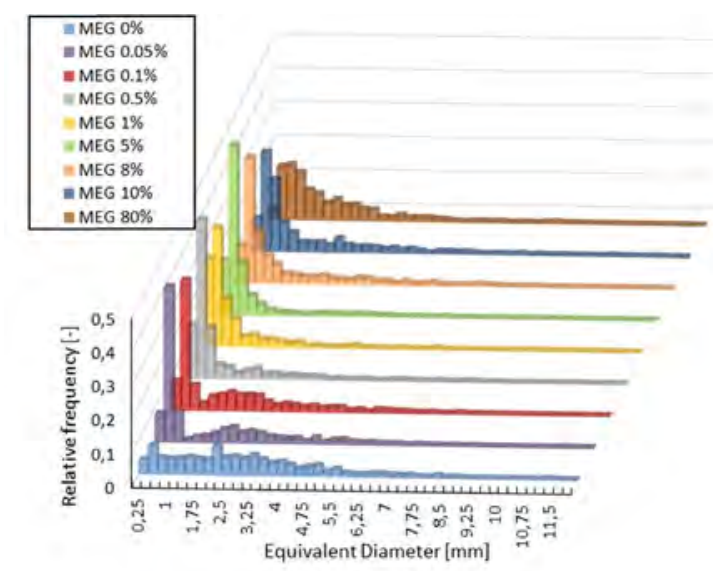

(a) $U_{G}=0.0037 \mathrm{~m} / \mathrm{s}$

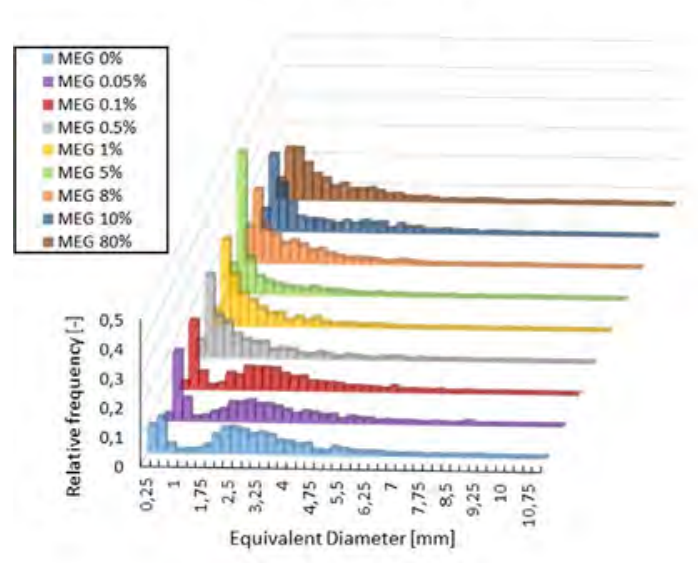

(c) $U_{G}=0.0111 \mathrm{~m} / \mathrm{s}$

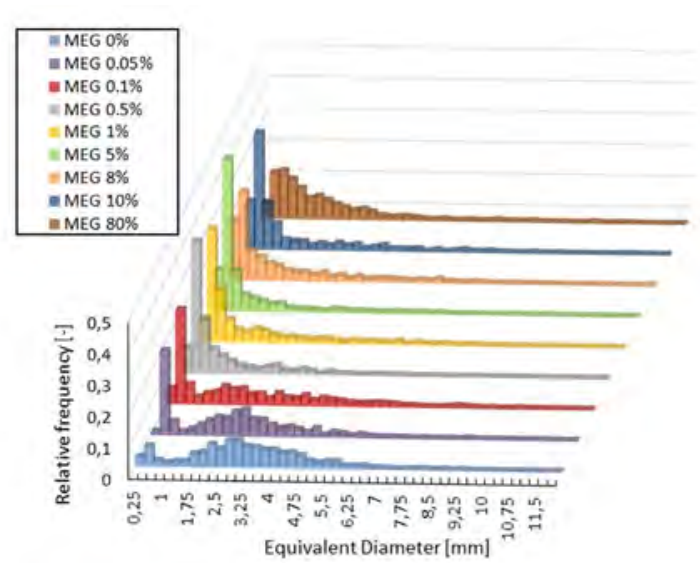

(b) $U_{G}=0.0074 \mathrm{~m} / \mathrm{s}$

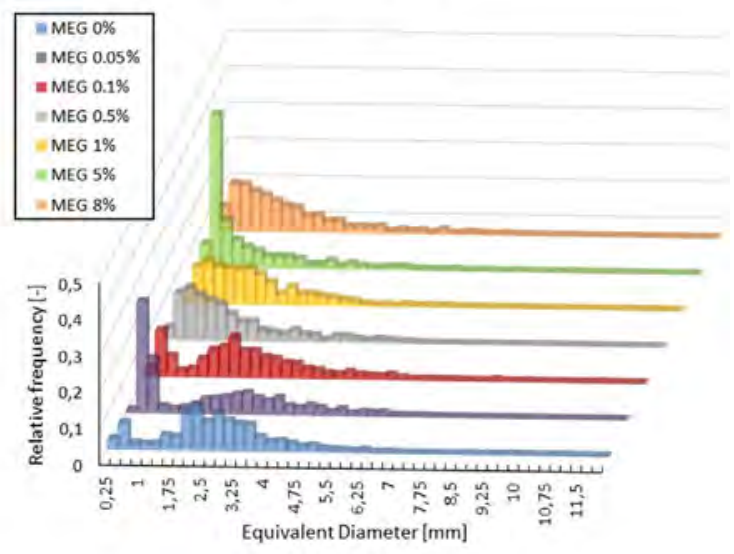

(d) $U_{G}=0.0149 \mathrm{~m} / \mathrm{s}$.

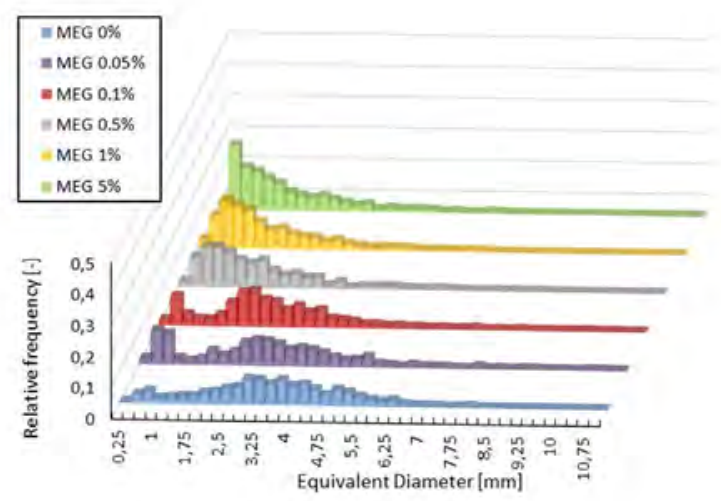

(e) $U_{G}=0.0188 \mathrm{~m} / \mathrm{s}$.

Fig. 11. Influence of MEG concentration over BSDs.

\subsubsection{Bubble size distributions}

Regardless of the MEG concentration, the BSDs cover a very wide spectrum of bubble sizes for all gas inputs (this has been also qualitatively observed in Fig. 4): the system is heavily poly-dispersed. Due to the poly-dispersed BSDs and in accordance with the discussion proposed in the Introduction, the homogeneous flow regime has been classified, within this paper, as a pseudo-homogeneous flow regime. An increase in $U_{G}$ always results in shifting the BSDs toward higher diameters, which is probably caused by the higher coalescence at higher $U_{G}$. Indeed, by increasing $U_{G}$, the bubble velocity and the gas holdup increase (see, for example, refs. (Besagni and Inzoli, 2016c) and (Besagni and Inzoli, 2016b)), thus the bubbles are closer and have more kinetic energy. Considering the air-water system, up to $U_{G}$ $=0.0149 \mathrm{~m} / \mathrm{s}$, the BSDs are bimodal: the first peak of the frequency occurs between $d_{e q}=0.5 \mathrm{~mm}$ and $1 \mathrm{~mm}$, whereas the second peak, depending on $U_{G}$, ranges between $d_{e q}=2.5 \mathrm{~mm}$ and $3.5 \mathrm{~mm}$. At $U_{G}$ $=0.0188 \mathrm{~m} / \mathrm{s}$, the BSD changes from bimodal to unimodal and shifts toward higher bubble diameters with a peak between $d_{e q}=3.5 \mathrm{~mm}$ and $4 \mathrm{~mm}$, due to the close flow regime transition (Lucas et al., 2003). Further details on the air-water system and a detailed comparison with the previous studies are given in (Besagni and Inzoli, 2016b). When increasing the MEG concentration, the BSDs become narrower and the peak of small bubbles at $d_{e q}=0.5 \mathrm{~mm}$ increases up to its maximum value at $c_{M E G}=5 \%$. This is clearly observable from the flow visualizations of Figs. 3 and 4d. For higher MEG concentrations, the BSDs become wider and the peak at $d_{e q}=0.5 \mathrm{~mm}$ has a lower frequency. At moderate/high viscosities, cap-bubbles appear in addition to the spherical-ellipsoidal bubbles: this behavior is first observed at $c_{M E G}$ 


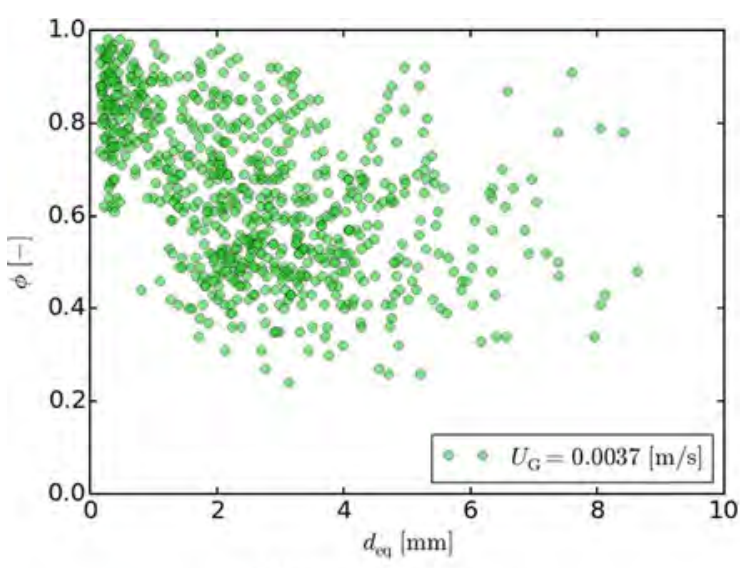

(a) $U_{G}=0.0037 \mathrm{~m} / \mathrm{s}$

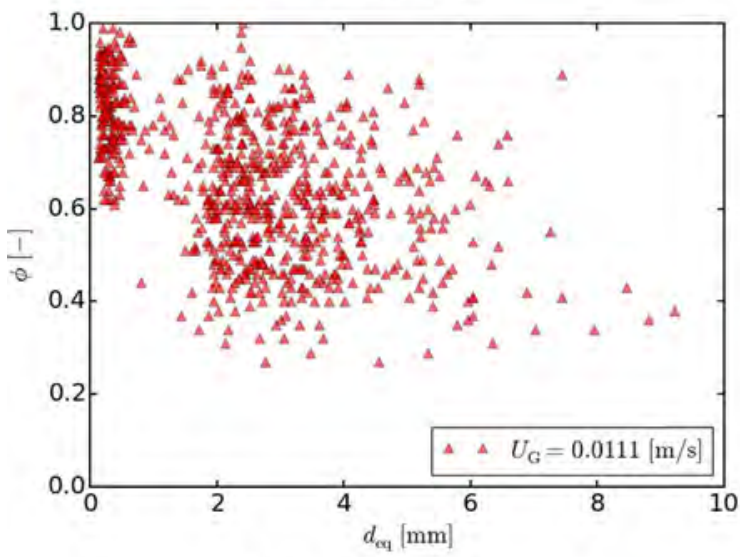

(c) $U_{G}=0.0111 \mathrm{~m} / \mathrm{s}$

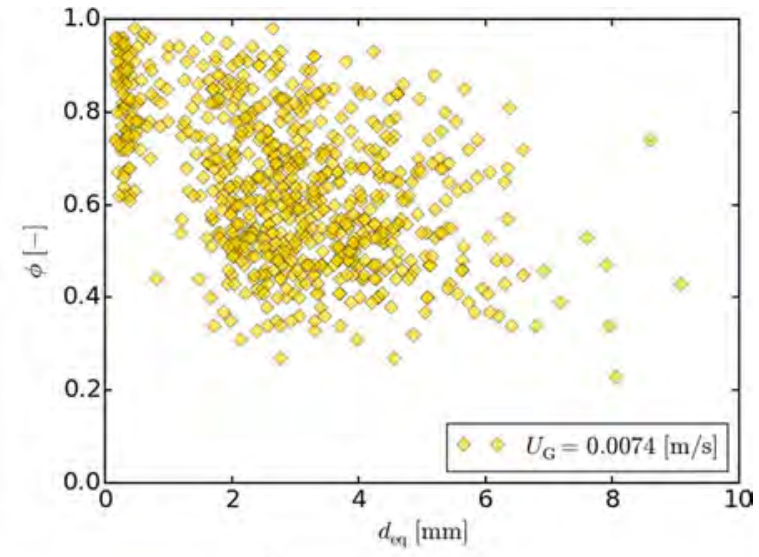

(b) $U_{G}=0.0074 \mathrm{~m} / \mathrm{s}$

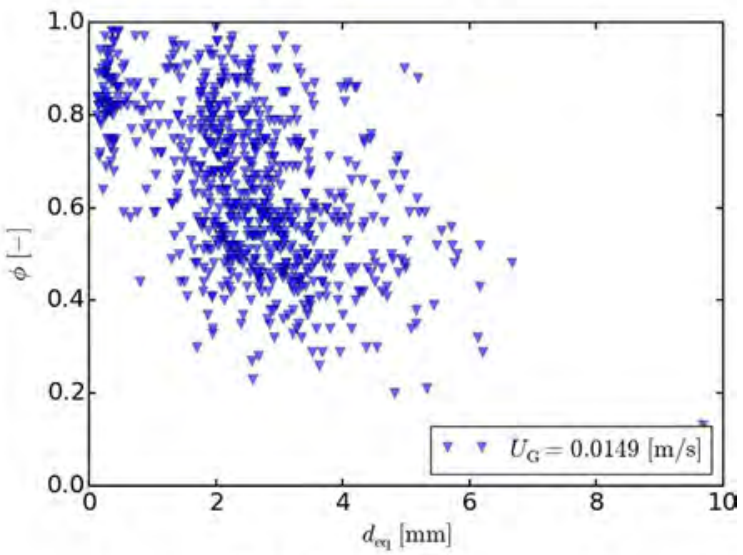

(d) $U_{G}=0.0149 \mathrm{~m} / \mathrm{s}$

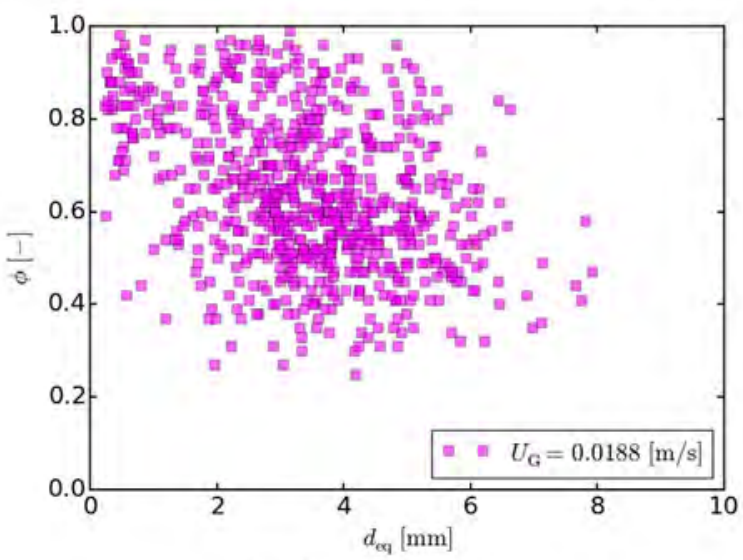

(e) $U_{G}=0.0188 \mathrm{~m} / \mathrm{s}$

Fig. 12. Relationship between the aspect ratio and the equivalent diameter $-c_{M E G}=0 \%-\log _{10}(M o)=-10.7724$ : influence of $U_{G}$

$=10 \%$ (Fig. 4i), and becomes even more obvious at $c_{M E G}=80 \%$ (Fig. 4k, j), where large cap-bubbles are observed all over the vertical development of the bubble column. Despite the low relative frequency of the cap-bubbles, their volume (and, thus, the influence on the volume fraction) is not negligible.

These results are further quantified by the data presented in Figs. $12-20$ : up to $c_{M E G}=5 \%$, the number of small and spherical bubbles increases, whereas at a higher concentration, the bubbles tend to be more distorted (please note that these data consider only ellipsoidal bubbles and neglect cap-shaped bubbles). Further comments on the data presented in Figs. 12-20 are given in Section 3.3.2 Our results are in agreement with the theoretical expectations: it is known that viscous media are characterized by a high coalescence rate (Deckwer, 1992; Kuncová and Zahradník, 1995; Wilkinson et al., 1992; Zahradnik et al., 1997) and low breakup rate (Shah et al., 1982; Wilkinson et al., 1992). For these reasons, in viscous media, a bimodal population of small and very large bubbles is typically observed (Philip et al., 1990; Rabha et al., 2014), which is in qualitative and quantitative agreement with our observations. Similar behavior was observed by Yang et al. (Yang et al., 2010) in a smaller bubble column and using a "fine sparger": they interpreted the increase in the number of small bubbles at moderate viscosities using the stabilization effect of the bubbly layers (Ruzicka et al., 2003). Indeed, moderate viscosities stabilize and increase the boundary layer thickness between the 


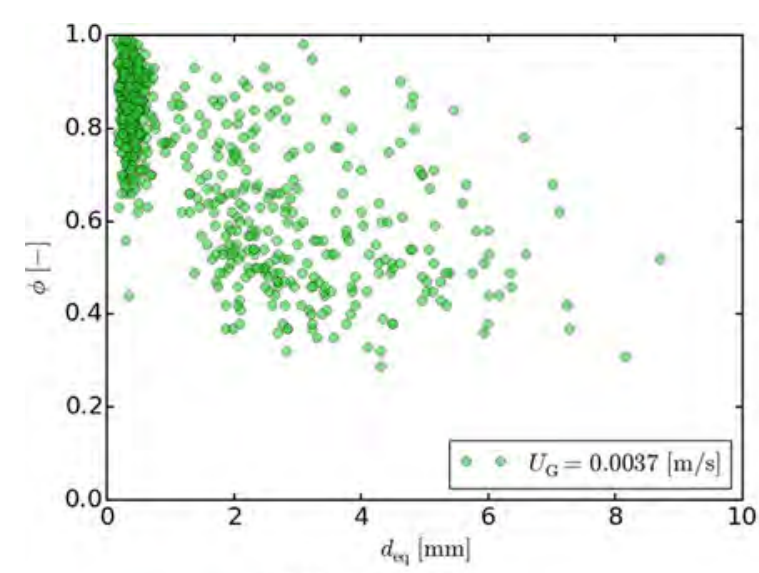

(a) $U_{G}=0.0037 \mathrm{~m} / \mathrm{s}$

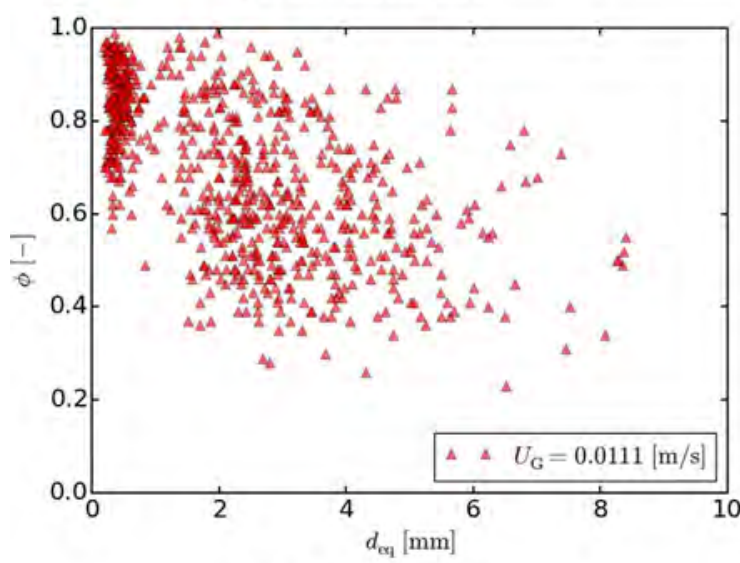

(c) $U_{G}=0.0111 \mathrm{~m} / \mathrm{s}$

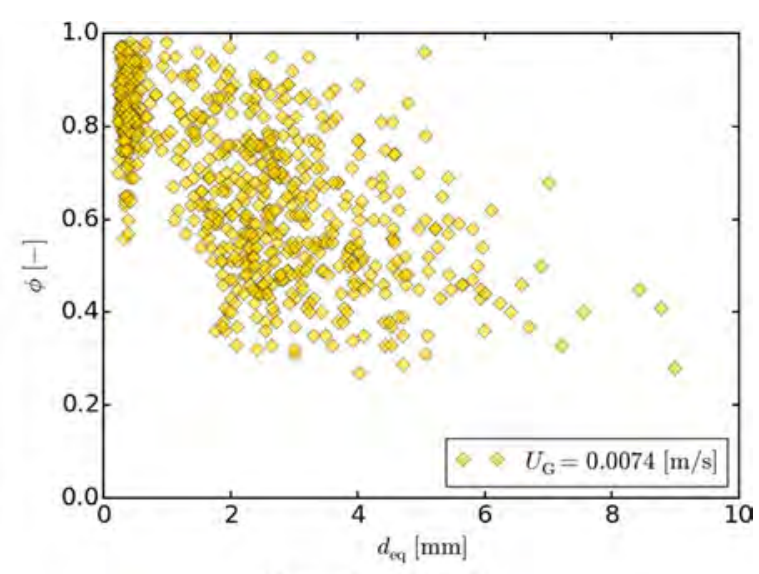

(b) $U_{G}=0.0074 \mathrm{~m} / \mathrm{s}$

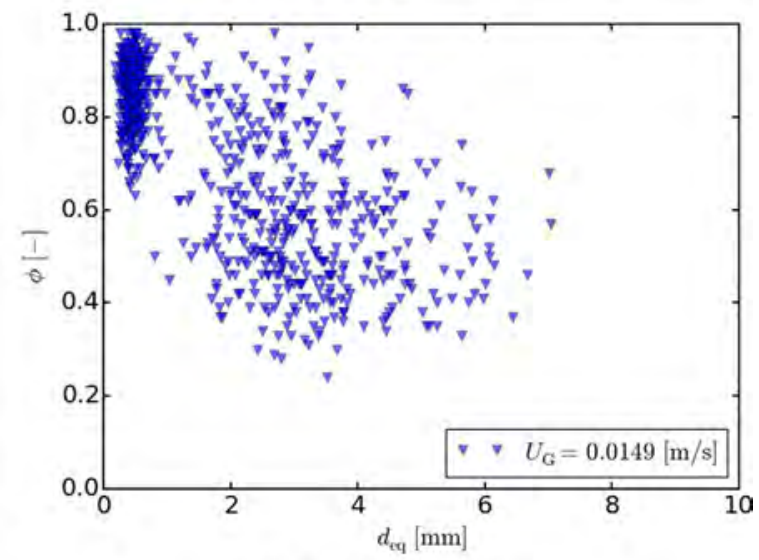

(d) $U_{G}=0.0149 \mathrm{~m} / \mathrm{s}$

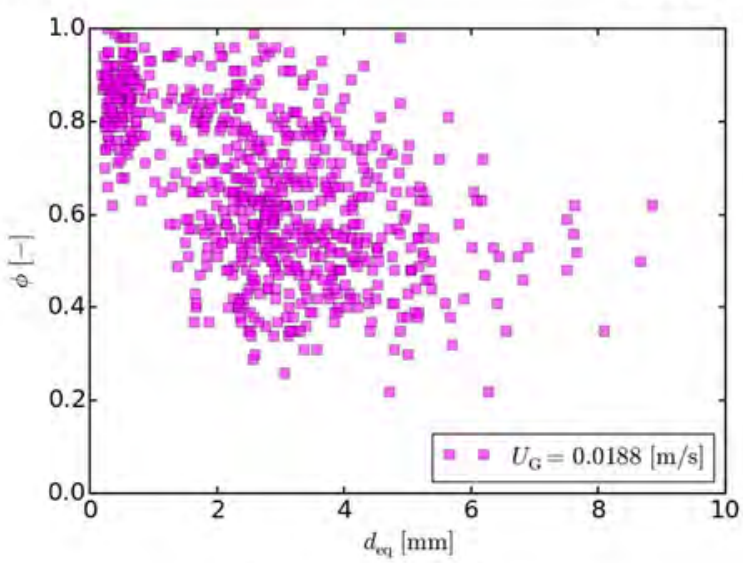

(e) $U_{G}=0.0188 \mathrm{~m} / \mathrm{s}$

Fig. 13. Relationship between the aspect ratio and the equivalent diameter $-c_{M E G}=0.05 \%-\log _{10}(M o)=-10.7697$ : influence of $U_{G}$.

bubbles, resulting in a decrease of the coalescence rate of the small bubbles (the momentum of the bubbles could not overcome the layer thickness). In contrast, at higher viscosities, the coalescence is promoted and large bubbles appear. These motivations may also apply to our system, thus explaining the physical mechanisms behind the "dual effect of viscosity" observed within this work.

It is worth noting that in the image analysis, only bubbles near the wall have been sampled. The cap-bubbles near the wall have a typical equivalent diameter in the range of 10-17 $\mathrm{mm}$. These cap bubbles are, of course, lower in number and smaller than the large ones appearing at the center of the column (see, for example, Figs. 3 and 4). It is also worth noting that, except for the very low $U_{G}$, the center of the column can not be analyzed by using the present image analysis methods. This point was also discussed in our previous study (Besagni and Inzoli, 2016b). The very large bubbles tend to migrate toward the center of the pipe, because of the lift force (Section 3.4).

Our experimental results suggest that the "dual effect of viscosity" also applies to the bubble size distributions. The quantitative study of the "dual effect of viscosity over BSDs" is further discussed and qualitatively detailed in Section 3.3.2 and Section 3.4. To the authors' best knowledge, this is the first study to observe and evaluate the dual effect of viscosity over the bubble sizes. The only previous work to observe a similar behavior was proposed by Yang et al. (Yang et al., 2010). This result is very interesting because it suggests that the "dual 


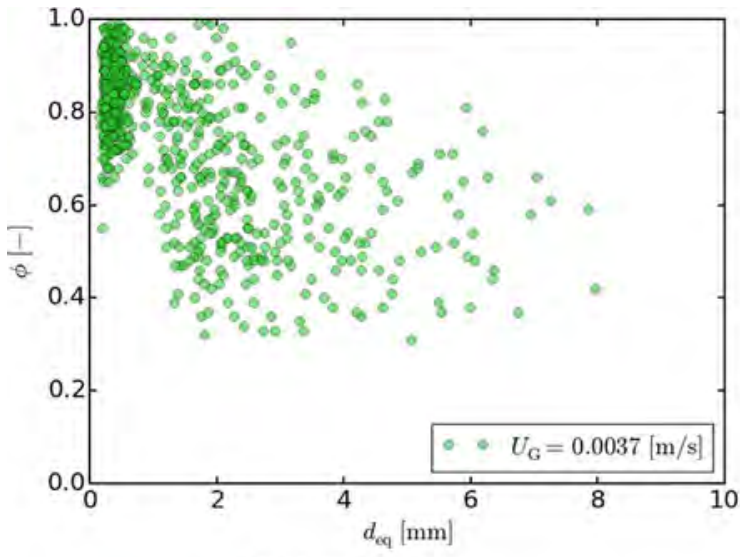

(a) $U_{G}=0.0037 \mathrm{~m} / \mathrm{s}$

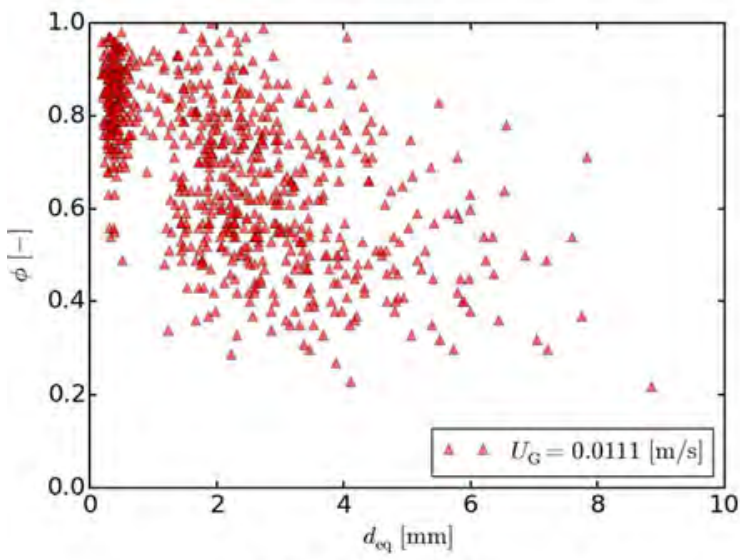

(c) $U_{G}=0.0111 \mathrm{~m} / \mathrm{s}$

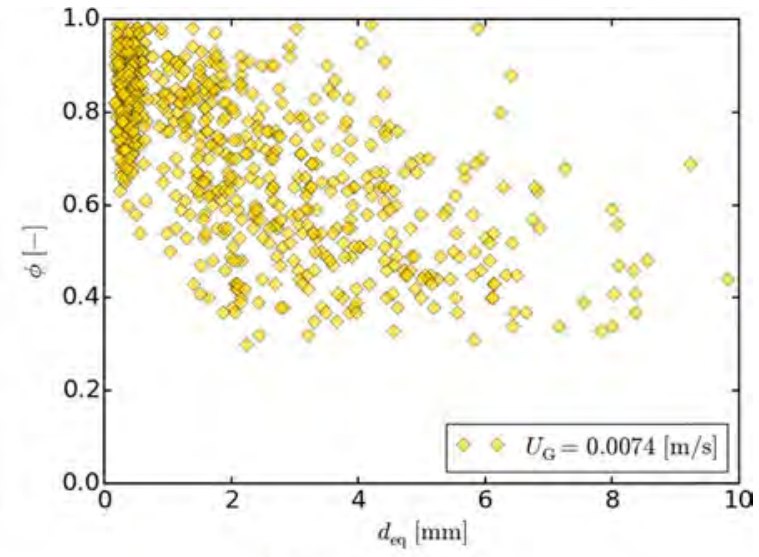

(b) $U_{G}=0.0074 \mathrm{~m} / \mathrm{s}$

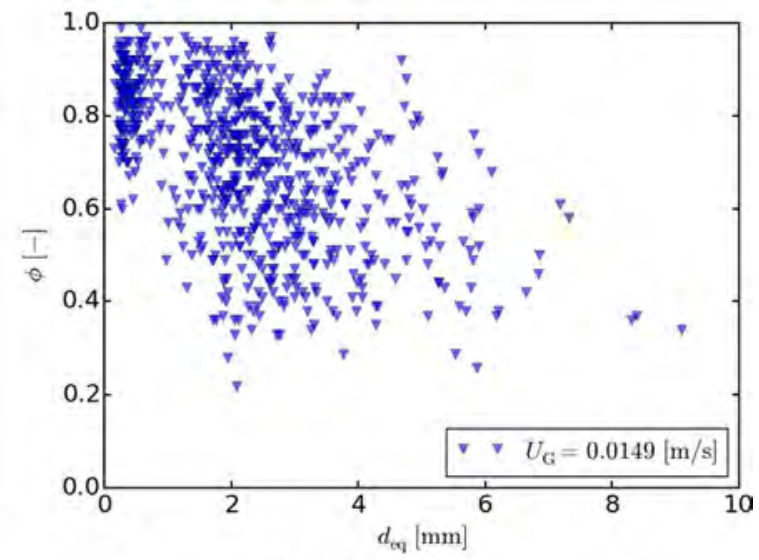

(d) $U_{G}=0.0149 \mathrm{~m} / \mathrm{s}$

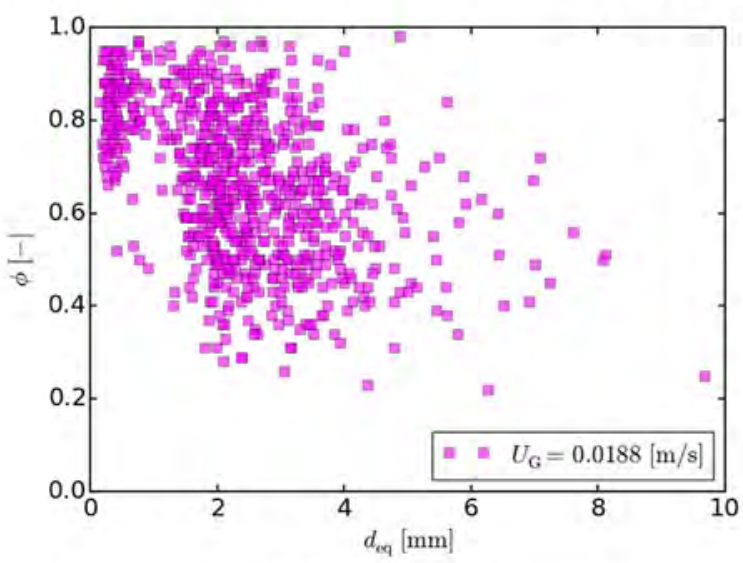

(e) $U_{G}=0.0188 \mathrm{~m} / \mathrm{s}$

Fig. 14. Relationship between the aspect ratio and the equivalent diameter $-c_{M E G}=0.1 \%-\log _{10}(M o)=-10.7676$ : influence of $U_{G}$.

effect of viscosity over BSDs" is the cause of the flow regime stabilization/destabilization and, thus, the gas holdup increases/decreases. This concept is further elaborated in Section 3.4. Generally, these results suggest that the stabilization of the homogeneous flow regime and, thus, the changes in the gas holdup are caused by the modifications to the BSDs and are induced by the coalescence suppression/enhancement mechanisms (connected to the bubble interface properties, (Firouzi et al., 2015; Liao and Lucas, 2009, 2010)), as previously observed by the authors for an aqueous solution of ethanol (Besagni et al., 2016c). The detailed relationship between the bubble interface properties and the BSDs are a matter for ongoing and future studies.

\subsubsection{Bubble shapes and aspect ratio correlation}

3.3.2.1. Bubble shapes. The relations between the bubble shapes and sizes are qualitatively displayed in Fig. 4 and are quantitatively presented in Figs. 13-21. In particular, Figs. 13-21 display the relationship between the bubble aspect ratio and the equivalent diameter for the air-water-MEG system (the $d_{e q}-\varphi$ space, as described by Besagni et al. (Besagni and Inzoli, 2016a, 2016b; Besagni et al., 2016c)). Please note that the data presented in Figs. 13-21 correspond to the bubbles sampled by using the ellipse fitting method and, therefore, the cap bubbles are neglected. It is worth noting that-owing to the point selection for ellipse fitting-the smaller 


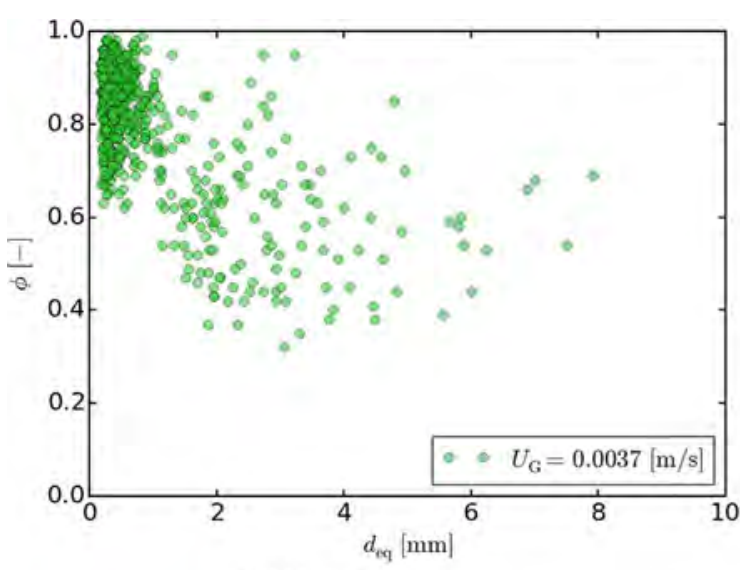

(a) $U_{G}=0.0037 \mathrm{~m} / \mathrm{s}$

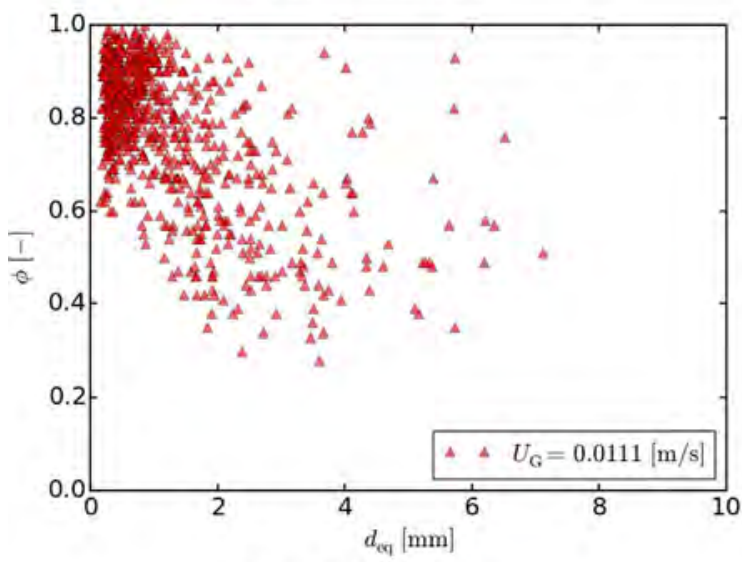

(c) $U_{G}=0.0111 \mathrm{~m} / \mathrm{s}$

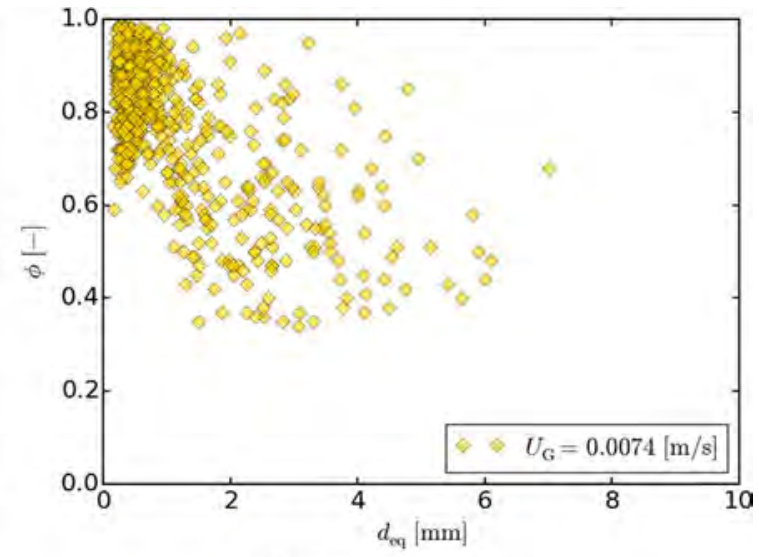

(b) $U_{G}=0.0074 \mathrm{~m} / \mathrm{s}$

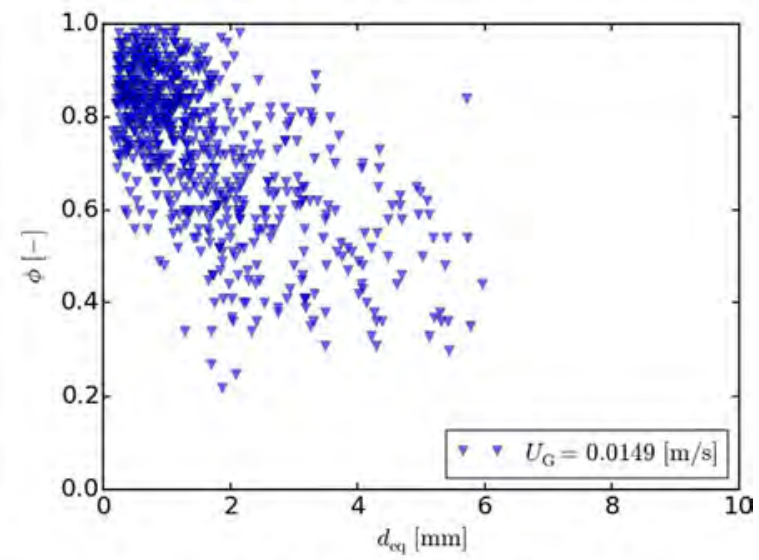

(d) $U_{G}=0.0149 \mathrm{~m} / \mathrm{s}$

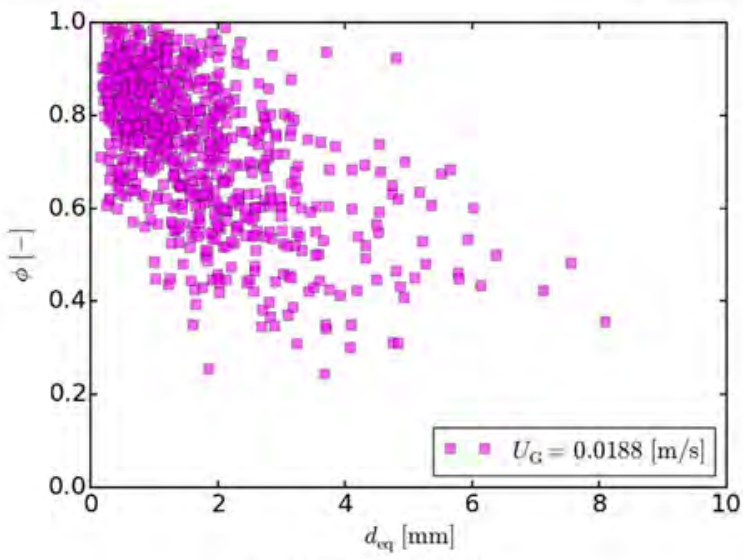

(e) $U_{G}=0.0188 \mathrm{~m} / \mathrm{s}$

Fig. 15. Relationship between the aspect ratio and the equivalent diameter $-c_{M E G}=0.5 \%-\log _{10}(M o)=-10.7466$ : influence of $U_{G}$.

the bubble, the larger the error of estimation will be (see ref. (Besagni and Inzoli, 2016a)).

The bubble aspect ratio distributions for the air-water case were presented in our previous paper (Besagni and Inzoli, 2016b), to which the reader should refer, and are briefly summarized here. In the airwater case the aspect ratio distributions up to $U_{G}=0.0147 \mathrm{~m} / \mathrm{s}$ are quite similar: approximately $6-7 \%$ of bubbles have $\varphi$ between 0.9 and $1,87-90 \%$ of the bubbles have $0.4 \leq \varphi \leq 0.9$, and $4-6 \%$ have $0.3 \leq \varphi \leq 0.4$. At $U_{G}=0.0188 \mathrm{~m} / \mathrm{s}$ there is a change in the distribution, and more significantly distorted bubbles appear. When increasing the MEG concentration up to $c_{M E G}=5 \%$ the data on the $d_{e q}-\varphi$ space moves toward the region of high aspect ratio and low dimeter bubbles and, for higher concentrations, the data are more distributed in the whole $d_{e q}-$ $\varphi$ space, as expected. These results are further quantified in the next section. These results demonstrate that a change in the liquid phase properties mainly change the relationships between bubble shapes and sizes and, thus, the BSDs. This is interesting because the $d_{e q}-\varphi$ space is determined by the properties at the bubble interface (the reader may refer, for example, to ref. (Firouzi et al., 2015): despite the fact that the topic is different, the framework is similar, as previously discussed). This result, taking into account that the "dual effect of viscosity over BSDs", supports the hypothesis that the stabilization of the homogeneous flow regime is caused by the large number of small "non- 


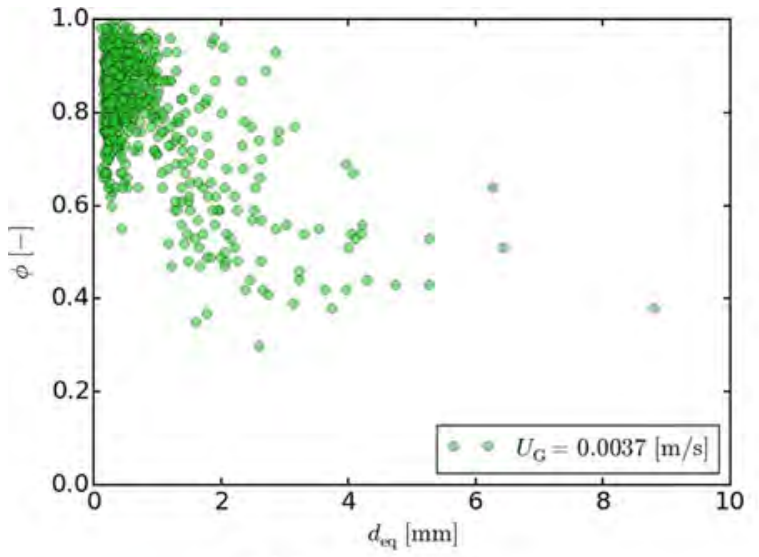

(a) $U_{G}=0.0037 \mathrm{~m} / \mathrm{s}$

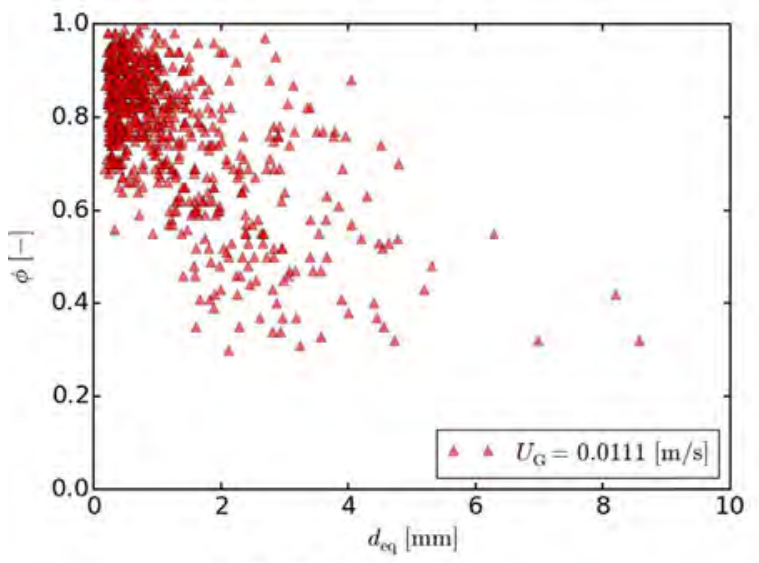

(c) $U_{G}=0.0111 \mathrm{~m} / \mathrm{s}$

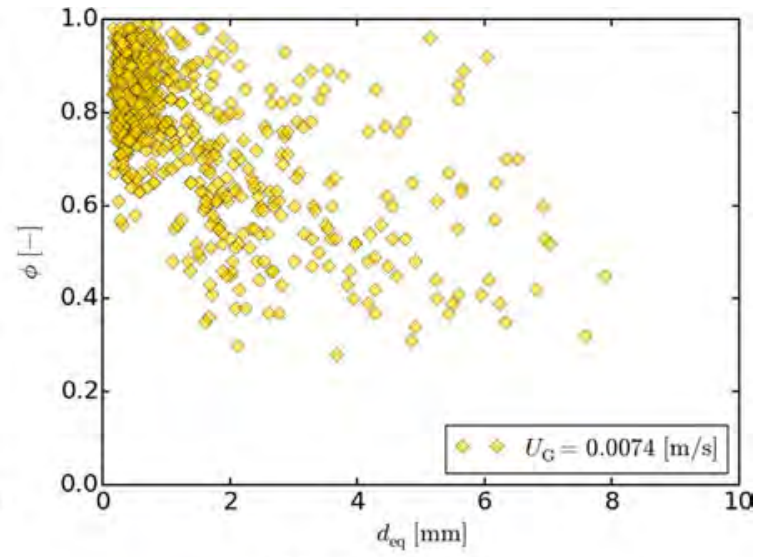

(b) $U_{G}=0.0074 \mathrm{~m} / \mathrm{s}$

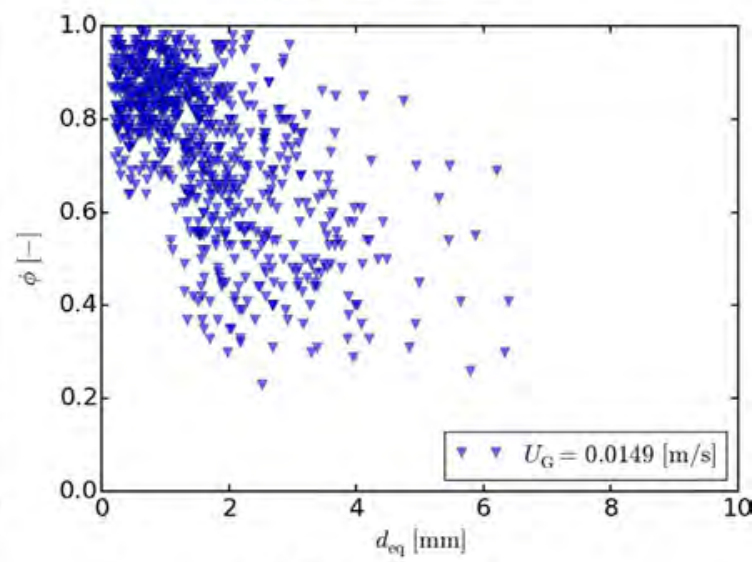

(d) $U_{G}=0.0149 \mathrm{~m} / \mathrm{s}$

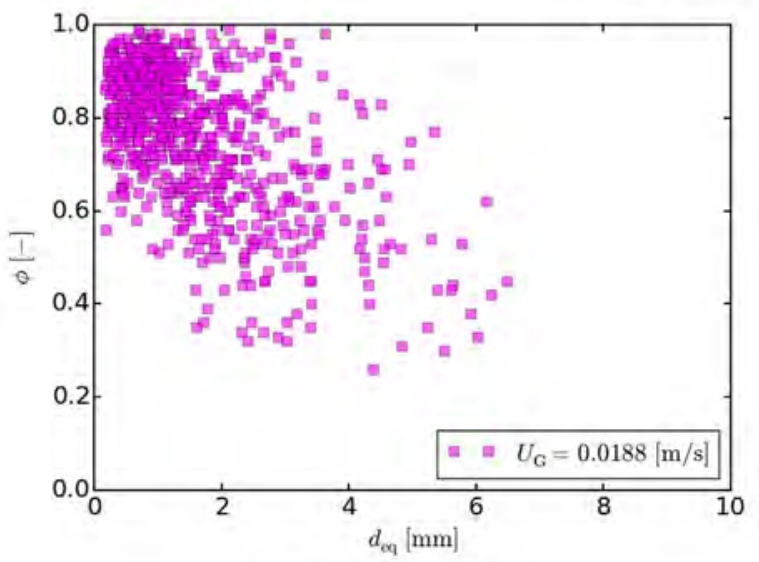

(e) $U_{G}=0.0188 \mathrm{~m} / \mathrm{s}$

Fig. 16. Relationship between the aspect ratio and the equivalent diameter $-c_{M E G}=1 \%-\log _{10}(M o)=-10.721$ : influence of $U_{G}$.

coalescence-induced bubbles". Similar results were presented in our paper concerning the effect of ethanol on bubble shapes and sizes (Besagni et al., 2016c), to whom the reader may refer for a further comparison and discussion. These data are used, in next Section, to investigate the relationship between the bubble shapes and sizes, through non-dimensional parameters (as in ref. (Aoyama et al., 2016)).

3.3.2.2. Aspect ratio correlation. The many relations between the bubble column fluid-dynamic parameters and the variables characterizing the system make it difficult to find general correlations for the precise estimation of the bubble sizes and shapes, which is the analytical relation between $d_{e q}$ (or related non-dimensional groups) and $\varphi$. Numerous correlations are available in literature, although due to the fluid dynamic complexity of the problem none of them can be either considered of general validity or applied to a wide range of geometrical parameters and operating conditions. Most of the studies have dealt with single rising bubbles by experimental and numerical approaches (as extensively reviewed by Besagni et al. (Besagni et al., 2016a; Besagni and Inzoli, 2016a)) and a correlation valid for swarm of rising bubbles would be interesting. The authors have proposed a first correlation for swarm of rising bubbles in an annular gap bubble column (Besagni and Inzoli, 2016a), which has been extended in a subsequent study (Besagni et al., 2016a). In particular, the correlation proposed by Besagni et al. (Besagni et al., 2016a) was used in statistical 


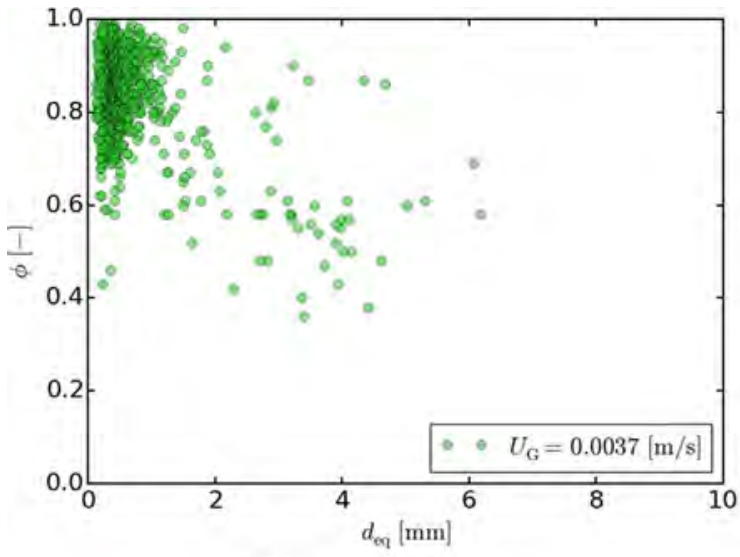

(a) $U_{G}=0.0037 \mathrm{~m} / \mathrm{s}$

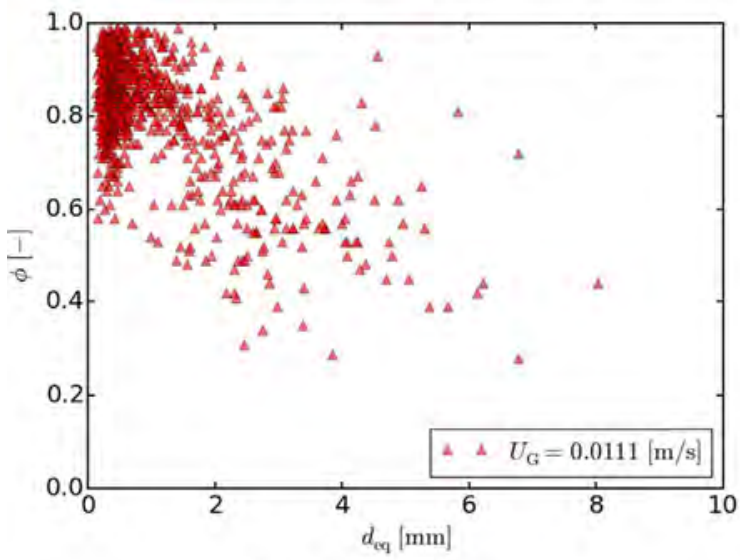

(c) $U_{G}=0.0111 \mathrm{~m} / \mathrm{s}$

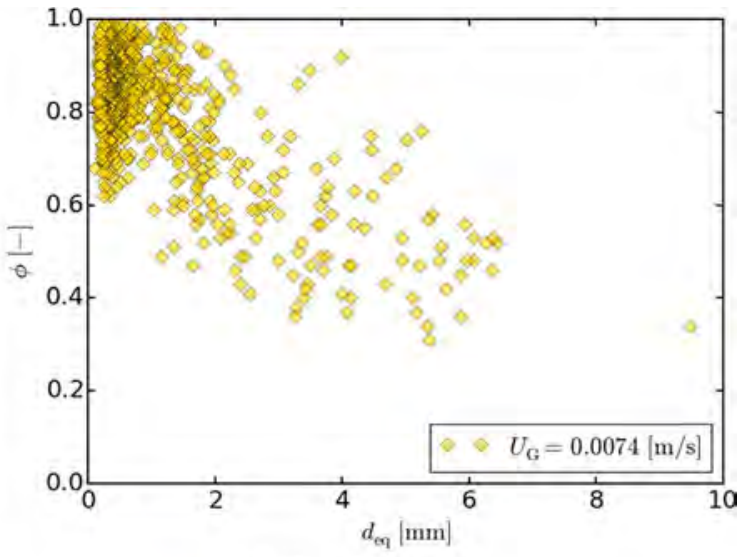

(b) $U_{G}=0.0074 \mathrm{~m} / \mathrm{s}$

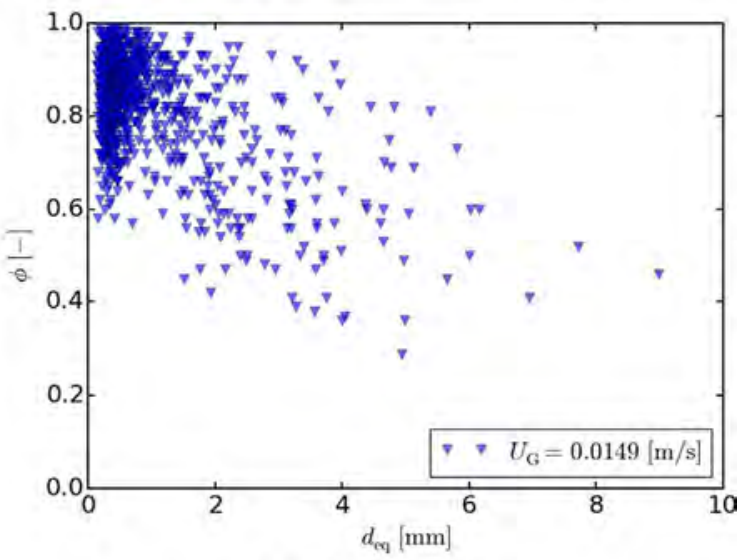

(d) $U_{G}=0.0149 \mathrm{~m} / \mathrm{s}$

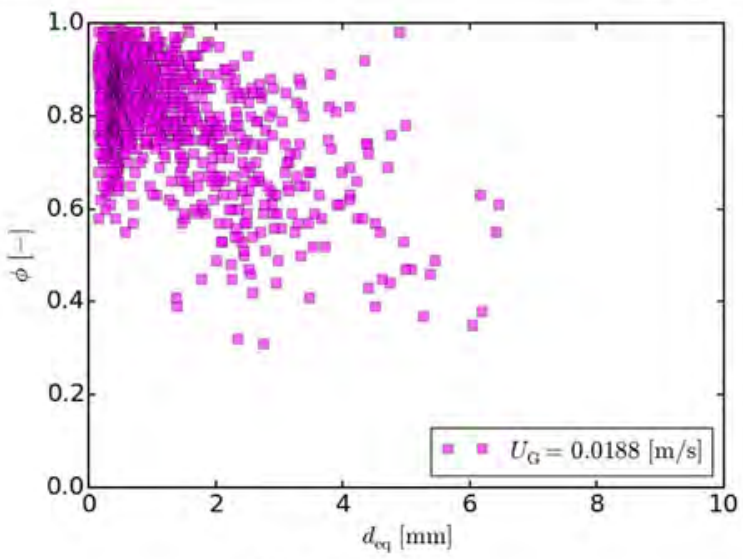

(e) $U_{G}=0.0188 \mathrm{~m} / \mathrm{s}$

Fig. 17. Relationship between the aspect ratio and the equivalent diameter $-c_{M E G}=5 \%-\log _{10}(M o)=-10.5202$ : influence of $U_{G}$.

algorithms to convert the chord length distributions obtained from the optical probe into bubble size distributions. This is of interest for the researchers working with bubble columns at high temperature and pressure, who wants to obtain bubble size distributions using optical probes. The goal of this section is to extend the previous correlation to other systems at different Mo numbers (please note that part of the method described in by Besagni et al. (Besagni et al., 2016a) has been repeated for the sake of clarity).

The experimental data obtained have been used and processed by the procedure described by Besagni et al. (Besagni et al., 2016a). It is worth noting that the aspect ratio data are scattered broadly; therefore, the data have been grouped into classes of equivalent diameters: each class is represented by the average aspect ratio of the bubbles belonging to that class. The validity of this approach was discussed in our previous papers (Besagni et al., 2016a; Besagni and Inzoli, 2016a). The relationship between the bubble shapes, the size of the bubbles, the speed of the bubbles and the physical properties of the system may be written as function of the following parameters:

$\varphi=f\left(u_{b}, \sigma, d_{e q}, \mu_{L}, \mu_{G}, \rho_{L}, \Delta \rho_{G-L}, g\right)$

Applying the dimensional analysis, Eq. (22) has been derived from Eq. (21): 


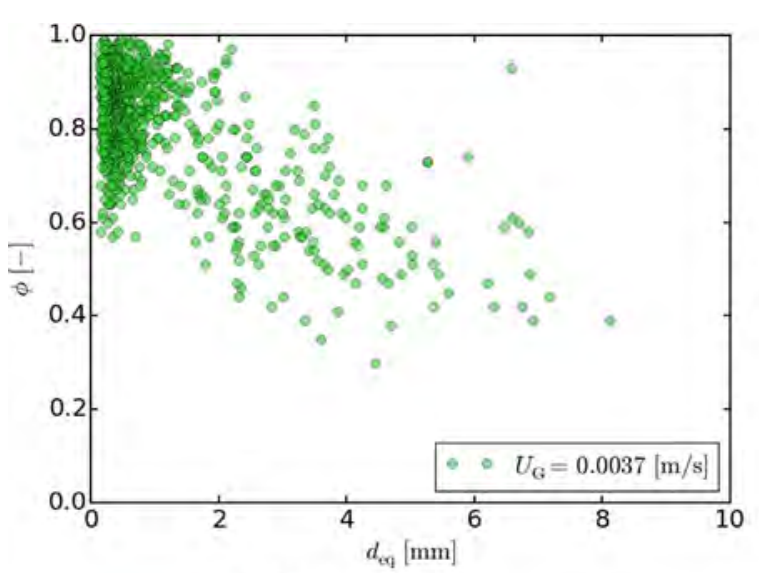

(a) $U_{G}=0.0037 \mathrm{~m} / \mathrm{s}$

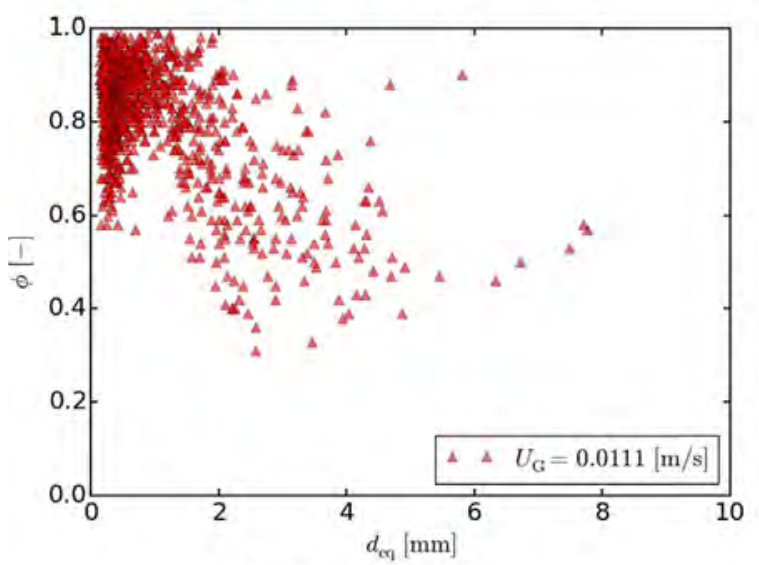

(c) $U_{G}=0.0111 \mathrm{~m} / \mathrm{s}$

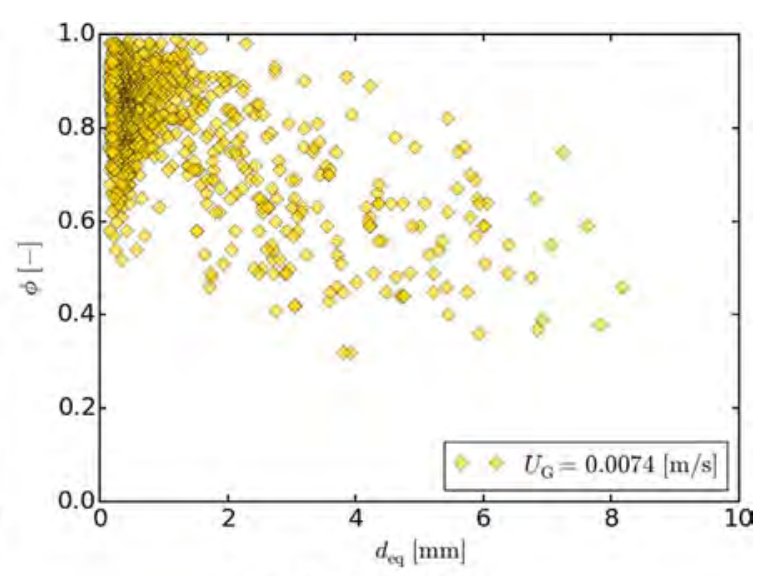

(b) $U_{G}=0.0074 \mathrm{~m} / \mathrm{s}$

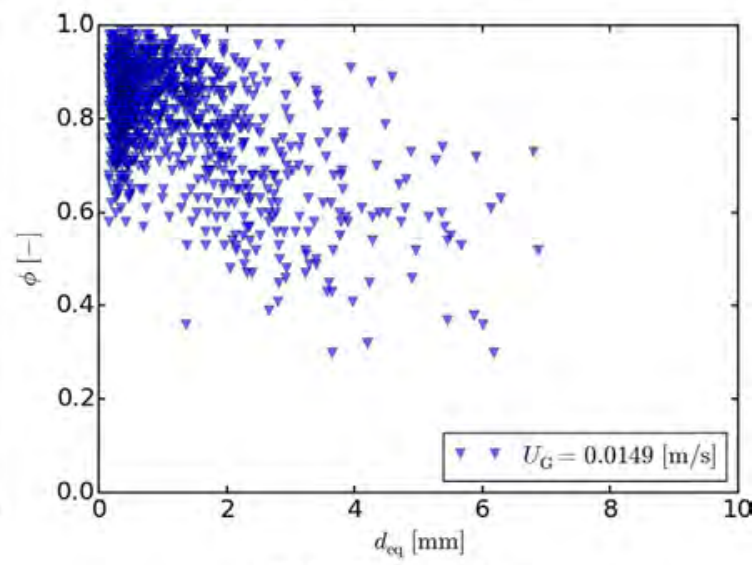

(d) $U_{G}=0.0149 \mathrm{~m} / \mathrm{s}$

Fig. 18. Relationship between the aspect ratio and the equivalent diameter $-c_{M E G}=8 \%-\log _{10}(M o)=-10.3708$ : influence of $U_{G}$.

$$
\begin{aligned}
\varphi & =f\left(\frac{d_{e q} u_{b}^{2} \rho_{L}}{\sigma}, \frac{d_{e q} u_{b} \rho_{L}}{\mu_{L}}, \frac{g \Delta \rho d_{e q}^{2}}{\sigma}, \frac{u_{b}^{2}}{g d_{e q}}, \frac{\mu_{L}}{\mu_{G}}\right) \\
& =f\left(W e, R e, E o, F r, N_{\mu_{r}}\right)
\end{aligned}
$$

The use of multiple dimensionless groups did not provide a benefit, and a single non-dimensional parameter can approximate the system: in the present work, $E o$ has been selected. We have also performed a more detailed statistical description of the experimental data (e.g. by using PDF of aspect ratios for every bubble sizes) and we have observed that it does not provide considerable benefits (Besagni et al., 2016a).

In order to propose a new correlation, firstly, a scheme of correlation must be selected for the function $f$, and the following has been applied:

$\varphi=f\left(W e, R e, E o, F r, N_{\mu_{r}}\right) \rightarrow f(E o)=k_{0} E o^{k_{1}}=k_{0}\left(\frac{g \Delta \rho d_{e q}^{2}}{\sigma}\right)^{k_{1}}=k_{2} d_{e q} k_{3}$

Given the mathematical structure of the algorithm described in Besagni et al. (Besagni et al., 2016a), a correlation between the bubble aspect ratio and the bubble major semi-axis (a) has been used:

$\varphi \underset{a=2 d_{e q} \varphi^{-\frac{1}{3}}}{\longrightarrow} k_{2} \cdot\left(\frac{1}{2} a \varphi^{\frac{1}{3}}\right)^{k_{3}}=k_{4} \bullet a^{k_{5}}$

At this point, two issues should be considered: (i) the smaller the bubble, the higher the sampling error (Section 2.3 and ref. (Besagni and Inzoli, 2016a, 2016b)); (ii) there are not enough bubbles having high $d_{e q}$. In order to deal with these issues, the following considerations have been accounted:
- Bubbles having $a<0.55 \mathrm{~mm}\left(\boldsymbol{d}_{\mathrm{eq}}<1 \mathrm{~mm}\right)$. These data have been not considered and, instead, the bubbles have been approximated as spherical bubbles, as described by the Bozzano-Dente (Bozzano and Dente, 2001) correlation;

- Bubbles having $a>5.5 \mathrm{~mm}\left(d_{e q}>8 \mathrm{~mm}\right)$. The BozzanoDente (Bozzano and Dente, 2001) correlation has been applied.

Therefore, the coefficients $k_{4}$ and $k_{5}$ in Eq. (24) have been computed by using a statistical regression of the experimental data (Fig. 8) in the region $0.55<a<5.5 \mathrm{~mm}$. A parabolic function (which is able to approximate the Bozzano-Dente (Bozzano and Dente, 2001) correlation) has been used in the range $a<0.55 \mathrm{~mm}$. An exponential function asymptotic to the Bozzano-Dente (Bozzano and Dente, 2001) correlation has been used in the range $a>5.5 \mathrm{~mm}$. The resulting correlation reads as follows (and its coefficients, for the different aqueous solutions of MES, are listed in Table $3^{2}$ ):

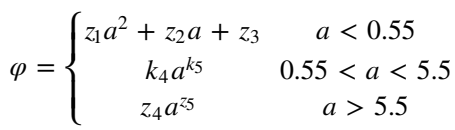

The comparison between our correlation and the Wellek et al. (Wellek et al., 1966) and the Bozzano-Dente (Bozzano and Dente, 2001) correlations has been detailed in (Besagni et al., 2016a) and

\footnotetext{
${ }^{2}$ Please note that there was a typo in the values reported in Table 1 of Besagni et al. (2016a). Estimation of bubble size distributions and shapes in two-phase bubble column using image analysis and optical probes. Flow Measurement and Instrumentation. and come of the coefficients for the air-water system was wrong. We are sorry for this mistake.
} 


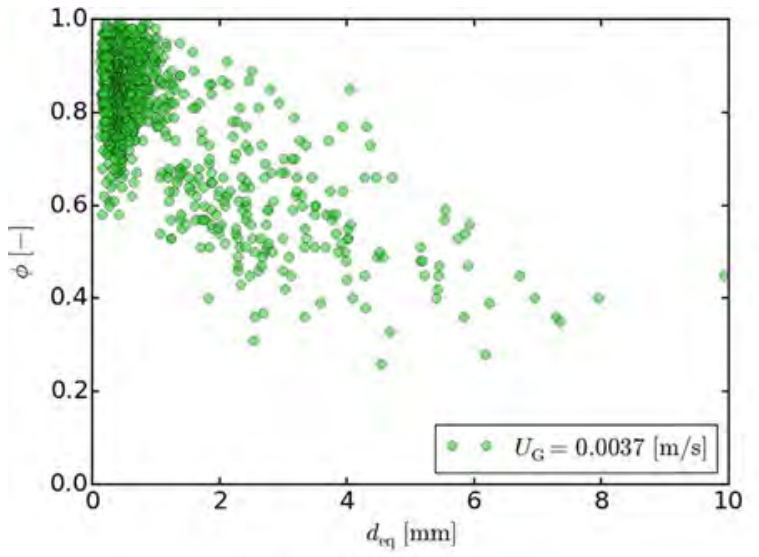

(a) $U_{G}=0.0037 \mathrm{~m} / \mathrm{s}$

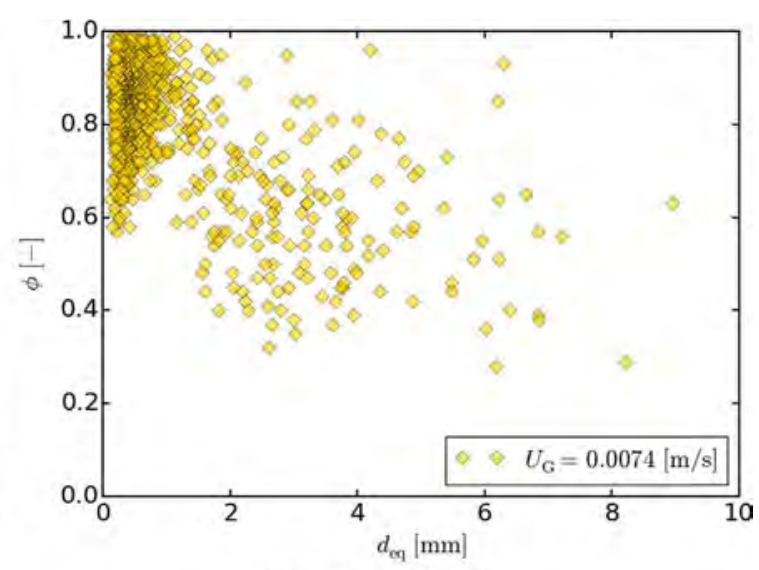

(b) $U_{G}=0.0074 \mathrm{~m} / \mathrm{s}$

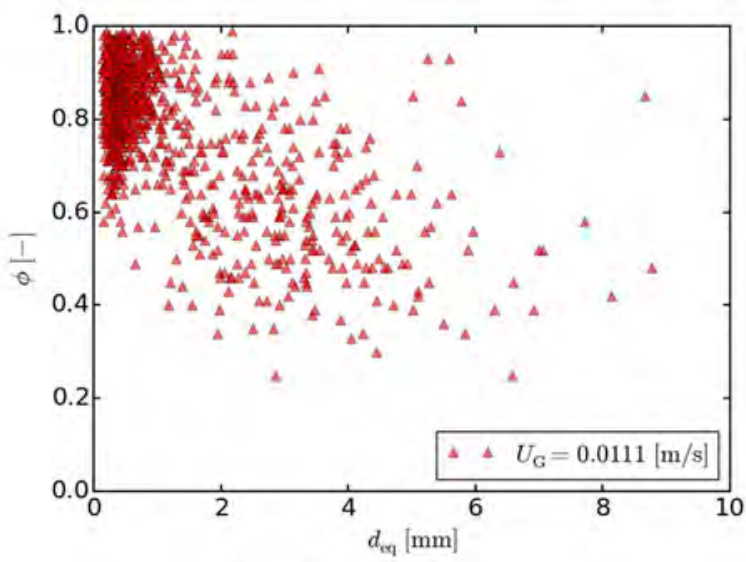

(c) $U_{G}=0.0111 \mathrm{~m} / \mathrm{s}$

Fig. 19. Relationship between the aspect ratio and the equivalent diameter $-c_{M E G}=10 \%-\log _{10}(M o)=-10.2703$ : influence of $U_{G}$.

similar conclusions apply here: correlations obtained for single bubbles/drops should be carefully evaluated before being applied to dense bubbly flows. It is worth noting that Eq. (25) has been obtained by considering the complete experimental dataset obtained for different gas velocities $\left(U_{G}\right.$ between $0.0037 \mathrm{~m} / \mathrm{s}$ and $\left.0.0188 \mathrm{~m} / \mathrm{s}\right)$. Applying the same procedure described above to smaller datasets, we observed that the relationship between $\varphi$ and the bubble size does not depend upon $U_{G}$. Therefore, given the above consideration, the conclusions listed in Besagni et al. (Besagni et al., 2016a; Besagni and Inzoli, 2016a) are considered general and apply to different systems (and not only airwater ones):

1. a correlation obtained considering all the bubbles sampled at different gas superficial velocities and in different sampling positions can be used without loss of generality;

2. the relationship between bubble sizes and shapes (in the homogeneous flow regime) mainly depends upon the system considered (i.e., the liquid phase properties) and not on the operating conditions;

The proposed correlations can be included in the statistical algorithm proposed by Besagni et al. (Besagni et al., 2016a), to convert the chord length distributions obtained from the optical probe into bubble size distributions. This is of practical interest for the engineers and/or researchers interesting to study BSDs in bubble columns working under relevant operating conditions (i.e. high pressure and temperature).

\subsubsection{Sauter mean diameter}

The results obtained for the BSDs can be discussed on the basis of the Sauter mean diameter $d_{23}$; indeed, the Sauter mean diameter is, simply, a statistical moment of the data, which is computed as:

$d_{23}=\frac{\sum_{i}^{N} n_{i} d_{e q, i}^{3}}{\sum_{i}^{N} n_{i} d_{e q, i}^{2}}$

where $d_{e q, i}$ and $n_{i}$ are the diameter and the number of bubbles of size class $i$, respectively, and $N$ is the number of classes used for the distribution.

Fig. 21a displays the Sauter mean diameter, $d_{23}$, for different $U_{G}$ values and MEG concentrations. A clear relation does not seem to exist, even if, for $U_{G}=0.149 \mathrm{~m} / \mathrm{s}, d_{23}$ always decreases and, then, starts increasing again. This behavior is hard to explain and further studies will be devoted to provide an insight. For example, Leonard et al. (Leonard et al., 2015) reviewed a large number of studies and stated that the influence of the gas superficial velocity over bubble size is far from being understood and controversial results have been published so far. Conversely, a relationship seems to exist between the gas velocity-averaged mean diameter $\left\langle d_{23}\right\rangle$ (evaluated by averaging $d_{23}$ for the different $U_{G}$ ) and the MEG concentration (Fig. 21b): < $d_{23}>$ decreases up to a minimum value $\left(c_{M E G}=5 \%\right)$ and then, starts increasing again up to $c_{M E G}=80 \%$. A decrease of $\left\langle d_{23}\right\rangle$ corresponds to an increase of the small bubbles peak in the BSDs, a stabilization of the homogeneous flow regime and an increase in the gas holdup, reaching its maximum at $c_{M E G}=5 \%$. Please note that the data presented in Fig. 21a and b at $U_{G}=0.0037 \mathrm{~m} / \mathrm{s}$ correspond to the flow visualizations in Fig. 4.

\subsubsection{Comparison with the previous literature}

For bubbles rising in an infinite medium, Clift et al. (Clift et al., 


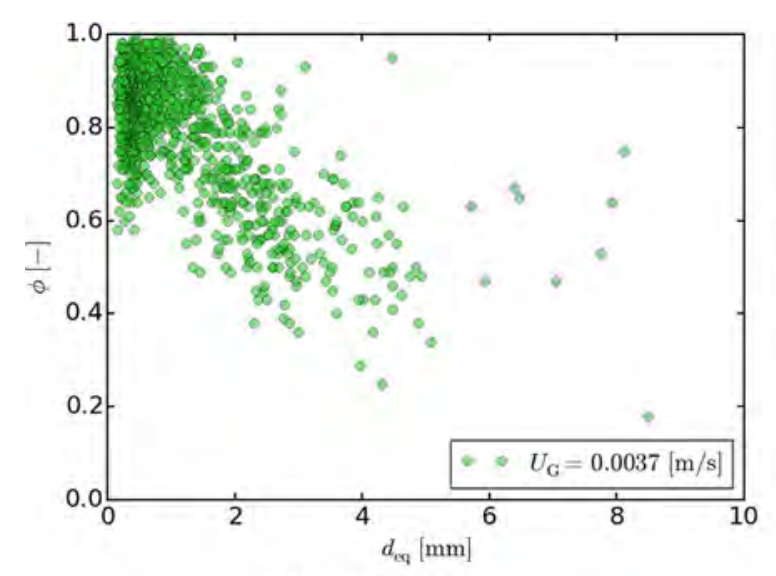

(a) $U_{G}=0.0037 \mathrm{~m} / \mathrm{s}$

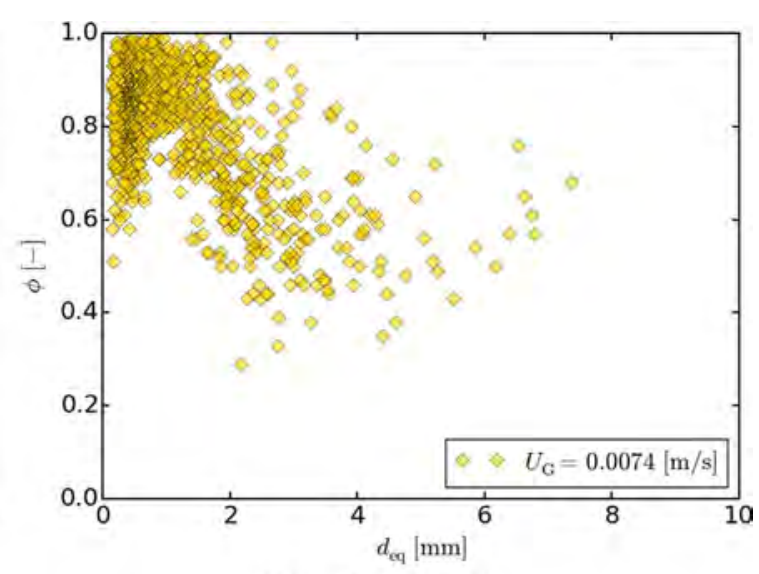

(b) $U_{G}=0.0074 \mathrm{~m} / \mathrm{s}$

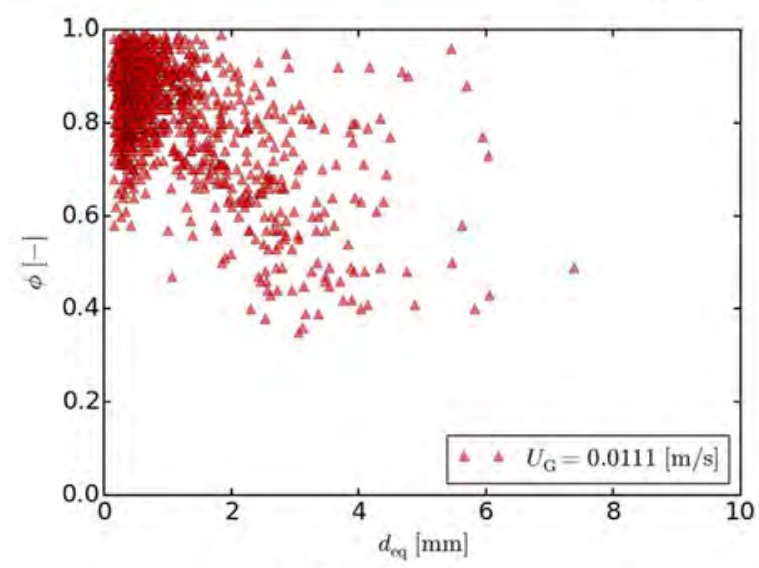

(c) $U_{G}=0.0111 \mathrm{~m} / \mathrm{s}$

Fig. 20. Relationship between the aspect ratio and the equivalent diameter $-c_{\mathrm{MEG}}=80 \%-\log _{10}(\mathrm{Mo})=-6.54542$ : influence of $\mathrm{U}_{\mathrm{G}}$.

1978) proposed a graphical correlation to determine the shapes of the bubbles in terms of $\mathrm{Eo}, \mathrm{Mo}$ and $\mathrm{Re}$. Mo is defined only by the properties of the fluids; for a constant $M o$, the shapes of the bubbles evolve from spherical to ellipsoidal to cap-shaped with increasing equivalent diameters. This is, of course, an approximation because this diagram was obtained for single rising bubbles rather than for swarms of rising bubbles. The velocity obtained is not the real bubble velocity but is the equivalent velocity of the bubble in the present system if placed in an infinite medium. Clift et al. (Clift et al., 1978) evaluated the influence of the wall on the rising bubbles according to the ratio $\lambda$ between the equivalent diameter of the bubble and the diameter of the system. For values of $\lambda$ less than 0.6 , the walls have little influence, and the rising bubbles can be considered as if they were rising in an infinite medium. The largest bubble detected in this analysis has an equivalent diameter of $17 \mathrm{~mm}$, and the hydraulic diameter of the column is equal to $0.24 \mathrm{~m}$. The maximum value of $\lambda$ is therefore 0.07 . Thus, the effect of the wall can be neglected as a first approximation. This diagram can be used to derive the terminal velocity $v_{b}$ for each bubble knowing that $E o$ and $M o, R e$ can be derived, and thus, so can the equivalent bubble terminal velocity $v_{b}$. This is, of course, an approximation because this diagram was obtained for single rising bubbles rather than for swarms of rising bubbles. The velocity obtained is not the real bubble velocity but is the equivalent velocity of the bubble in the present system if placed in an infinite medium. This approach was first proposed by Besagni and Inzoli (Besagni and Inzoli, 2016a). The data are represented in Fig. 22 for the air-water $\left(\log _{10}(M o)=-10.7724\right), c_{M E G}=10 \%\left(\log _{10}(M o)\right.$ $=-10.2703)$ and $c_{M E G}=80 \%\left(\log _{10}(M o)=-6.5454\right)$ systems. With regard to the shapes of the bubbles, the data obtained from the analysis of the images agree with the diagram by Clift et al. (Clift et al., 1978): the bubbles sampled in the air-water and $c_{M E G}=10 \%$ systems cover the spherical and ellipsoidal area. In contrast, some of the bubbles sampled in the $c_{M E G}=80 \%$ system cover the cap-shaped region (Fig. 23).

3.4. Relationship between BSDs and flow regime transition: "bubblescale" and "reactor-scale"

\subsubsection{Concept and main assumptions}

In the previous sections, a possible relationship between the bubble size distributions and the homogeneous flow regime stabilization/ destabilization has been proposed. In particular, the homogeneous flow regime stabilization/destabilization has been interpreted by analyzing the poly-dispersed nature of the BSDs with respect to the change in lift force direction. The main goal of this approach is to provide a first step towards understanding the underlying mechanisms of the bubble column stabilization/destabilization through a relatively simple approach based on the image analysis. Of course, this analysis has some main assumptions (which are matters for future studies). First, we have neglected the role of the non-lift forces (i.e., the drag, the turbulent dispersion and the virtual mass forces). In this respect, it is worth noting that the forces acting on a bubble have often been expressed in terms of the aspect ratio and depend on the bubble shape, as reviewed in the introduction of Aoyama et al. (Aoyama et al., 2016). For example, the virtual mass coefficient of a sphere is 0.5 , whereas for ellipsoidal bubbles it is given as a tensor (where the components are functions of the bubble aspect ratio). Second, we have considered a lift force model obtained for single rising bubbles: at the present state, models for bubble swarms are not fully developed, and are matters for ongoing studies and intensive research. Finally, we have assumed that a model for the lift force is able to account for all the cases investigate 


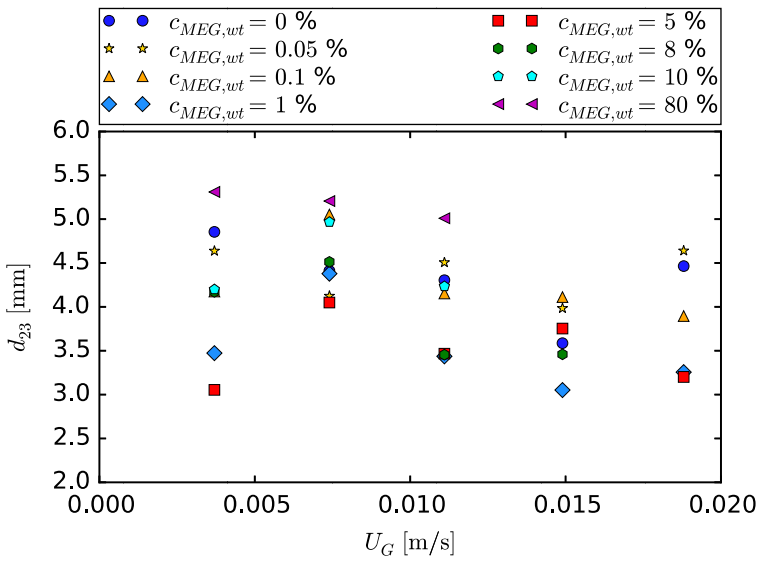

(a) Sauter mean diameter: influence of $U_{G}$ and MEG concentration

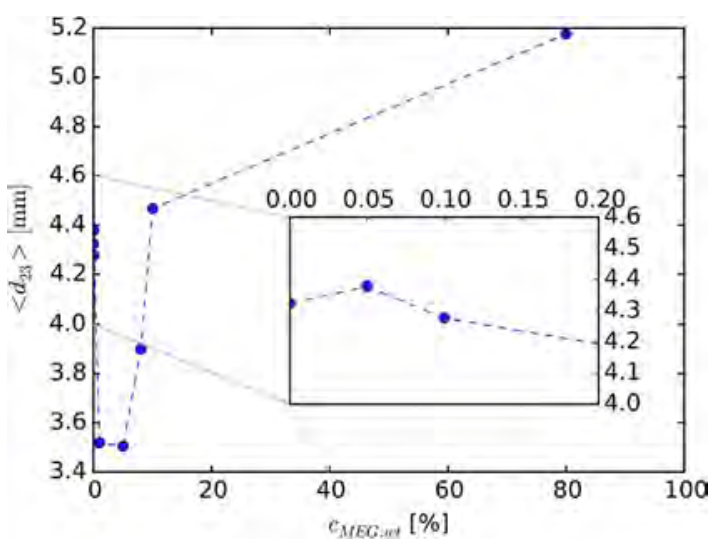

(b) Gas velocity-averaged $d_{23}$ : influence of MEG concentration

Fig. 21. Sauter mean diameter: influence of liquid phase properties and $U_{G}$.

Table 3

Coefficients of the aspect ratio correlation (Eq. (25)) for the system air-MEG aqueous solution.

\begin{tabular}{lllllllll}
\hline $\begin{array}{l}\mathbf{c}_{\mathbf{M E G}} \\
\text { [wt\%] }\end{array}$ & $\begin{array}{l}\mathbf{l o g}_{10} \\
\text { (Mo) [-] }\end{array}$ & $\mathbf{z}_{\mathbf{1}}$ & $\mathbf{z}_{\mathbf{2}}$ & $\mathbf{z}_{\mathbf{3}}$ & $\mathbf{k}_{\mathbf{4}}$ & $\mathbf{k}_{\mathbf{5}}$ & $\mathbf{z}_{\mathbf{4}}$ & $\mathbf{z}_{\mathbf{5}}$ \\
\hline 0 & -10.7724 & -0.657 & 0.001 & 1.00 & 0.690 & -0.251 & 0.750 & -0.300 \\
0.05 & -10.7697 & -0.570 & 0.001 & 1.00 & 0.691 & -0.300 & 0.670 & -0.280 \\
0.1 & -10.7676 & -0.550 & 0.001 & 1.00 & 0.701 & -0.294 & 0.692 & -0.280 \\
0.5 & -10.7466 & -0.666 & 0.001 & 1.00 & 0.674 & -0.308 & 0.666 & -0.300 \\
1 & -10.721 & -0.560 & 0.001 & 1.00 & 0.689 & -0.321 & 0.667 & -0.300 \\
5 & -10.5202 & -0.420 & 0.001 & 1.00 & 0.730 & -0.295 & 0.735 & -0.300 \\
8 & -10.3708 & -0.340 & 0.001 & 1.00 & 0.744 & -0.300 & 0.745 & -0.300 \\
10 & -10.2703 & -0.625 & 0.001 & 1.00 & 0.693 & -0.385 & 0.710 & -0.300 \\
80 & -6.54542 & -0.340 & 0.001 & 1.00 & 0.730 & -0.237 & 0.703 & -0.300 \\
\hline
\end{tabular}

(this assumption is further discussed in the following).

\subsubsection{The lift force approach}

The lift force acts perpendicularly to the rising bubbles and arises from the net effect of pressure and stress acting on the bubble surface. The lift force reads as:

$\vec{F}_{L i f t, G}=-C_{L} \alpha_{G} \rho_{L}\left(\vec{u}_{G}-\vec{u}_{L}\right) \times\left(\nabla \times \vec{u}_{L}\right)$

Where $C_{L}$ is the lift coefficient, $\rho_{L}$ is the liquid density, $\alpha_{G}$ is the local gas fraction and $\vec{u}_{G}-\vec{u}_{L}$ is the relative velocity between the gas and the liquid phases. The sign of the lift coefficient is positive for "small bubbles" and is negative for "large bubbles". The change of the sign of the lift coefficient defines a critical bubble diameter, $d_{c r}$. For this reason, the direction of the lift force depends upon the bubble size and shape. For "small bubbles", the lift force acts in the direction of decreasing liquid velocity (i.e., in case of a batch or co-current mode in

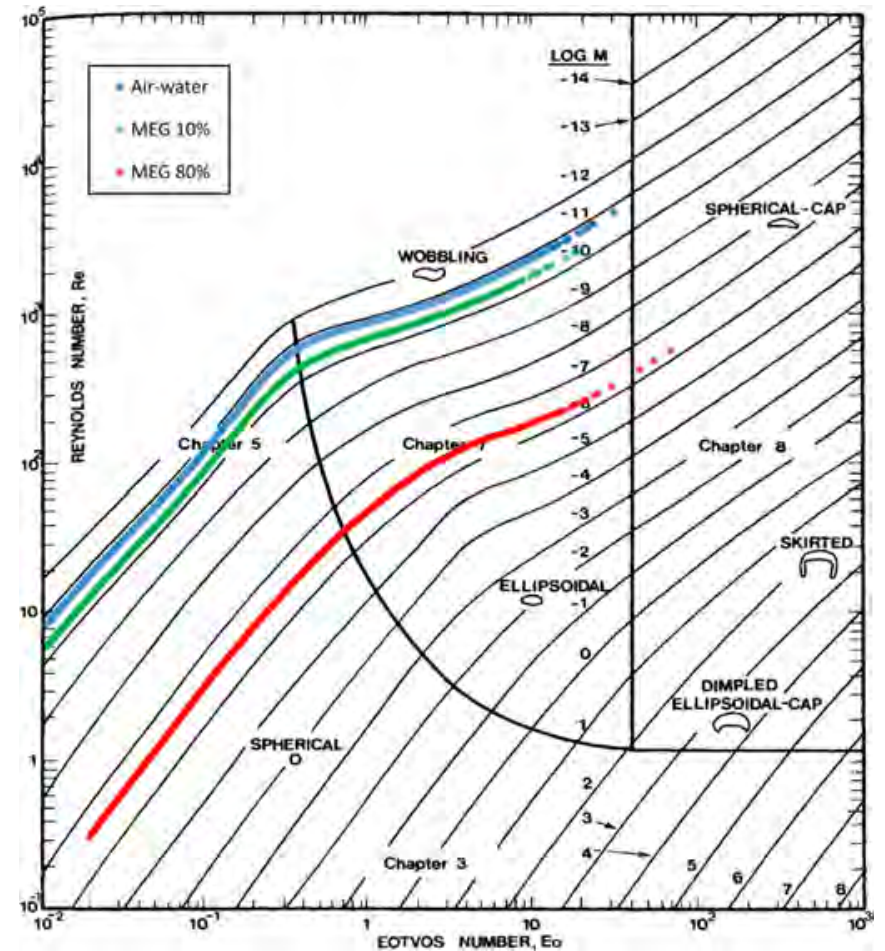

Fig. 22. Comparison between experimental data and the Clift diagram.

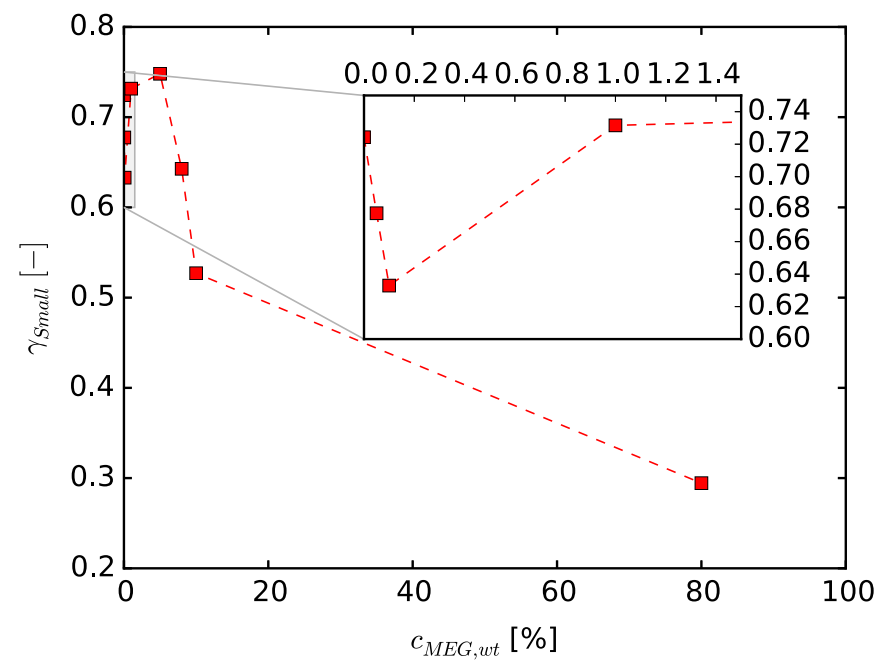

Fig. 23. Small bubble volume fraction: Influence of MEG concentration.

the direction toward the pipe wall), while for "large bubbles" it changes direction (a force that can be assimilated to the lift force, which tends to push large and deformed bubbles towards the center of the column (Lucas et al., 2005; Tomiyama et al., 2002)). In the counter-current mode, the behavior (supposing that the maximum downward velocity of the liquid is in the center of the column) is the opposite: the "small bubbles" migrate toward the center of the column. This is also confirmed by the DNS studies by Lu et al. (Lu et al., 2006), Lu and Tryggvason (Lu and Tryggvason, 2007, 2013) and Santarelli and Fröhlich (Santarelli and Fröhlich, 2016). To account for this dependency, the lift coefficient according to Tomiyama et al. (Tomiyama et al., 2002) is given as:

$C_{L}=\left\{\begin{array}{cc}\min \left[0.288 \tanh \left(0.121 \mathrm{Re}_{b}\right), f\left(E o_{\perp}\right)\right] & E o_{\perp} \leq 4 \\ f\left(E o_{\perp}\right) & 4<E o_{\perp} \leq 10 \\ -0.27 & 10<E o_{\perp}\end{array}\right.$

Where $f\left(E o_{\perp}\right)$ reads as follows: 
$f\left(E o_{\perp}\right)=0.00105 E o_{\perp}^{3}-0.0159 E o_{\perp}^{2}-0.0204 E o_{\perp}+0.474$

And $R e_{b}$ reads as follows:

$\operatorname{Re}_{b}=\rho_{L}\left|\vec{u}_{G}-\vec{u}_{L}\right| d_{b} / \mu_{L}$

And $E o_{\perp}$ is the Eötvös number considering the maximum horizontal dimension of the bubble $d_{\perp}$ given by the empirical correlation for the bubble shape by Wellek et al. (Wellek et al., 1966):

$E o_{\perp}=g\left|\rho_{L}-\rho_{G}\right| d_{\perp}^{2} / \sigma$

$d_{\perp}=d_{b}\left(1+0.163 E o^{0.757}\right)^{1 / 3}$

The experimental conditions, on which Eq. (28) is based, were limited to the range of $-5.5 \leq \log _{10}(M o) \leq-2.8,1.39 \leq$ Eo $\leq 5.74$ and values of the Reynolds number based on bubble diameter and shear rate of $0 \leq R e \leq 10$. The water-air system at normal conditions has higher $\log _{10}(\mathrm{Mo})$, but good results have nevertheless been reported for this case (Lucas and Tomiyama, 2011) and, recently, verified in the dissertation of Ziegenhein (Ziegenhein, 2016). Therefore, to the authors' opinion this formulation of the lift coefficient is well suited for the present case (Table $1: \log _{10}(M o)$ is in the range of -10.7724 and -6.5454). Of course, this formulation of the lift force is strictly valid for single bubble and its application to swarm of rising bubbles, despite very common and widely used (i.e. ref. (Ziegenhein et al., 2015)), and is a matter of future and intensive research and verification. It is worth noting that the use of Eq. (32) within the lift force model may be questionable and future studies should propose other formulations (i.e., our aspect ratio correlation, Eq. (25)).

Given the critical diameter $d_{c r}$-corresponding to the change of the sign of the lift coefficient, Eq. (28)-two bubble groups are defined as follows:

- "small bubbles" $\left(d<d_{c r}\right)$, with a positive lift coefficient;

- "large bubbles" $\left(d>d_{c r}\right)$, with a negative lift coefficient.

For the air-water system at ambient conditions, the bubble diameter at which the change in sign occurs is $5.8 \mathrm{~mm}$. The critical diameter $d_{c r}$ is the same as for the air-water case for MEG concentrations below $1 \%$, and then it moves to $5.7 \mathrm{~mm}, 5.6 \mathrm{~mm}$ and $4.6 \mathrm{~mm}$, respectively, for MEG mass concentrations of $c_{M E G}=5 \%, 10 \%$ and $80 \%$. The volume fraction contribution, $\gamma$, for the small and large bubble groups is computed as:

$\gamma_{i}=\frac{\sum_{i=1}^{M} n_{i} d_{i}^{3}}{\sum_{i=1}^{N} n_{i} d_{i}^{3}}$

\subsubsection{Discussion of the results and outlooks}

Fig. 23 displays the volume fraction of the small bubbles, $\gamma_{\text {Small }}$, depending on MEG concentration. $\gamma_{\text {Small }}$ increases to $74.8 \%\left(c_{M E G}\right.$ $=5 \%)$ and, then, decreases to $29.4 \%\left(c_{M E G}=80 \%\right)$. The decrease in $\gamma_{\text {small }}$ is caused by:

(1) the decrease in the critical diameter, $d_{c r}$;

(2) the presence of larger bubbles.

This result explains the relationship between the BSDs and the homogeneous flow regime stabilization/destabilization. Indeed, the "small bubbles" stabilize the homogeneous flow regime due to the lift force effect, as discussed by Lucas et al. (Lucas et al., 2006, 2005) (i.e., in this situation no larger vorticities are expected). Conversely, the "large bubbles" migrate toward the center of the pipe destabilizing the homogeneous flow regime. It is probable that the "large bubbles" migrate towards the center, thus creating the "coalescence-induced" structure (observed in (Besagni and Inzoli, 2016b)). According to Lucas et al. (Lucas et al., 2006), the critical value for the small bubble volume fraction to stabilize the homogeneous flow regime is $\gamma_{\text {Small, }}$ Cr $=45 \%$. This is in agreement with our experimental observations: the homogeneous flow regime is destabilized, compared with the air-water case, when $\gamma_{\text {Small }}=29.4 \%\left(c_{M E G}=80 \%\right)$. It is worth noting that the image analysis has considered only the bubbles near the wall: the overall "small bubble" contribution can be considerably different from the one illustrated in Fig. 23. Nevertheless, this analysis provides insight and reasonable explanations concerning the mechanisms behind the homogeneous flow regime stabilization/destabilization.

It should not be neglected that this analysis was performed under different assumptions and by applying the Tomiyama et al. (Tomiyama et al., 2002) lift force, which was obtained for single rising bubbles. Future studies will aim to improve and extend this analysis, by overcoming the assumptions listed before (of course, some of these assumptions are related to the state-of-the art in multiphase flow). However, to the authors' opinion, the results are promising and seems to provide insight into the mechanisms behind the bubble column homogeneous flow regime stabilization/destabilization.

\section{Conclusions, outcomes and future studies}

\subsection{Conclusions and outcomes}

This work experimentally investigates the dual effect of viscosity on the gas holdup, the flow regime transition, and the bubble size distributions in a large-diameter and large-scale bubble column. Air was used as the dispersed phase and various solutions of watermonoethylene glycol of different concentrations were employed as the liquid phase. To the authors' best knowledge, this is the first study concerning the dual effect of viscosity on gas holdup, flow regime transition and BSD in a large-diameter and large-scale bubble column.

The results suggest that the addition of monoethylene glycol to water changes the bubble interface properties (related to the coalescence suppression/enhancement mechanisms), which modifies the bubble size distribution and shapes, thus stabilizing/destabilizing the homogeneous flow regime and, ultimately, increasing/decreasing the gas holdup. Low viscosities are characterized by a larger number of small bubbles, which stabilizes the homogeneous flow regime, thus increasing the gas holdup. In contrast, moderate/high viscosities are characterized by larger bubbles, which destabilize the homogeneous flow regime and, thus, decrease the gas holdup. In particular, our results suggest that the stabilizing/destabilizing effect induced by the BSDs is related to the lift force and we have proposed a simple approach to explain this relationship. Large bubbles, having a negative lift coefficient, move toward the center of the pipes, thus promoting "coalescence-induced" bubbles and the flow regime transition. In contrast, small bubbles stabilize the homogeneous flow regime. It is worth noting that the observed relations between gas holdup, flow regime transition and BSDs are in agreement with a previous study proposed by the authors concerning an aqueous solution of ethanol.

In addition, based on visualizations, a correlation between bubble size and aspect ratio has been proposed. Taking into account our previous studies, it is concluded that the proposed correlation does not depend on the gas velocities and sampling position, suggesting that the relationship between bubble sizes and shapes (in the homogeneous flow regime) mainly depends upon the system considered and not on the operating conditions. The proposed correlations can be included in the statistical algorithm proposed by Besagni et al. (Besagni et al., 2016a) to convert the chord length distributions obtained from the optical probe into bubble size distributions. This is of practical interest for the engineers and/or researchers interesting to study BSDs in bubble columns working under relevant operating conditions (i.e. high pressure and temperature).

In conclusion, the experimental results are of practical interest in the design of bubble columns. Indeed, the present bubble column was built taking into account the well-known scale-up criteria and this may 
suggest that the present phenomena may also apply in industrial-scale bubble columns. Moreover, the results presented in this paper are particularly useful for the validation of numerical models.

\subsection{Future studies}

Other researchers have reported a dual effect on the gas holdup in other binary systems, when using active compounds. Further studies should be devoted to understand the common hydrodynamic properties of viscous and active compound systems, and to find a comprehensive theory/a common physical model to describe all the dual effects observed: (i) the dual effect of viscosity; (ii) the dual effect of organic compounds; and (iii) the dual effect of inorganic compounds. Considering the results obtained within this study, it is reasonable to assume that all the dual effects can be described by a unique model considering the effects at the bubble interface. In addition, future studies should be devoted to study the evolution of the BSDs in the axial and radial direction of the bubble column, to provide insights in the coalescence and break up phenomena.

\section{Acknowledgements}

This work was possible because of the contribution of many people. The authors thank Medea Carrara for the original implementation of the image analysis methods. The authors are grateful to Pietro Brazzale and Alberto Fiocca for their assistance in the experimental investigations and for sampling the bubbles. The authors would like to thank Dr. Dirk Lucas for all the discussion concerning the role of the lift force. The authors are, also, indebted to Prof. Paolo di Marco for the suggestions concerning the bubble shapes and sizes. The authors would like to thank the anonymous reviewers for their valuable comments. Finally, authors would like to thank Professor N.G. Deen for handling the paper. The authors wish to express their thanks to Itelcond srl and to Ing. Luca Primavesi, in particular, for having supplied monoethylene glycol for the experimental campaign.

\section{References}

Akita, K., Yoshida, F., 1973. Gas holdup and volumetric mass transfer coefficient in bubble columns. Effects of liquid properties. Ind. Eng. Chem. Process Des. Dev. 12, $76-80$.

Aloufi, F.M., 2011. An investigation of gas void fraction and transition conditions for two-phase flow in an annular gap bubble column. Loughborough University.

Andrew, S., 1960. Frothing in two-component liquid mixtures, Proceedings of the International Symposium on Distillation. PA Rottenburg, 73-78.

Aoyama, S., Hayashi, K., Hosokawa, S., Tomiyama, A., 2016. Shapes of ellipsoidal bubbles in infinite stagnant liquids. Int. J. Multiph. Flow. 79, 23-30.

Bach, H.F., Pilhofer, T., 1978. Variation of gas holdup in bubble columns with physical properties of liquids and operating parameters of columns. Ger. Chem. Eng. 1, $270-275$.

Besagni, G., 2016. Bubble column fluid dynamics: experimental and numerical investigations (PhD Thesis). Politecnico di Milano.

Besagni, G., Brazzale, P., Fiocca, A., Inzoli, F., 2016a. Estimation of bubble size distributions and shapes in two-phase bubble column using image analysis and optical probes. (In Press)Flow. Meas. Instrum..

Besagni, G., Guédon, G., Inzoli, F., 2014. Experimental investigation of counter current air-water flow in a large diameter vertical pipe with inners. J. Phys.: Conf. Ser. 547, 012024.

Besagni, G., Guédon, G.R., Inzoli, F., 2016b. Annular gap bubble column: experimental investigation and computational fluid dynamics modeling. J. Fluids Eng. 138, 011302.

Besagni, G., Inzoli, F., 2015. Influence of electrolyte concentration on holdup, flow regime transition and local flow properties in a large scale bubble column. J. Phys.: Conf. Ser., 655

Besagni, G., Inzoli, F., 2016a. Bubble size distributions and shapes in annular gap Bubble column. Exp. Therm. Fluid Sci. 4, 27-48.

Besagni, G., Inzoli, F., 2016b. Comprehensive experimental investigation of countercurrent bubble column hydrodynamics: holdup, flow regime transition, bubble size distributions and local flow properties. Chem. Eng. Sci. 146, 259-290.

Besagni, G., Inzoli, F., 2016c. Influence of internals on counter-current bubble column hydrodynamics: holdup, flow regime transition and local flow properties. Chem. Eng. Sci. 145, 162-180.

Besagni, G., Inzoli, F., De Guido, G., Pellegrini, L.A., 2016c. Experimental investigation on the influence of ethanol on bubble column hydrodynamics. Chem. Eng. Res. Des.
$112,1-15$.

Bhaga, D., Pruden, B.B., Weber, M.E., 1971. Gas holdup in a bubble column containing organic liquid mixtures. Can. J. Chem. Eng. 49, 417-420.

Bian, Y., Dong, F., Zhang, W., Wang, H., Tan, C., Zhang, Z., 2013. 3D reconstruction of single rising bubble in water using digital image processing and characteristic matrix. Particuology 11, 170-183.

Bozzano, G., Dente, M., 2001. Shape and terminal velocity of single bubble motion: a novel approach. Comput. Chem. Eng. 25, 571-576.

Brooks, C.S., Paranjape, S.S., Ozar, B., Hibiki, T., Ishii, M., 2012. Two-group drift-flux model for closure of the modified Two-fluid model. Int. J. Heat. Fluid Flow. 37 , 196-208.

Celata, G.P., Cumo, M., D'Annibale, F., Tomiyama, A., 2004. The wake effect on bubble rising velocity in one-component systems. Int. J. Multiph. Flow. 30, 939-961.

Celata, G.P., Cumo, M., D’Annibale, F., Di Marco, P., Tomiyama, A., Zovini, C., 2006. Effect of gas injection mode and purity of liquid on bubble rising in two-component systems. Exp. Therm. Fluid Sci. 31, 37-53.

Celata, G.P., D’Annibale, F., Di Marco, P., Memoli, G., Tomiyama, A., 2007. Measurements of rising velocity of a small bubble in a stagnant fluid in one- and twocomponent systems. Exp. Therm. Fluid Sci. 31, 609-623.

Churchill, S., Usagi, R., 1972. A general expression for the correlation of rates of transfer and other phenomena. AIChE J. 18, 1121-1128.

Clift, R., Grace, J.R., Weber, M.E., 1978. Bubbles, drops, and particles, New York.

Craig, V.S.J., Ninham, B.W., Pashley, R.M., 1993. The effect of electrolytes on bubble coalescence in water. J. Phys. Chem. 97, 10192-10197.

De Guido, G., Besagni, G., Inzoli, F., Pellegrini, L.A., 2016.New gas holdup data in large counter-current bubble columns, ICMF-2016 - In: Proceedings of the 9th International Conference on Multiphase Flow, Florence, Italy.

Deckwer, W.-D., Field, R.W., 1992. Bubble column reactors. Wiley, New York.

Deckwer, W.D., 1992. Bubble column reactors. John Wiley \& Sons Ltd, Baffins Lane, Chichester.

Dimakopoulos, Y., Pavlidis, M., Tsamopoulos, J., 2013. Steady bubble rise in HerschelBulkley fluids and comparison of predictions via the Augmented Lagrangian Method with those via the Papanastasiou model. J. Non-Newton. Fluid Mech. 200, 34-51.

Eissa, S.H., Schügerl, K., 1975. Holdup and backmixing investigations in cocurrent and countercurrent bubble columns. Chem. Eng. Sci. 30, 1251-1256.

Ellingsen, K., Risso, F., 2001. On the rise of an ellipsoidal bubble in water: oscillatory paths and liquid-induced velocity. J. Fluid Mech. 440, 235-268.

Ellis, J.E., Lloyd Jones, E., 1965. Vertical gas-liquid problems. Proc. Symp. Two-phase Flow., Exeter, B101-B140.

Firouzi, M., Howes, T., Nguyen, A.V., 2015. A quantitative review of the transition salt concentration for inhibiting bubble coalescence. Adv. Colloid Interface Sci. 222, $305-318$.

Fraggedakis, D., Pavlidis, M., Dimakopoulos, Y., Tsamopoulos, J., 2016. On the velocity discontinuity at a critical volume of a bubble rising in a viscoelastic fluid. J. Fluid Mech. 789, 310-346.

Godbole, S.P., Honath, M.F., Shah, Y.T., 1982. Holdup structure in highly viscous Newtonian and non-Newtonian liquids in bubble columns. Chem. Eng. Commun. 16, 119-134.

Gourich, B., Vial, C., Essadki, A.H., Allam, F., Belhaj Soulami, M., Ziyad, M., 2006. Identification of flow regimes and transition points in a bubble column through analysis of differential pressure signal-Influence of the coalescence behavior of the liquid phase. Chem. Eng. Process.: Process Intensif. 45, 214-223.

Gumulya, M., Joshi, J.B., Utikar, R.P., Evans, G.M., Pareek, V., 2016. Bubbles in viscous liquids: time dependent behaviour and wake characteristics. Chem. Eng. Sci. 144, 298-309.

Guo, K., Wang, T., Yang, G., Wang, J., 2016. Distinctly different bubble behaviors in a bubble column with pure liquids and alcohol solutions. J. Chem. Technol. Biotechnol., (n/a-n/a).

Hanselmann, W., Windhab, E., 1998. Flow characteristics and modelling of foam generation in a continuous rotor/stator mixer. J. Food Eng. 38, 393-405.

Hills, J.H., 1976. The operation of a bubble column at high throughputs: i. Gas holdup measurements. Chem. Eng. J. 12, 89-99.

Honkanen, M., Saarenrinne, P., Stoor, T., Niinimäki, J., 2005. Recognition of highly overlapping ellipse-like bubble images. Meas. Sci. Technol. 16, 1760-1770.

Hwang, S.-J., Cheng, Y.-L., 1997. Gas holdup and liquid velocity in three-phase internalloop airlift reactors. Chem. Eng. Sci. 52, 3949-3960.

Kaji, M., Sawai, T., Mori, K., Iguchi, M., 2001. Behaviours of bubble formation from a bottom porous nozzle bath, Proceedings of the Fifth ExHFT, Thessaloniki, Greece, pp. 1503-1508.

Karn, A., Ellis, C., Arndt, R., Hong, J., 2015. An integrative image measurement technique for dense bubbly flows with a wide size distribution. Chem. Eng. Sci. 122, 240-249.

Kazakis, N.A., Papadopoulos, I.D., Mouza, A.A., 2007. Bubble columns with fine pore sparger operating in the pseudo-homogeneous regime: gas hold up prediction and a criterion for the transition to the heterogeneous regime. Chem. Eng. Sci. 62, 3092-3103.

Kestin, J., Sokolov, M., Wakeham, W.A., 1978. Viscosity of liquid water in the range $-8^{\circ} \mathrm{C}$ to $150{ }^{\circ} \mathrm{C}$. J. Phys. Chem. Ref. Data 7, 941-948.

Khare, A.S., Joshi, J.B., 1990. Effect of fine particles on gas hold-up in three-phase sparged reactors. Chem. Eng. J. 44, 11-25.

Kitscha, J., Kocamustafaogullari, G., 1989. Breakup criteria for fluid particles. Int. J. Multiph. Flow. 15, 573-588.

Krishna, R., Urseanu, M.I., Dreher, A.J., 2000. Gas hold-up in bubble columns: influence of alcohol addition versus operation at elevated pressures. Chem. Eng. Process . Process Intensif. 39, 371-378.

Krishna, R., Wilkinson, P.M., Van Dierendonck, L.L.,, 1991. A model for gas holdup in 
bubble columns incorporating the influence of gas density on flow regime transitions. Chem. Eng. Sci. 46, 2491-2496.

Kuncová, G., Zahradník, J., 1995. Gas holdup and bubble frequency in a bubble column reactor containing viscous saccharose solutions. Chem. Eng. Process.: Process Intensif. 34, 25-34.

Lage, P.L.C., Espósito, R.O., 1999. Experimental determination of bubble size distributions in bubble columns: prediction of mean bubble diameter and gas hold up. Powder Technol. 101, 142-150.

Lau, Y.M., Deen, N.G., Kuipers, J.A.M., 2013a. Development of an image measurement technique for size distribution in dense bubbly flows. Chem. Eng. Sci. 94, 20-29.

Lau, Y.M., Sujatha, K.T., Gaeini, M., Deen, N.G., Kuipers, J.A.M., 2013b. Experimental study of the bubble size distribution in a pseudo-2D bubble column. Chem. Eng. Sci. 98, 203-211.

Leonard, C., Ferrasse, J.H., Boutin, O., Lefevre, S., Viand, A., 2015. Bubble column reactors for high pressures and high temperatures operation. Chem. Eng. Res. Des. 100, 391-421.

Lessard, R.R., Zieminski, S.A., 1971. Bubble coalescence and gas transfer in aqueous electrolytic solutions. Ind. Eng. Chem. Fundam. 10, 260-269.

Letzel, H.M., Schouten, J.C., van den Bleek, C.M., Krishna, R., 1997. Influence of elevated pressure on the stability of bubbly flows. Chem. Eng. Sci. 52, 3733-3739.

Liao, Y., Lucas, D., 2009. A literature review of theoretical models for drop and bubble breakup in turbulent dispersions. Chem. Eng. Sci. 64, 3389-3406.

Liao, Y., Lucas, D., 2010. A literature review on mechanisms and models for the coalescence process of fluid particles. Chem. Eng. Sci. 65, 2851-2864.

Lu, J., Biswas, S., Tryggvason, G., 2006. A DNS study of laminar bubbly flows in a vertical channel. Int. J. Multiph. Flow. 32, 643-660.

Lu, J., Tryggvason, G., 2007. Effect of bubble size in turbulent bubbly downflow in a vertical channel. Chem. Eng. Sci. 62, 3008-3018.

Lu, J., Tryggvason, G., 2013. Dynamics of nearly spherical bubbles in a turbulent channel upflow. J. Fluid Mech. 732, 166-189.

Lucas, D., Krepper, E., Prasser, H.M., 2003. Evolution of flow patterns, gas fraction profiles and bubble size distributions in gas-liquid flows in vertical tubes. Trans. Inst. Fluid-Flow. Mach. 112, 37-46.

Lucas, D., Krepper, E., Prasser, H.M., Manera, A., 2006. Investigations on the Stability of the Flow Characteristics in a Bubble Column. Chem. Eng. Technol. 29, 1066-1072.

Lucas, D., Prasser, H.M., Manera, A., 2005. Influence of the lift force on the stability of a bubble column. Chem. Eng. Sci. 60, 3609-3619.

Lucas, D., Rzehak, R., Krepper, E., Ziegenhein, T., Liao, Y., Kriebitzsch, S., Apanasevich, P., 2015. A strategy for the qualification of multi-fluid approacheds for nuclear reactor safety. Nuclear Engineering and Design.

Lucas, D., Tomiyama, A., 2011. On the role of the lateral lift force in poly-dispersed bubbly flows. Int. J. Multiph. Flow. 37, 1178-1190.

MEGlobalTM, 2015. Ethylene Glycol. Product Guide. Last accessed on October 17th, 2015.

Moore, D.W., 1959. The rise of a gas bubble in a viscous liquid. J. Fluid Mech. 6, $113-130$.

Mudde, R.F., Harteveld, W.K., van den Akker, H.E.A., 2009. Uniform flow in bubble columns. Ind. Eng. Chem. Res. 48, 148-158.

Nedeltchev, S., 2015. New methods for flow regime identification in bubble columns and fluidized beds. Chem. Eng. Sci. 137, 436-446.

Nedeltchev, S., Hampel, U., Schubert, M., 2016. Investigation of the radial effect on the transition velocities in a bubble column based on the modified Shannon entropy. Chem. Eng. Res. Des. 115 (Part B), 303-309.

Nedeltchev, S., Schubert, M., 2015. Statistical validation of the mixing length concept in bubble columns operated in the transition flow regime. J. Chem. Eng. Jpn. 48, $107-111$.

Nedeltchev, S., Shaikh, A., 2013. A new method for identification of the main transition velocities in multiphase reactors based on information entropy theory. Chem. Eng. Sci. 100, 2-14.

Nguyen, P.T., Hampton, M.A., Nguyen, A.V., Birkett, G.R., 2012. The influence of gas velocity, salt type and concentration on transition concentration for bubble coalescence inhibition and gas holdup. Chem. Eng. Res. Des. 90, 33-39.

Okawa, T., Tanaka, T., Kataoka, I., Mori, M., 2003. Temperature effect on single bubble rise characteristics in stagnant distilled water. Int. J. Heat. Mass Transf. 46, 903-913.

Olivieri, G., Elena Russo, M., Simeone, M., Marzocchella, A., Salatino, P., 2011. Effects of viscosity and relaxation time on the hydrodynamics of gas-liquid systems. Chem. Eng. Sci. 66, 3392-3399.

Orvalho, S., Ruzicka, M.C., Drahos, J., 2009. Bubble Column with Electrolytes: gas Holdup and Flow Regimes. Ind. Eng. Chem. Res. 48, 8237-8243.

Passos, A.D., Voulgaropoulos, V.P., Paras, S.V., Mouza, A.A., 2015. The effect of surfactant addition on the performance of a bubble column containing a nonNewtonian liquid. Chem. Eng. Res. Des. 95, 93-104.

Perry, R.H., Green, D.W., 2008. Perry's chemical engineers' handbook 7th ed.. McGrawHill, New York.

Philip, J., Proctor, J.M., Niranjan, K., Davidson, J.F., 1990. Gas hold-up and liquid circulation in internal loop reactors containing highly viscous newtonian and nonnewtonian liquids. Chem. Eng. Sci. 45, 651-664.

Rabha, S., Schubert, M., Hampel, U., 2014. Regime transition in viscous and pseudo viscous systems: a comparative study. AIChE J. 60, 3079-3090.
Rakoczy, R., Masiuk, S., 2009. Experimental study of bubble size distribution in a liquid column exposed to a rotating magnetic field. Chem. Eng. Process.: Process Intensif. 48, 1229-1240.

Reilly, I., Scott, D., Debruijn, T., MacIntyre, D., 1994. The role of gas phase momentum in determining gas holdup and hydrodynamic flow regimes in bubble column operations. Can. J. Chem. Eng. 72, 3-12.

Ribeiro, C.P., Jr, Mewes, D., 2007. The influence of electrolytes on gas hold-up and regime transition in bubble columns. Chem. Eng. Sci. 62, 4501-4509.

Richardson, J.F., Zaki, W.N., 1997. Sedimentation and fluidisation: Part I. Chem. Eng. Res. Des. 75 (Supplement), S82-S100.

Rollbusch, P., Bothe, M., Becker, M., Ludwig, M., Grünewald, M., Schlüter, M., Franke, R., 2015. Bubble columns operated under industrially relevant conditions - Current understanding of design parameters. Chem. Eng. Sci. 126, 660-678.

Rubia, M.D.L., García-Abuín, A., Gómez-Díaz, D., Navaza, J.M., 2010. Interfacial area and mass transfer in carbon dioxide absorption in TEA aqueous solutions in a bubble column reactor. Chem. Eng. Process.: Process Intensif. 49, 852-858.

Ruzicka, M.C., Drahoš, J., Mena, P.C., Teixeira, J.A., 2003. Effect of viscosity on homogeneous-heterogeneous flow regime transition in bubble columns. Chem. Eng. J. 96, 15-22.

Ruzicka, M.C., Vecer, M.M., Orvalho, S., Drahoš, J., 2008. Effect of surfactant on homogeneous regime stability in bubble column. Chem. Eng. Sci. 63, 951-967.

Ruzicka, M.C., Zahradník, J., Drahoš, J., Thomas, N.H., 2001. Homogeneousheterogeneous regime transition in Bubble columns. Chem. Eng. Sci. 56, 4609-4626.

Sahagian, D.L., Proussevitch, A.A., 1998. 3D particle size distributions from 2D observations: stereology for natural applications. J. Volcanol. Geotherm. Res. 84, $173-196$.

Santarelli, C., Fröhlich, J., 2016. Direct Numerical Simulations of spherical bubbles in vertical turbulent channel flow. Influence of bubble size and bidispersity. Int. J. Multiph. Flow. 81, 27-45.

Shah, Y.T., Joseph, S., Smith, D.N., Ruether, J.A., 1985. On the behavior of the gas phase in a bubble column with ethanol-water mixtures. Ind. Eng. Chem. Process Des. Dev. $24,1140-1148$.

Shah, Y.T., Kelkar, B.G., Godbole, S.P., Deckwer, W.D., 1982. Design parameters estimations for bubble column reactors. AIChE J. 28, 353-379.

Sharaf, S., Zednikova, M., Ruzicka, M.C., Azzopardi, B.J., 2015. Global and local hydrodynamics of bubble columns - Effect of gas distributor. Chem. Eng. J. 288, 489-504.

Sikorski, D., Tabuteau, H., de Bruyn, J.R., 2009. Motion and shape of bubbles rising through a yield-stress fluid. J. Non-Newton. Fluid Mech. 159, 10-16.

Sun, T., Teja, A.S., 2003. Density, viscosity, and thermal conductivity of aqueous ethylene, diethylene, and triethylene glycol mixtures between $290 \mathrm{~K}$ and $450 \mathrm{~K}$. J. Chem. Eng. Data 48, 198-202.

Syeda, S.R., Afacan, A., Chuang, K.T., 2002. Prediction of gas hold-up in a bubble column filled with pure and binary liquids. Can. J. Chem. Eng. 80, 44-50.

Tadaki, T., Maeda, S., 1961. On the shape and velocity of single air bubbles rising in various liquids. Kagaku Kogaku 25, 254-264.

Taylor, T.D., Acrivos, A., 1964. On the deformation and drag of a falling viscous drop at low Reynolds number. J. Fluid Mech. 18, 466-476.

Tomiyama, A., Tamai, H., Zun, I., Hosokawa, S., 2002. Transverse migration of single bubbles in simple shear flows. Chem. Eng. Sci. 57, 1849-1858.

Tsamopoulos, J., Dilmakopoulos, Y., Chatzidai, N., Karapetsas, G., Pavlidis, M., 2008 Steady bubble rise and deformation in Newtonian and viscoplastic fluids and conditions for bubble entrapment. J. Fluid Mech. 601, 123-164.

Urseanu, M.I., 2000. Scaling up bubble column reactors.

Vargaftik, N., Volkov, B., Voljak, L., 1983. International tables of the surface tension of water. J. Phys. Chem. Ref. Data 12, 817-820.

Wallis, G.B., 1969. One-dimensional two-phase flow, New York

Wellek, R.M., Agrawal, A.K., Skelland, A.H.P., 1966. Shape of liquid drops moving in liquid media. AIChE J. 12, 854-862.

Wilkinson, P.M., Spek, A.P., van Dierendonck, L.L., 1992. Design parameters estimation for scale-Up of high-pressure bubble columns. AIChE J. 38, 544-554.

Wongsuchoto, P., Charinpanitkul, T., Pavasant, P., 2003. Bubble size distribution and gas-liquid mass transfer in airlift contactors. Chem. Eng. J. 92, 81-90.

Yang, J.H., Yang, J.-I., Kim, H.-J., Chun, D.H., Lee, H.-T., Jung, H., 2010. Two regime transitions to pseudo-homogeneous and heterogeneous bubble flow for various liquid viscosities. Chem. Eng. Process.: Process Intensif. 49, 1044-1050.

Yoshida, F., Akita, K., 1965. Performance of gas bubble columns: volumetric liquid-phase mass transfer coefficient and gas holdup. AIChE J. 11, 9-13.

Zahradnik, J., Fialova, M., Ruzicka, M., Drahoš, J., Kavstanek, F., Thomas, N.H., 1997. Duality of the gas-liquid flow regimes in bubble column reactors. Chem. Eng. Sci. $52,3811-3826$.

Zehner, P., Kraume, M., 2005. Bubble Columns, Ullmann's Encyclopedia of Industrial Chemistry. Wiley-VCH Verlag GmbH \& Co. KGaA, Weinheim.

Ziegenhein, T., 2016. Fluid dynamics of bubbly flows (Ph.D Thesis). Technische Universität Berlin.

Ziegenhein, T., Rzehak, R., Lucas, D., 2015. Transient simulation for large scale flow in bubble columns. Chem. Eng. Sci. 122, 1-13.

Zuber, N., Findlay, J.A., 1965. Average volumetric concentration in two-phase flow systems. J. Heat. Transf. 87, 453-468. 